\title{
2009 UK/US Nuclear Engineering Workshop Report
}

April 2009

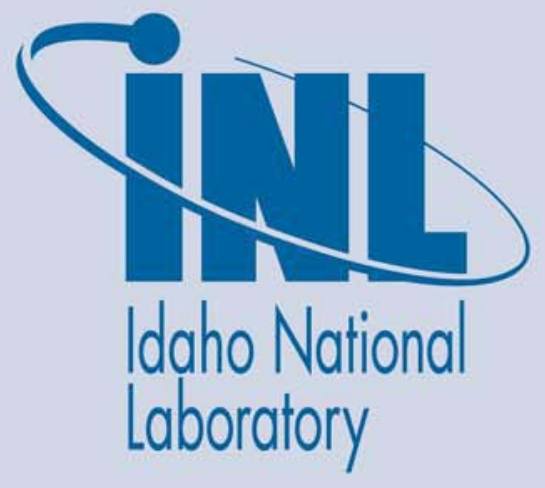

The INL is a U.S. Department of Energy National Laboratory operated by Battelle Energy Alliance 
INL/EXT-09-16286

\section{UK/US Nuclear Engineering Workshop Report}

April 2009

Idaho National Laboratory
Idaho Falls, Idaho 83415

http://www.inl.gov

Prepared for the

U.S. Department of Energy

Office of Nuclear Energy

Under DOE Idaho Operations Office

Contract DE-AC07-05ID14517 


\section{UK/US Nuclear Engineering Workshop Report}

\section{April 20-21, 2009 Washington D.C.}

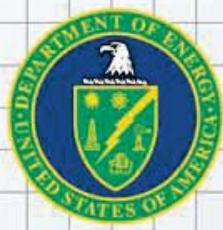

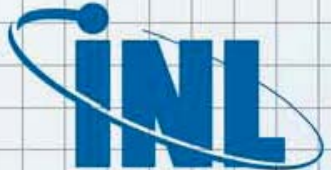

Idaho National Laboratory

\section{EPSRC}

Engineering and Physical Sciences Research Council 


\section{DISCLAIMER}

This information was prepared as an account of work sponsored by an agency of the U.S. Government. Neither the U.S. Government nor any agency thereof, nor any of their employees, makes any warranty, expressed or implied, or assumes any legal liability or responsibility for the accuracy, completeness, or usefulness, of any information, apparatus, product, or process disclosed, or represents that its use would not infringe privately owned rights. References herein to any specific commercial product, process, or service by trade name, trade mark, manufacturer, or otherwise, does not necessarily constitute or imply its endorsement, recommendation, or favoring by the U.S. Government or any agency thereof. The views and opinions of authors expressed herein do not necessarily state or reflect those of the U.S. Government or any agency thereof. 


\section{Executive Summary}

This report summarizes the 2009 UK/US Nuclear Engineering Workshop held April 20-21 in Washington D.C. to discuss opportunities for nuclear engineering collaboration between researchers in the United States and the United Kingdom.

The workshop identified five key driving requirements for collaboration:

- Extending the life and ensuring the health of the fleet of current reactors

- Stabilizing the current fuel inventory

- Bringing GEN-III+ plants online

- Bringing advanced reactors online

- Closing the fuel cycle.

Attendees from national laboratories and universities in both countries discussed potential areas for collaboration and identified six engineering categories that guided the structure of the workshop:

- Fuel Technology

- Reactor Materials Performance

- Reactor Design, Operations, and Monitoring

- Spent Nuclear Fuel and Waste Management and/or Post Operation

- Decontamination and Decommissioning

- Safety, Security, and Safeguards.

Smaller groups consisting of researchers from each of these categories discussed specific areas for collaboration, including the needs, capabilities, and opportunities in each nation that could benefit from collaborative research efforts. Areas for potential collaboration ranged from modeling and simulation projects to instrumentation and control system design projects.

The main goal of the workshop was to foster individual collaborations between British and American researchers from each group and that these would develop on a case-by-case basis. In several months, the chairs will contact participants to assess the workshop's effectiveness in facilitating collaboration. 


\section{ACKNOWLEDGEMENTS}

The 2009 U.K./U.S. Nuclear Engineering Workshop was organized by the U.K. Engineering and Physical Sciences Research Council and Idaho National Laboratory (INL). Special thanks are extended to the workshop organizers:

- Dr. Steve Elsby, U.K. Research Councils Energy Programme, Workshop Chair

- Richard Rankin, INL, Workshop Co-Chair

- Naomi Webber, U.K. Research Councils, U.S. Office.

In addition, the workshop organizers thank the staff of the British Embassy in Washington, D.C., for providing support for the workshop, INL facilitators Lori Braase and Bryan Parker, and the American Geophysical Union for hosting the event.

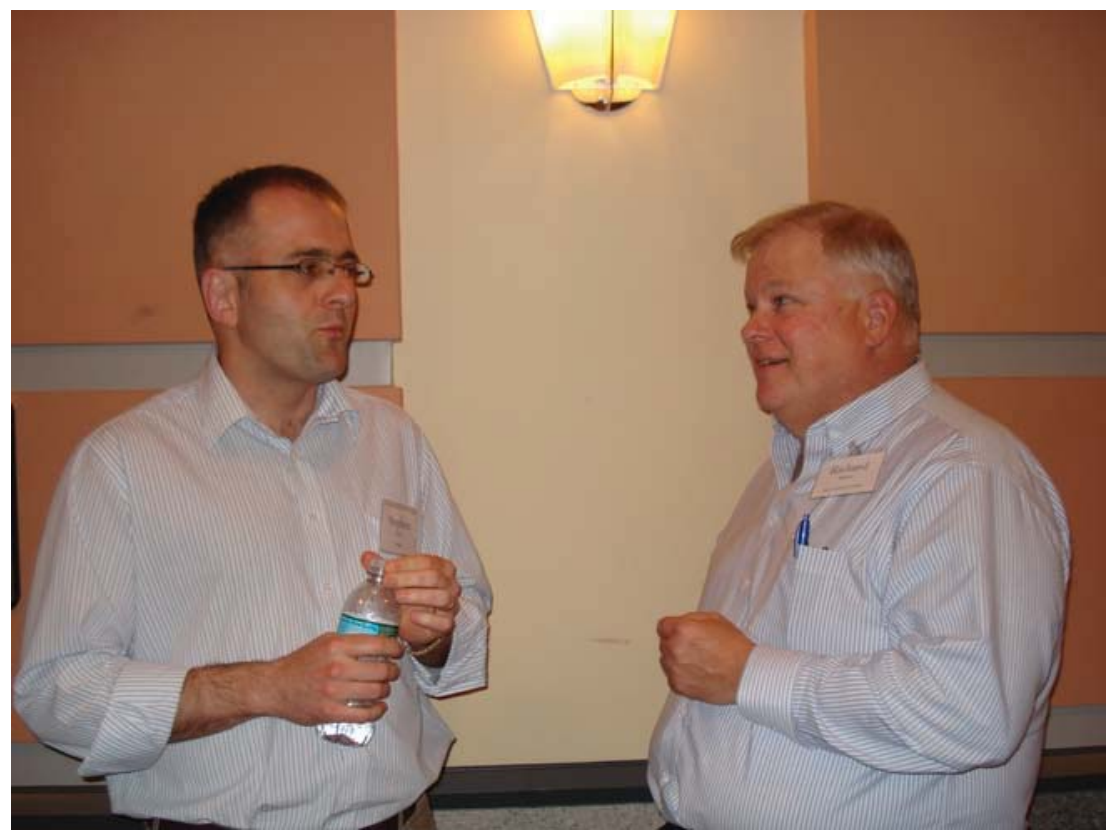




\section{CONTENTS}

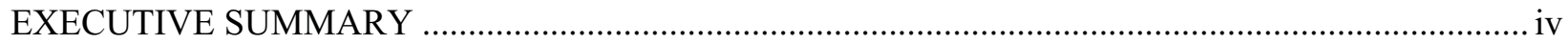

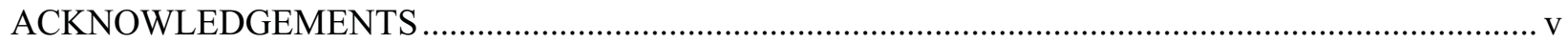

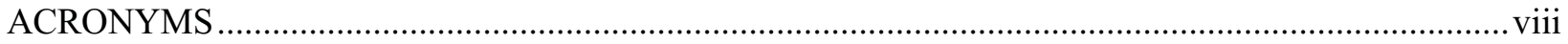

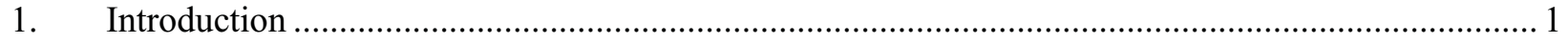

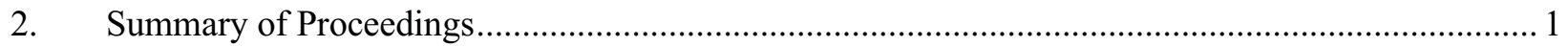

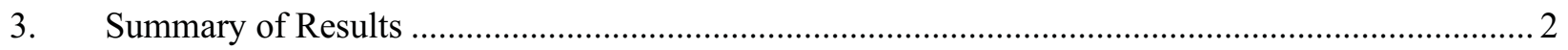

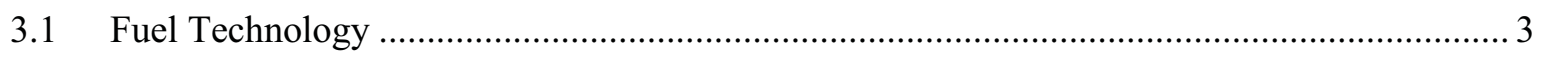

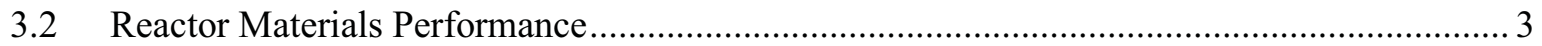

3.3 Reactor Design, Operations, Monitoring, and Safety ...................................................... 4

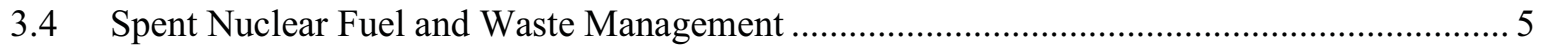

3.5 Decommissioning and Decontamination .................................................................... 7

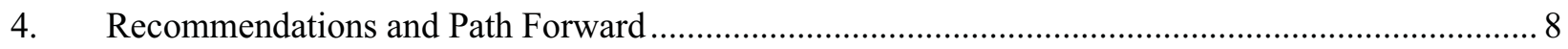

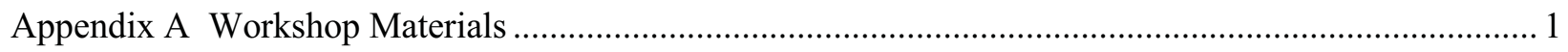

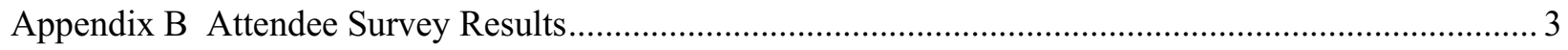

\section{Figures}

Figure 1. Extending the life and health of current reactors is a

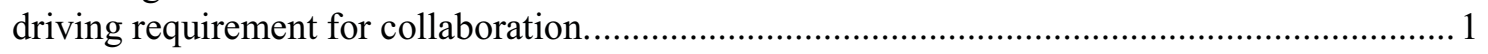

Figure 2. Workshop participants identified five areas for collaboration................................................. 2

Figure 3. Fuel fabrication and performance are important areas for potential collaboration....................... 3

Figure 4. The next generation of reactors will require materials that can withstand high

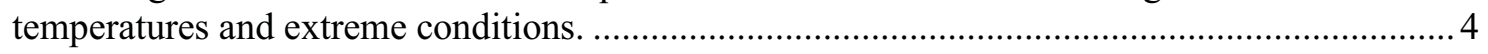

Figure 5. Reactor design, monitoring, operations, and safety are also potential areas for

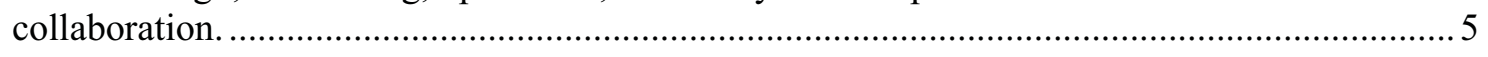

Figure 6. Participants worked together to identify potential areas for collaboration................................ 6

Figure 7. Working groups created important opportunities for networking and discussion....................... 7 


\section{ACRONYMS}

$\begin{array}{ll}\text { ATR } & \text { Advanced Test Reactor } \\ \text { CMSN } & \text { Computational Materials Science Network } \\ \text { D\&D } & \text { Decommissioning and Decontamination } \\ \text { GEN-III+ } & \text { Generation Three Plus (nuclear plants) } \\ \text { I\&C } & \text { instrumentation and controls } \\ \text { INL } & \text { Idaho National Laboratory } \\ \text { NDA } & \text { Nuclear Decommissioning Authority (U.K.) } \\ \text { NEA } & \text { Nuclear Energy Agency } \\ \text { NNL } & \text { National Nuclear Laboratory (U.K.) } \\ \text { PWSCC } & \text { primary water stress corrosion cracking } \\ \text { UK } & \text { United Kingdom } \\ \text { US } & \text { United States }\end{array}$




\section{UK/US Nuclear Engineering Workshop Report}

\section{Introduction}

On April 20 and 21, 2009, researchers and policymakers from the United Kingdom (UK) and the United States (US) met in Washington, D.C., to discuss nuclear engineering capabilities, needs, and potential avenues for collaboration between the two nations.

The workshop identified the following five key driving requirements for nuclear engineering research and collaboration between the U.K. and the U.S.:

- Extending the life and ensuring the health of the fleet of current reactors

- Stabilizing the current fuel inventory

- Bringing GEN-III+ plants online

- Bringing advanced reactors online

- Closing the fuel cycle.

The workshop was designed to stimulate collaboration that will meet these driving requirements.

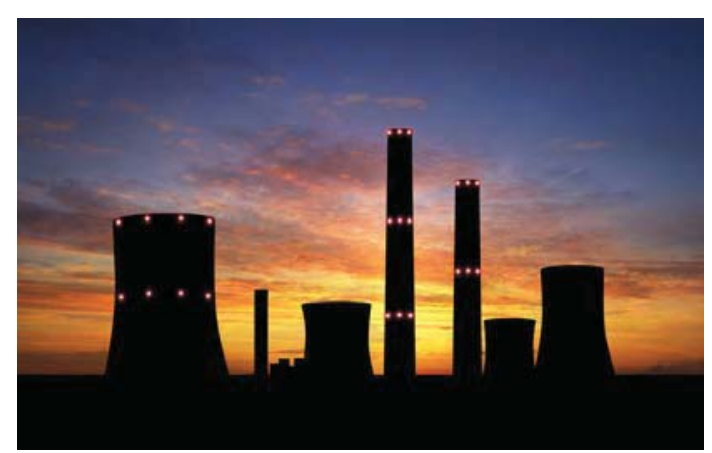

Figure 1. Extending the life and health of current reactors is a driving requirement for collaboration. This report summarizes the proceedings of the two-day workshop, lists the results of these proceedings in six technical areas, and presents a recommended path forward for future collaborations.

\section{Summary of Proceedings}

The conference convened with opening remarks from Steve Elsby, Research Councils Energy Programme (UK) and Dave Hill, Idaho National Laboratory, (US). During introductions, attendees were encouraged to list the individuals that may provide opportunities for future collaborations. They were then given time to network with those identified on their lists. A brainstorming session followed to discuss and identify the categories of nuclear engineering research that would guide the structure of the workshop. The group identified the following categories:

- Fuel Technology

- Reactor Materials Performance

- Reactor Design, Operations, and Monitoring

- Spent Nuclear Fuel and Waste Management and/or Post Operation

- Decontamination and Decommissioning

- Safety, Security, and Safeguards.

The attendees also identified several cross-cutting areas and issues that affect all six of categories in the previous list. Collaborations in these areas can assist all of nuclear engineering. The cross-cutting activities are:

- Life Extension \& Licensing

- Instrument and Controls 
- Economic Issues including Comparative Energy Study (other energy sources)

- Materials Development and Testing

- Modeling and Simulation (Cross-cutting)

- Public Understanding of Risk

- Skills, Training, and Education

- Mechanistic Understanding

- Regulatory Frameworks and Support of Safety Case Development.

Following the identification of research categories and cross-cutting areas, workshop facilitators Bryan Parker and Lori Braase provided instructions for a "gallery walk," a group technique to encourage individual information gathering and ad hoc small-group discussion. The exercise focused on the nuclear engineering research categories, and the result was a list of gaps and needs for each nation and a list of attendees interested in collaboration (see Appendix A).

Following the gallery walk, groups were formed for each research category to focus on specific research capabilities, needs, and opportunities for collaboration. The workshop was then adjourned until Tuesday morning.

Tuesday morning sessions continued the research discussions for each collaboration group. These sessions included prioritizing key collaborations and filling in "action" templates to document opportunities, needs, and areas of

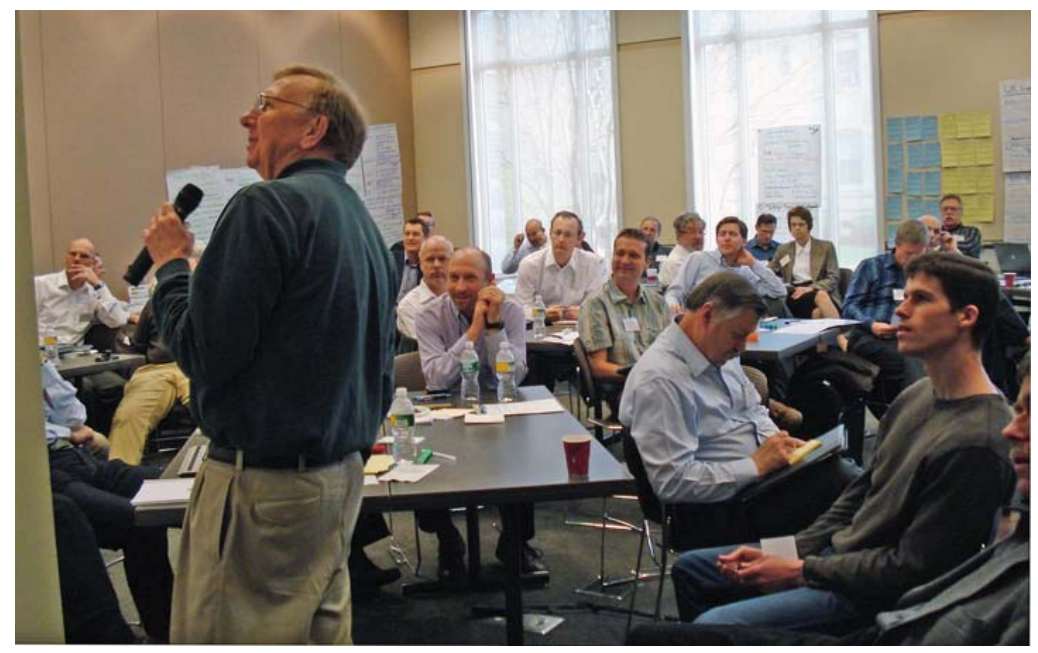

Figure 2. Workshop participants identified five areas for collaboration. strength. The contents of these templates are summarized in Section 3 and presented in more detail in Appendix A.

Following lunch, Paul Howarth, National Nuclear Laboratory (NNL, UK), discussed market opportunities in the UK. Finally, each collaboration group prepared and presented the key collaborations it developed.

Steve Elsby closed the workshop with a discussion of the path forward for collaborations between the two nations.

\section{Summary of Results}

This section summarizes the results of the meetings between the collaboration groups, which were organized according to the categories for nuclear engineering research that were identified by the workshop attendees on Monday morning. 


\subsection{Fuel Technology}

The Fuel Technology area consisted of four groups in which attendees identified capabilities, areas of need, and steps towards collaboration. These subgroups included:

- Modeling and Simulation of Fuel Fabrication

- Experimental Verification of Fuel Fabrication

- Modeling and Simulation of Fuel Performance

- Experimental Verification of Fuel Performance.

The Modeling and Simulation of Fuel Fabrication group determined that an opportunity exists in this area to use modeling to explore well-defined $n$-dimensional variable space to find critical combinations of variables. Multiscale, multiphysics models can be used to address or predict particle morphologies and flow and packing characteristics. Needs include development of non-ceramic fuels and assistance with sintering models. The next step is to identify a funding stream.

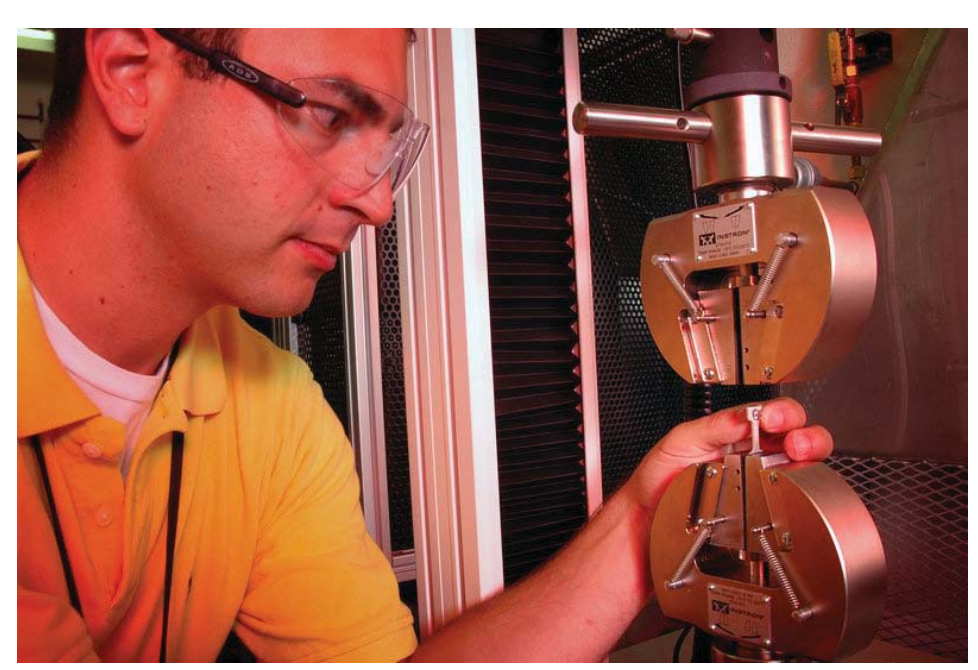

Figure 3. Fuel fabrication and performance are important areas for potential collaboration.
Opportunities for collaboration in Experimental Verification of Fuel Fabrication include validating morphology changes, validating process models, and testing predictions of porosity and mechanical properties as well as other fuel fabrication properties.

In the area of Modeling and Simulation of Fuel Performance, the group identified extensive programs currently underway that A US/UK collaboration could contribute to by coordinating and raising awareness of these programs. The next steps in this collaboration include: (1) developing and maintaining an email list, (2) attending meetings scheduled on May 20 between Robin Grimes and Jim Tulenko to continue forming collaborations, and (3) using the Computational Materials Science Network (CMSN) and the Nuclear Energy Agency (NEA) to coordinate with other entities.

The Experimental Verification of Fuel Performance group identified Idaho National Laboratory's (INL's) Advanced Test Reactor (ATR) fuel performance experiments as a potential opportunity for collaboration. The next steps are to share data from previous experimental results and formulate new experiments that meet the needs of both nations.

\subsection{Reactor Materials Performance}

The Reactor Materials Performance area was also split into four subgroups:

- Irradiation Embrittlement

- Materials Library

- High-Temperature Structural Materials 
- Primary Water Stress Corrosion Cracking (PWSCC) and Irradiation.

Each of these groups assessed opportunities, needs, and next steps.

The Irradiation Embrittlement group identified three potential opportunities for collaboration: (1) life extension, (2) provision of U.S. facilities, and (3) expansion of multiscale modeling basic physics. The needs identified by this group include defining the scope and money. The group coordinators agreed to define a working group as the next step towards collaboration.

The Materials Library group determined that combining the US/UK knowledge to create a database of materials and characterization properties was their prime opportunity for collaboration. The users of such a database will likely come from the fuels development and waste processing projects. As such, the group determined that the next step would be to involve members of the Fuel Technology area and the Spent Nuclear Fuel and Waste Management area.

The group assessing

High-Temperature Structural

Materials discussed several collaborative opportunities: (1) overlaps with fusion; (2) potential access to facilities, including irradiation; (3) fabrication processes; (4) life extension; and (5) potential for links with conventional power industry's interest in high-temperature materials. As they move forward, this group will (1) define the scope in terms of understanding the combined effects of stress, temperature, and radiation on structural materials; (2) pinpoint other needs; and (3) define funding. The group coordinators determined that the next step would be to define a working group.

Finally, the PWSCC and Irradiation group examined the possibilities that US facilities might have for irradiation and post testing analysis impact of cold work, mechanistic understanding, etc. The group agreed that their primary needs include defining scope, needs, and funding. The next steps involve collaboration with the Waste Management area and the group coordinators defining a working group.

\subsection{Reactor Design, Operations, Monitoring, and Safety}

The Reactor Design, Operations, Monitoring, and Safety area was divided into five subgroups. These subgroups also identified capabilities, areas of need, and steps towards collaboration. They included:

- Uncertainty Quantification

- Nuclear Data Generation

- Instrumentation and Controls (I\&C) 


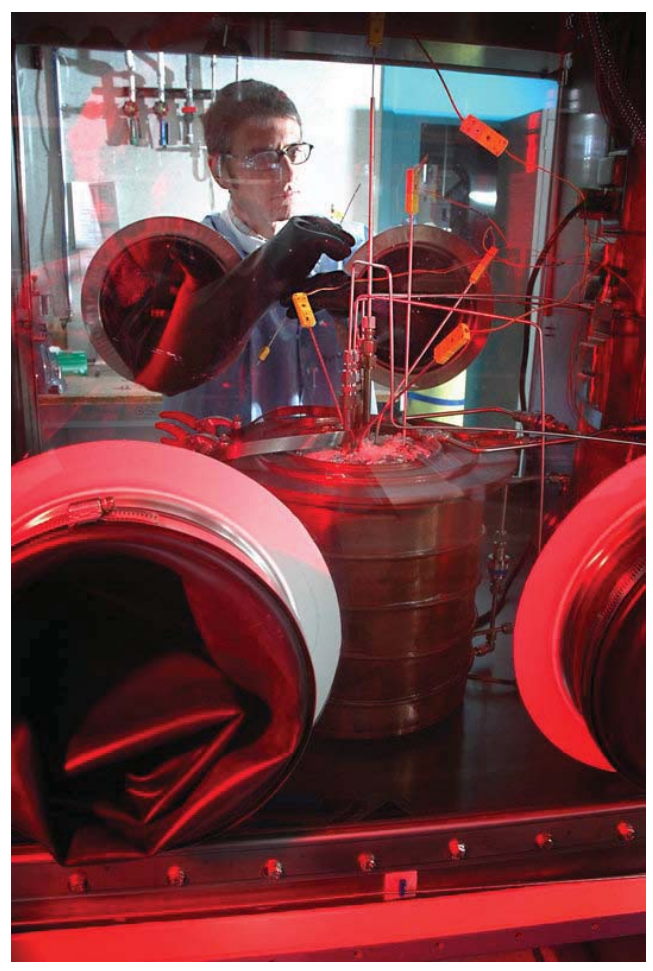

Figure 5. Reactor design, monitoring, operations, and safety are also potential areas for collaboration.

student education and training.
- Fundamentally based Modeling for Severe Accidents

- Self-adapted Modeling.

The group tasked to consider Uncertainty Quantification focused on issues of highly nonlinear systems, including data assimilation and experiment design. This group also focused on aging phenomena. They identified a specific need to understand the impact of uncertainty on lifetime prediction for reactors. This area links back to the Reactor Materials issues discussed in Section 3.2.

The Nuclear Data Generation group identified an opportunity for collaborating on the generation of nuclear data via differential experiments and nuclear models.

The I\&C group discussed issues associated with operation of Gen IV reactors and identified several UK resources for statistical software analysis and testing.

The group tasked with identifying areas for collaboration in Fundamentally based Modeling for Severe Accidents determined that links can be made between fundamental modeling and education and training using advanced reactor simulators. The group hopes to make these advanced simulators available for

The Self-adapted Modeling group focused on goal-oriented approaches to modeling that use algorous multiscale closure relations.

\subsection{Spent Nuclear Fuel and Waste Management}

Spent Nuclear Fuel and Waste Management area attendees split into five subgroups as well:

- Waste System Performance Assessment Modeling

- Understanding Processes in Waste Forms

- Irradiated Materials Performance

- Sample Harvesting

- Advanced Partitioning and Separations.

Each of these groups assessed opportunities, needs, and next steps.

The Waste System Performance Assessment Modeling group discussed several potential opportunities by asking the following questions as they relate to the integration of coupled processes that exhibit complex behavior at a wide range of scales:

- How can multiscale models be applied to engineered and natural barrier systems? 
- How do we model the interactions between radionuclides, mineralogy, and microbial populations in a thermal-hydro-mechanical framework?

- How can we bound and reduce critical sources of uncertainty in prediction of waste system performance?

The group also identified the need to contact key individuals that can bring expertise from other fields (hydrocarbon, $\mathrm{CO}_{2}$ sequestration geo-microbiology, rock structure, chemistry, etc.) to this area. Group members felt the next step in this process would be to hold a 1-2 day workshop to build collaborative U.S./U.K. research teams to address the proposed research questions above.

The Understanding Processes in Waste Forms group discussed eight potential areas of opportunity:

1. Structural and thermodynamic characterization and kinetics

2. Model relevant experimental conditions

3. Bridging scales of model

4. Interfacial phenomena

5. Separating thermal and radiation effects

6. Identification of specific waste forms

7. Taking advantage of advances in computing

8. Multiple characterization techniques.

The group agreed that the identification of specific systems to study, the exchange of samples and people, and logistics were the biggest areas of need. The group also agreed that the best next step forward is to hold a special session on this topic at the MRS 2010 Spring Meeting.

\section{Members of the Irradiated Materials}

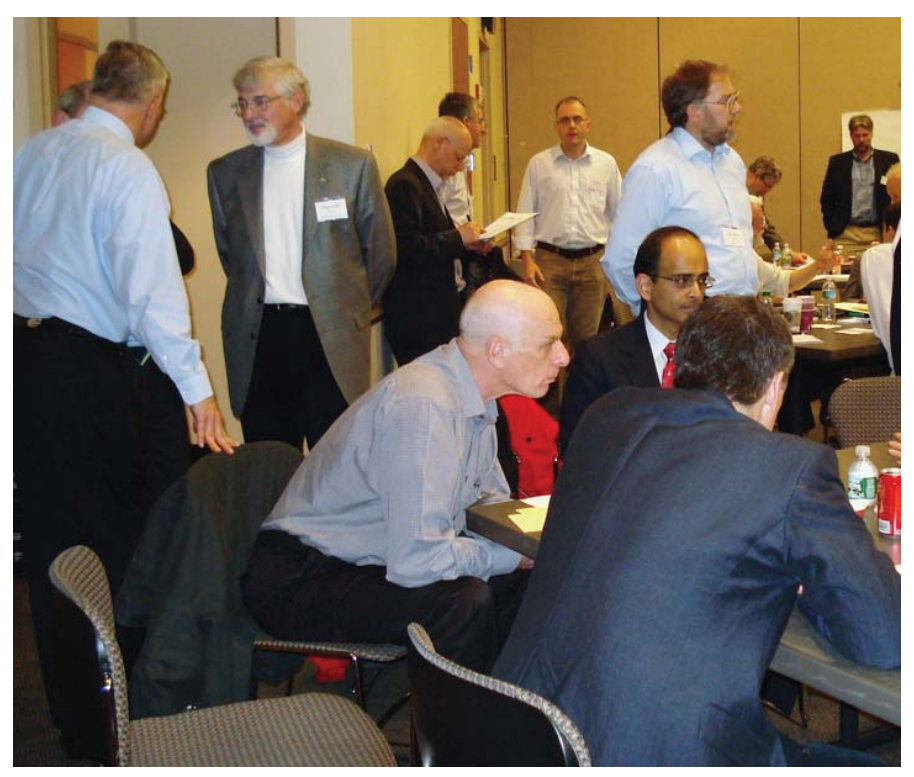

Figure 6. Participants worked together to identify potential areas for collaboration.

Performance group identified two collaborative opportunities regarding complimentary expertise at the University of Michigan: (1) coordination of future programs, and (2) comparisons of lessons learned on fuels, oxides, metals, nitrides, oxides, structural materials, metals, and graphite. The group felt that defining both a matrix of expertise and a mechanism for the exchange of personnel were their largest needs. The next step forward would be to build linked research programs and collaborate with the Understanding Processes in Waste Forms group.

The Sample Harvesting group agreed that their best opportunities for collaboration included these three items: (1) gaining reciprocal access to inventions of well-characterized samples, (2) exploiting pooled characterization tools, and (3) generating new data to link to predictive capabilities. The group decided that several needs exist, including the need to organize a meeting and linking activity with National Nuclear Laboratory (U.K.), INL (U.S.), Argonne National Laboratory (U.S.), and Oak Ridge National Laboratory (U.S.) to define the path forward, because this effort requires buy in from all national regulators and industries. In addition, they feel that the next step will be to coordinate harvesting with the Understanding Processes in Waste Forms group and come up with an action plan that addresses the following:

- Construct matrix of interests, participants, and links 
- Develop strategy and research priorities

- Present coordinated objectives and routes to success

- Utilize synergies to optimize program

- Seek seed funding for workshop and exchange visits

- Leverage progress - feasibility

- Establish a coordinated and responsive research program.

Finally, the Advanced Partitioning and Separations group identified four areas as their greatest opportunities for collaboration: (1) simplifying processing schemes, (2) improving radiation stability of materials, (3) providing Gen IV support, and (4) dealing with spent fuel/orphan waste disposition. Needs include:

- Understanding of the underpinning and the chemistry of existing schemes

- Development of new, simplified schemes

- Development of new schemes to improve Rad Stability

- Mechanics.

The group also identified the following as next steps:

- Discussions between National Laboratory and academic partners in both US/UK

- NNL/NDA discussions in U.K.

- Mechanism and action plan detail.

\subsection{Decommissioning and Decontamination}

The Decommissioning and Decontamination (D\&D) area attendees broke into two subgroups: (1) Measurement for D\&D, and (2) Knowledge \& Technology Transfer. As with the other areas and groups, these subgroups were tasked with identifying capabilities, areas of need, and steps towards collaboration.

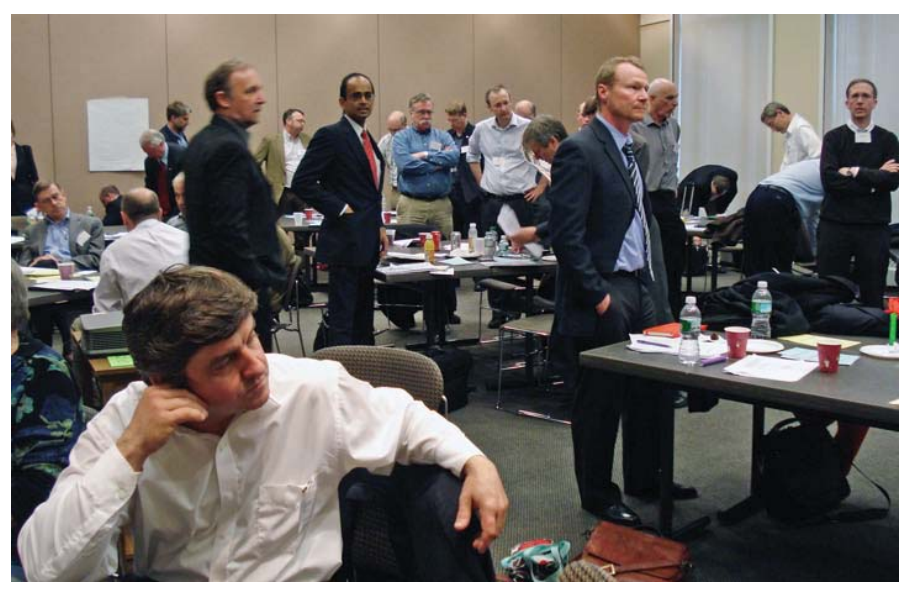

Figure 7. Working groups created important opportunities for networking and discussion.
The Decommissioning and Decontamination group members agreed that their best opportunities include working better, faster, cheaper, and safer, and providing better analyses, especially on nonpenetration, than in the past. Needs are to focus on utilizing measurement, signal processing, and deployment. Next steps are to further develop ideas and identify a specific target application as soon as possible.

\section{The Knowledge \& Technology} Transfer group members felt that their best opportunities would be improving D\&D techniques, waste characterization, and volume reduction, as well as to better 
understand problems and provide fresh insight. The group also decided to hold follow-on workshops.

\section{Recommendations and Path Forward}

It was generally felt that this workshop, and others like it, served as a worthwhile means to generating collaboration between the technical communities of the U.K. and the U.S. Several interactions following the workshop have resulted stronger international teams that can better address challenges in the nuclear field. Examples of these are:

A. UK and US organizations have entered into strategic agreements.

- INL and the University of Oxford have signed a memorandum of understanding to cooperate in analyzing the radiation performance of reactor materials in support of the Reactor Materials Performance topical area

B. Informal or formal collaboration between UK-US organizations have developed.

- The University of Birmingham, the University of Warwick, and INL have prepared a joint proposal to address advanced separations technology for the D\&D topical area.

- The University of Birmingham and INL are working together on advanced materials to support a joint proposal to address the Reactor Materials Performance topical area.

- Cambridge University and Pacific Northwest National Laboratory have collaborated on comparing radiation effects in Zircon and Xenotime supporting the Spent Nuclear Fuel and Waste Management topical area.

- Imperial College; Manchester, Lancaster, and Reading Universities; the NNL; and INL have begun work on a research project (EPSRC MBAse consortium, start date April 1, 2010) that will include secondments of postdocs and students to US facilities in support of the Spent Nuclear Fuel and Waste Management topical area.

- Manchester and INL have conducted seminars and student exchanges initially in support of the Spent Nuclear Fuel and Waste Management topical area, with potential extension to all other areas.

- The University of Illinois at Urbana-Champaign and the University of Salford have begun discussing a collaboration to study various properties of graphite in support of the Reactor Materials Performance topical area.

- Imperial College and Los Alamos National Laboratory have been engaged in an informal collaboration in support of the Fuel Technology and Spent Nuclear Fuel and Waste Management topical areas. This has resulted in a successful grant application, joint participation in professional society meetings, and publications.

- Imperial College and the University of California at Berkeley are engaged in collaborative discussions in support of the Reactor Materials Performance topical area and have recently been successful on a proposal.

- University of Florida and Manchester University are collaborating in modeling of nuclear fuels in support of the Fuel Technology topical area 
- The University of Florida, Imperial College, and Oak Ridge National Laboratory are working together on a joint Department of Energy proposal for modeling of nuclear fuels in support of the Fuel Technology topical area.

C. US National Laboratories hosted UK interns for developmental training and experience.

- INL hosted a UK NDA employee in civil structural engineering in the Decommissioning and Decontamination topical area

- The University of Bristol together with their spin-out company VEQTER Ltd are providing a residual stress workshop as part of the EPRI-MRP conference in July 2010 at Colorado Springs in support of the reactor design, operations, monitoring and safety topical area.

D. Recommendations from the workshop are:

- That the UK and the US develop more formal support mechanisms for research interactions between the UK Research Councils and the US Department of Energy. The output of this workshop forms a good starting point.

- That the UK and the US explore an exchange mechanism based around the alignment of specific existing UK/US research funding with a small amount dedicated to supporting and enabling research exchanges. 
Appendix A

Workshop Materials 



\section{UK/US NUCLEAR ENGINEERING WORKSHOP \\ American Geophysical Union (AGU) \\ Washington DC \\ April 20- 21, 2009}

Meeting Objective: Focus on opportunities in areas where UK/US collaborations make sense and fill the needs for one or both countries.

Meeting Outcome: Develop a list of UK/US collaborations in specific areas of research, including the next steps and points-of-contact for each country.

\section{AGENDA}

Monday, April 20, 2009

8:00 Refreshment

8:30 Welcome/Opening Remarks

Steve Elsby, UK

Dave Hill, US

9:00 Introductions

All

10:20 Networking (15 minute break included)

All

11:20 Brainstorm, Discuss, and Gain Consensus on

All

5-7 Categories/Areas of Nuclear Engineering Research

All

12:00 Networking Lunch

All

13:00 Gallery Walk to Identify Research Gaps / Needs

Facilitators

14:00 Gallery Walk Review and Clarification by Category

14:45 Break

15:15 Collaboration Identification (to fill the previously

All identified gaps or needs)

16:00 Form Collaboration Groups by Category of Interest and

All begin research discussions

17:00 Adjourn 


\section{UK/US NUCLEAR ENGINEERING WORKSHOP \\ American Geophysical Union (AGU) \\ Washington DC \\ April 20- 21, 2009}

\section{AGENDA}

Tuesday, April 21, 2009

8:00 Refreshment

8:30 Welcome / Review / Process for Day Two

Steve Elsby \& Facilitators

8:45 Continue Research Discussions to Identify Key

Collaboration Collaborations

Groups

10:00 Break

10:30 Prioritize Collaborations and Fill in Action Plan Templates

Collaboration Groups

12:00 Networking Lunch

13:00 UK Market Opportunities Presentation

Paul Howarth

14:00 Prepare Presentation of Key Collaborations

(by Main Areas)

Collaboration

Groups

15:00 Break

15:15 Present Key Collaborations to Entire Group

Collaboration

Groups

16:30 Path Forward (Actions and Success Strategy)

Steve Elsby

17:00 Adjourn 


\section{UK/US NUCLEAR ENGINEERING WORKSHOP \\ American Geophysical Union (AGU) \\ Washington DC \\ April 20- 21, 2009}

\section{Nuclear Engineering Categories}

1. Fuel Technology
a. Increase thermal conductivity $\left(\mathrm{MOX} \& \cup_{2}\right)$
b. Improve cladding materials and performance (LOCA issues)
c. Fuel Performance Code
d. Development of alternative materials to improve efficiency and burnup
e. Advanced Fuel Cycles
f. Advanced and Alternative Fuels (materials \& designs)
g. Fuel fabrication

2. Reactor Materials Performance
a. Analysis and Safety
b. Gen IV (including VHTR)
c. Life extension, lifetime prediction
d. Advanced LWR systems
e. Construction Engineering
f. Materials \&Technologies, such as pressure vessels, cooling systems, etc.
g. Corrosion and Erosion

3. Reactor Design, Operations, and Monitoring
a. Controls
b. Safety, safeguards, security
c. Radiation Detection and Measurement
d. Water Chemistry, including the scum

4. SNF \& Waste Management and/or Post Operation
a. Reprocessing
b. Waste forms
c. Permanent Storage
d. Disposal
e. Transmutation of waste (Reduction of volumes and waste recycling)
f. Environmental Waste Management \& Legacy Waste
g. Advanced Fuel Cycles
h. Materials Harvesting-International Collaboration
i. Extension of Lifetime of existing plants
ii. Initial specs of new reactors ( 60 years)
iii. Material exposure - many years of service
iv. Documentation
v. Need to identify best characterized plants
vi. Share \& characterize rescued samples

5. Decontamination \& Decommissioning

a. Legacy waste management

b. Reduction of volumes \& waste recycling 


\section{UK/US NUCLEAR ENGINEERING WORKSHOP \\ American Geophysical Union (AGU) \\ Washington DC \\ April 20- 21, 2009}

6. Safety (radiological/environmental) \& Security \& Safeguards
a. Proliferation Resistance
b. Monitoring
c. International activities
d. Public and government confidence
e. Risk analysis
f. Advanced Safety Systems

\section{Cross Cutting Activities}

- Life Extension \& Licensing

- Instrument and Controls

- Economic Issues including Comparative Energy Study (other energy sources)

- Materials Development \& Testing

- Modeling \& Simulation (Cross-cutting)

- Tied with Experiments \& Testing

- Multiphysics and Multiscale Modeling

- Public understanding of risk

- Closing the fuel cycle

- General energy sources

- Skills, Training and Education

- Mechanistic Understanding

- Uncertainty Propagation

- Experimental data

- Regulatory Frameworks and Support of Safety Case Development

\section{Key Drivers: Imperatives (support the categories)}

- Extend the life and ensure health of fleet

- Stabilize the current fuel inventory

- Bring GEN-III+ plants online

- Bring Advanced reactors online

- Small advanced LWR

- HTR

- Burner Reactors

- Close the fuel cycle 


\section{UK/US NUCLEAR ENGINEERING WORKSHOP \\ American Geophysical Union (AGU) \\ Washington DC \\ April 20- 21, 2009}

\section{Gallery Walk by Category}

\subsection{Fuel Technology}

\begin{tabular}{|ll}
\hline \multicolumn{1}{|c}{ US Gaps/ Needs } \\
\hline 1. & $\begin{array}{l}\text { Link atomistic details to } \\
\text { phenomena at larger scales. }\end{array}$ \\
\end{tabular}

2. Fission product transport (SiC, ZrC, etc.).

3. Radiation damage of ceramics (Sic, IMF etc.).

4. PIE.

5. Samples for thermochemical studies of fuels, novelty, etc.

6. Fundamental understanding of fuel processing parameters effect on final fuel characterization.

7. Modeling \& Simulation of heat transfer.

8. Multiphysics modeling.

9. Thoria fuel cycle.

10. Database for validation of fuel models and codes.

11. Advanced fuel performance models and codes (MOX, UO2, UC, UN, U-Mo)

12. Probabilistic fuel performance models.

13. Crud modeling and prediction related to fuel performance.

14. Development of novel nuclear fuel for advanced burners and LEU fuel for research reactors.

15. Fuel/Clad interactions.

\begin{tabular}{|c|c|}
\hline UK Gaps/Needs & $\begin{array}{l}\text { Interested in } \\
\text { Collaboration }\end{array}$ \\
\hline $\begin{array}{l}\text { 1. Radiation induced degradation/damage } \\
\text { of fuel forms. } \\
\text { 2. Facility for test fuel fabrication (MOX, } \\
\text { HTR Fuel). } \\
\text { 3. Access to test fuel irradiations. } \\
\text { 4. PIE Facilities. } \\
\text { 5. Mechanistic understanding of high } \\
\text { burnup (HBU) fuels. } \\
\text { 6. Deep burn/disposition of Pu. } \\
\text { 7. } \text { Modeling and measuring thermal } \\
\text { conductivity for IMFs. } \\
\text { 8. Powder mixing. } \\
\text { 9. Helium behavior. } \\
\text { 10. Experimental verification of fission } \\
\text { product behavior in fuel (e.g., joint } \\
\text { experiment/modeling work with same } \\
\text { aims. } \\
\text { 11. Thermodynamic modeling of phased } \\
\text { development in fuels. } \\
\text { 12. Advanced fuel manufacture \& testing. } \\
\text { 13. Fuel cladding performance in reactive } \\
\text { environments. } \\
\text { 14. In pile fuel performance. } \\
\text { 15. Rod bundle test facility. } \\
\text { 16. Carbide/nitride/oxide ceramic fuel } \\
\text { assessment. } \\
\text { 17. High burnup studies for Gen III. } \\
\text { 18. HTR Fuel Test manufacture facility } \\
\text { 19. Common fuel performance codes. } \\
\text { 20. Manufacture and in-core assessment } \\
\text { through experiments and M\&S of dirty } \\
\text { fuel. }\end{array}$ & $\begin{array}{l}\text { Ram Devanathan } \\
\text { Jim Tulenko } \\
\text { Todd Allen } \\
\text { Sudarshan Loyalka } \\
\text { Simon Pinblott } \\
\text { Randy Nanstad } \\
\text { Bill Martin } \\
\text { lan Farnan } \\
\text { Francis Livens } \\
\text { John Ireland } \\
\text { Alex Navrotsky } \\
\text { Brian Wirth } \\
\text { lan Hutchinson } \\
\text { Jack Brenizer } \\
\text { Totju Totev } \\
\text { Igor Bodnar } \\
\text { Andy Klein } \\
\text { Ping Xiao } \\
\text { Roger Smith } \\
\text { Simon Biggs } \\
\text { Robin Grimes } \\
\text { Neil Hyatt } \\
\text { Brent Heuser } \\
\text { Richard Rankin } \\
\text { Jason Hayward } \\
\text { Chris Grovenor } \\
\text { Paul Turinsky } \\
\text { (contact for K.L. } \\
\text { Murty/Jacob Eapen) }\end{array}$ \\
\hline
\end{tabular}




\section{UK/US NUCLEAR ENGINEERING WORKSHOP \\ American Geophysical Union (AGU) \\ Washington DC \\ April 20- 21, 2009}

\subsection{Reactor Materials Performance}

\section{US Gaps/ Needs}

1. Radiation damage thermodynamic studies, samples.

2. Develop better interatomic potentials for alloys.

3. High temperature components and structure.

4. LWR crack initiation and growth.

5. Kinetic models of microstructural evolution.

6. Corrosion (CO2, sodium, molten salt)

7. Predictive, physically-based models of component/materials lifetimes.

8. Irradiation testing.

9. Radiation-resistant structural materials.

10. UQ prediction of macro properties from multiphysics / multiscale modeling.

11. Characterization of the atomicscale structure and composition of nanometer scale $\mathrm{Y}$-Ti-O features in advanced oxide dispersion strengthened steels.

\begin{tabular}{|ll} 
& \multicolumn{1}{c}{ UK Gaps/Needs } \\
1. & $\begin{array}{l}\text { Understanding of interfacial } \\
\text { properties/performance of materials. } \\
\text { Good models of multi-component } \\
\text { systems - steels - FeCr alloys etc., from } \\
\text { classical atomistic viewpoint (potentials) } \\
\text { to structural scales. }\end{array}$
\end{tabular}

3. Better understanding of stress corrosion cracking.

4. Material properties after long-term aging.

5. Complementary high temperature materials test facilities.

6. Access to facilities to irradiate and test samples in reactor environments including PWR \& AGR.

a.Ferritic, austenitic, nickel-based, zirconium, graphite, polymers

b. ODS steels, SiC-SiC, martensitic.

7. Self explaining long-time scale algorithms for long-time matured property prediction (bridging time scales).

8. How do we fabricate / weld ODS steels?

9. Better understanding of combined efforts of stress \& temperature \& irradiation \& fatigue $\&$ corrosion (i.e. "real world" conditions.

10. Experimental studies of radiation damage processes to test fundamental mechanisms.

11. Large scale seismic facilities.

12. Understanding material processing history \& load history on SCC \& high temperature performance.

13. Local crystal plasticity effects on performance.

14. Remote NDE.

15. Effects of helium on materials properties.
Interested in Collaboration

Simon Pimblott Steve Donnelly Randy Nanstad Ian Hutchinson Ram Devanathan David Smith Tunc Aldemir John Bouchard

Bill Martin Alex Navrotsky Andrew Sherry Audeen Fentiman Brian Wirth George Smith Sudarshan Loyalka John Ireland Robin Grimes Martin Newby Chris Grovenor Paul Turinsky Jack Brenizer Todd Allen Roger Smith Peter Storey Ian Farnan 


\section{UK/US NUCLEAR ENGINEERING WORKSHOP \\ American Geophysical Union (AGU) \\ Washington DC \\ April 20- 21, 2009}

\subsection{Reactor Design, Operations, \& Monitoring}

\begin{tabular}{|c|c|c|}
\hline US Gaps/ Needs & UK Gaps/Needs & $\begin{array}{l}\text { Interested in } \\
\text { Collaboration }\end{array}$ \\
\hline 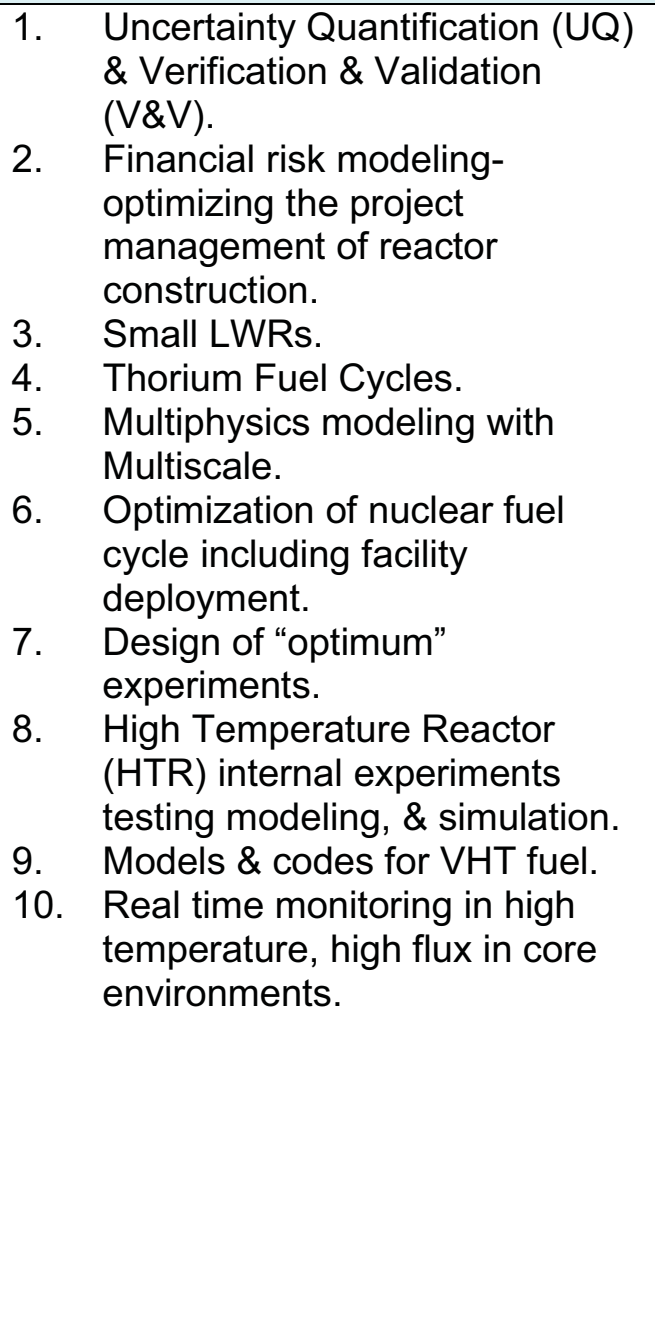 & 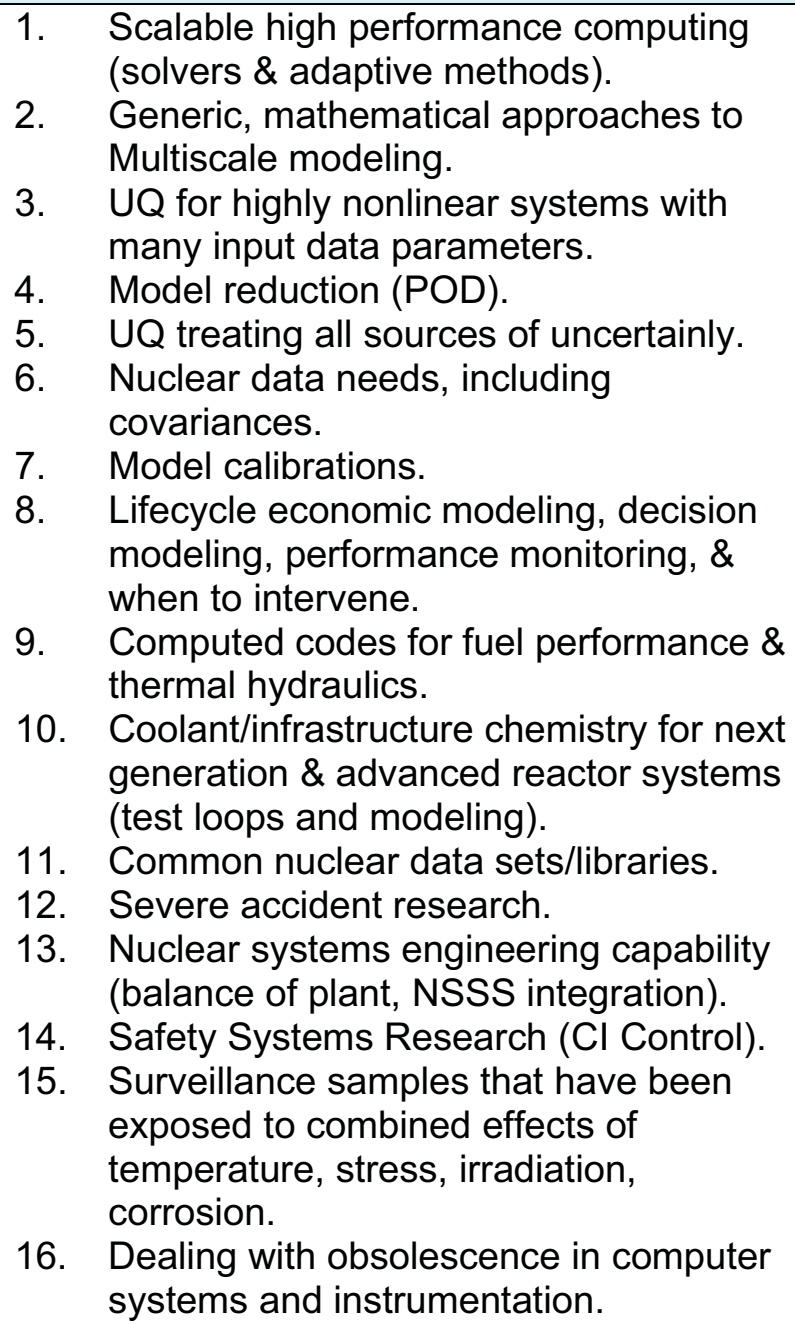 & $\begin{array}{l}\text { lan Farnan (for } \\
\text { Geoff Parks, Bill } \\
\text { Nutall) } \\
\text { Jack Brenizer } \\
\text { Tunc Aldemir } \\
\text { Martin Newby } \\
\text { lan Hutchinson } \\
\text { David Smith } \\
\text { Matt Eaton } \\
\text { Bill Martin } \\
\text { Sherrell Greene } \\
\text { Andrew Sherry } \\
\text { Audeen Fentiman } \\
\text { Andy Klein } \\
\text { Paul Turinsky (for } \\
\text { Dimetry Anistraton) } \\
\text { Jason Hayward } \\
\text { Simon Pimblott } \\
\text { John Ireland } \\
\text { Sudarshan Loyalka } \\
\text { George Smith } \\
\text { Igor Bodnar } \\
\text { Totju Totev } \\
\text { John Bouchard }\end{array}$ \\
\hline
\end{tabular}




\section{UK/US NUCLEAR ENGINEERING WORKSHOP \\ American Geophysical Union (AGU) \\ Washington DC \\ April 20- 21, 2009}

\subsection{SNF \& Waste Management}

\begin{tabular}{|ll|}
\hline \multicolumn{1}{|c|}{ US Gaps/ Needs } \\
\hline 1. & $\begin{array}{l}\text { Facilities for hot fuels experts plus } \\
\text { synchrotron light technologies. }\end{array}$ \\
2. & 'Nuclearised' analytical facilities. \\
3. & $\begin{array}{l}\text { Numerical modeling of multiphased transport } \\
\text { at multiple scales in highly heterogeneous } \\
\text { porous materials (including reactive transport } \\
\text { issues). }\end{array}$
\end{tabular}

4. Standard dataset and simulation approach to advanced fuel cycle analysis.

5. Fast ion implementation to stimulate radiation damage and coupled ion probe analysis techniques.

6. Hot cell capability to characterize microstructure of spent fuel cladding and test facility for corrosion studies in panel vector.

7. Well characterized/documented "Harvested" materials to help us understand long-term materials behavior/degradation.

8. In-situ examination of irradiated materials.

9. Interfacial processes (radiation-induced) of oxides / polymers, etc., at temperature and pressure.

10. Improved performance envelope for hydrometallurgical separation (e.g. third phase).

11. Long term strategic approach.

12. Chemistry of new/novel reprocessing systems.

13. Research on defined boundary conditions and endpoint, acceptance criteria between waste packages and repository.

14. Pilot scale/demonstration technology facilities, e.g. cold crucible melters.

15. Cements and grouts.

16. Public understanding of decision making using risk-based science.

17. Encapsulates for reactive metals.

18. Experimental verification of fission product partition in phases-ceramic waste forms.

19. Inert atmosphere capability to mimic repository conditions.

20. Non-aqueous processing technology.

21. Uncertainty elicitation - performance

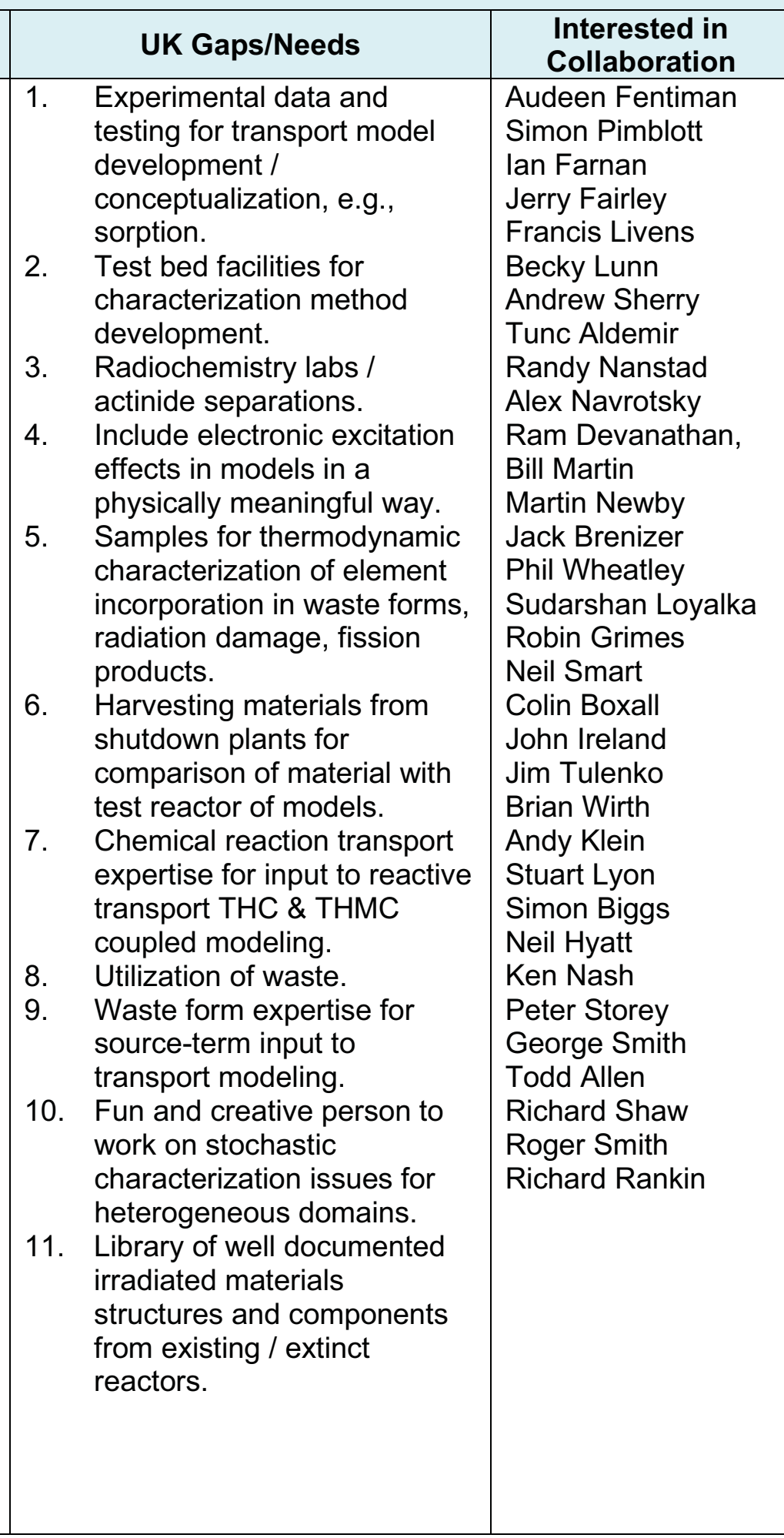




\section{UK/US NUCLEAR ENGINEERING WORKSHOP \\ American Geophysical Union (AGU) \\ Washington DC \\ April 20- 21, 2009}

\subsection{SNF \& Waste Management}

\begin{tabular}{|c|c|c|}
\hline US Gaps/ Needs & UK Gaps/Needs & $\begin{array}{l}\text { Interested in } \\
\text { Collaboration }\end{array}$ \\
\hline $\begin{array}{l}\text { monitoring - inspection - intervention. } \\
\text { 22. Propagation of risk in safety cases for } \\
\text { disposal / long-term storage. } \\
\text { 23. Hazard index for spent fuel inventory to } \\
\text { establish priorities for disposition. } \\
\text { 24. Behavior SF-storage/disposal. } \\
\text { 25. Waste product long-term performance. } \\
\text { 26. Pu disposition. } \\
\text { 27. U Storage and disposal } \\
\text { 28. Disposal site characterization. } \\
\text { 29. Experimental verification of fission product } \\
\text { behavior in vitreous waste forms. } \\
\text { 30. Are effects of beta, alpha, gamma, and } \\
\text { neutron radiations additive? }\end{array}$ & & \\
\hline
\end{tabular}

\subsection{Decommissioning and Decontamination}

\begin{tabular}{|c|c|c|}
\hline US Gaps/ Needs & UK Gaps/Needs & $\begin{array}{l}\text { Interested in } \\
\text { Collaboration }\end{array}$ \\
\hline 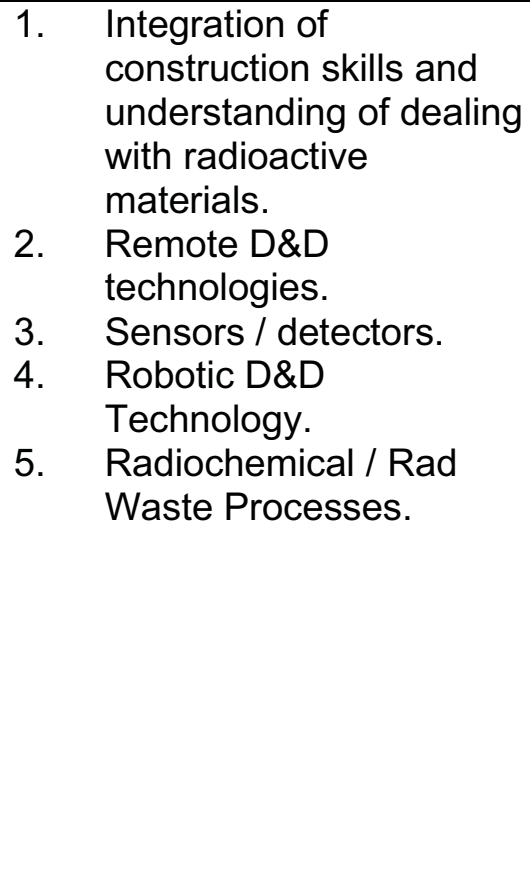 & $\begin{array}{l}\text { 1. Access to hot labs. } \\
\text { 2. } \begin{array}{l}\text { Engagement of appropriate academic skills base } \\
\text { (e.g., civil engineering). }\end{array} \\
\text { 3. Multi-criteria approach / life assessment to } \\
\text { decommission options. } \\
\text { 4. } \begin{array}{l}\text { Radiometric and non-radiometric characterization / } \\
\text { NDA techniques for determining radiation levels, hot } \\
\text { spots, etc., in facilities. }\end{array} \\
\text { 5. Scabbing concrete surfaces (clever concrete } \\
\text { decontamination). } \\
\text { 6. Low level analyses to support reclassification / free } \\
\text { 7. } \begin{array}{l}\text { Colease. } \\
\text { 8. Defined waste package boundary conditions and }\end{array} \\
\text { 9. } \begin{array}{l}\text { Refined site end point. } \\
\text { 10. Access to test materials / locations. }\end{array} \\
\text { 11. Have remote D\&D robotics. } \\
\text { 12. Public understanding of risk-based science and }\end{array}$ & $\begin{array}{l}\text { Audeen Fentiman } \\
\text { Neil Smart-NDA } \\
\text { Simon Biggs } \\
\text { Richard Shaw } \\
\text { Francis Livens (for } \\
\text { Bernard Kelly) } \\
\text { Richard Rankin } \\
\text { Colin Boxall } \\
\text { Phil Wheatley } \\
\text { Sherrell Greene } \\
\text { Andy Boston } \\
\text { Becky Lunn } \\
\text { Nick Stevens } \\
\text { Graham Fairhall } \\
\text { James Taylor } \\
\text { Colin Boxall }\end{array}$ \\
\hline
\end{tabular}




\section{UK/US NUCLEAR ENGINEERING WORKSHOP \\ American Geophysical Union (AGU) \\ Washington DC \\ April 20- 21, 2009}

\subsection{Safety, Security, Safeguards}

\begin{tabular}{|ll}
\hline \multicolumn{1}{|c}{ US Gaps/ Needs } \\
\hline 1. & $\begin{array}{l}\text { Identify high priority needs for } \\
\text { nuclear data relevant to nuclear } \\
\text { security and safeguards. }\end{array}$ \\
2. & $\begin{array}{l}\text { Low volume; high effective } \\
\text { detectors with detection and } \\
\text { identification capabilities. }\end{array}$
\end{tabular}

3. Data fusion to combine multiple sensors.

4. Wireless test.

5. Cyber security.

6. Proliferation Metrics.

7. Clear data sets.

8. International, common framework for risk informed decision making.

9. Security probabilistic risk assessment (PRA) technology.

10. Human Risk Analysis.

11. Probabilistic methods of safety Margins.

12. Licensing Confirmatory Analysis Capability for Advanced Reactors. (SFR, GCR, MSR)

13. Licensing Framework for Reprocessing Plants.

14. Monitoring and sensor development for high temperature/corrosive environments (and expertise in same for technology that exists.

15. Public understanding and regulation using risk-based science.

16. Larger volume, room temperature semiconductor sensors.

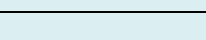




\section{UK/US NUCLEAR ENGINEERING WORKSHOP \\ American Geophysical Union (AGU) \\ Washington DC \\ April 20- 21, 2009}

\section{Team Development of Collaboration Opportunities Between the UK and the US}

\section{Group 1: Fuel Technology}

\begin{tabular}{|c|c|c|}
\hline Category: & \multicolumn{2}{|l|}{ 1. Fuel Technology } \\
\hline Title: & \multicolumn{2}{|c|}{ Modeling and Simulation of Fuel Fabrication } \\
\hline Opportunity: & \multicolumn{2}{|c|}{$\begin{array}{l}\text { Use modeling to explore well defined } n \text {-dimensional variable space to find } \\
\text { critical combinations of variables. } \\
\text { Multiscale, multiphysics addressing or predicting: } \\
\text { - Particle morphologies }-\mathrm{f}^{\mathrm{n}} \text { of process variables } \\
\text { - Flow and packing mixing characteristics (green body) } \\
\text { - Sintering models }\end{array}$} \\
\hline $\begin{array}{l}\text { Needs or Next } \\
\text { Steps: }\end{array}$ & \multicolumn{2}{|c|}{$\begin{array}{l}\text { Identify the funding stream. } \\
\text { Need non-ceramic fuel development. } \\
\text { Need help with sintering models. }\end{array}$} \\
\hline Country: & United Kingdom & United States \\
\hline Expertise: & $\begin{array}{l}\text { - Leeds - U-particle } \\
\text { - Imperial - morphology } \\
\text { - } \text { NNL - chemical engineering, } \\
\text { - Sheffield - ceramic processing, } \\
\text { microstructure }\end{array}$ & $\begin{array}{ll}\text { - } & \text { LANL - oxides/nitrides } \\
\text { - } & \text { ORNL - TRISO, actinide targets, } \\
\text { ceramics } \\
\text { - } \quad \text { INL - Dispersion, metal fuel (Rory } \\
\text { Kennedy) } \\
\text { - } \quad \text { Texas A\&M } \\
\text { - NC State University (Jacob Eapen) } \\
\text { - modeling \& simulation during } \\
\text { irradiation. }\end{array}$ \\
\hline Coordinators: & Scott Owens - NNL & Sherrell Green - ORNL \\
\hline
\end{tabular}

\begin{tabular}{|l|l|l|}
\hline Category: & 1. Fuel Technology \\
\hline Title: & Fuel Performance - Experimental \\
\hline Opportunity: & ATR \\
\hline $\begin{array}{l}\text { Needs or Next } \\
\text { Steps: }\end{array}$ & $\begin{array}{l}\text { New experiments and database of previous experimental results (Fuel } \\
\text { Performance Codes) }\end{array}$ \\
\hline Country: & \multicolumn{1}{|c|}{ United Kingdom } & \multicolumn{1}{|c|}{ United States } \\
\hline Expertise: & \multicolumn{1}{|c|}{} \\
\hline Coordinators: & lan Farnam & Todd Allen \\
\hline
\end{tabular}




\section{UK/US NUCLEAR ENGINEERING WORKSHOP \\ American Geophysical Union (AGU) \\ Washington DC \\ April 20- 21, 2009}

\begin{tabular}{|c|c|c|}
\hline Category: & \multicolumn{2}{|l|}{ 1. Fuel Technology } \\
\hline Title: & \multicolumn{2}{|c|}{ Experimental Verification of Fuel Fabrication Steps } \\
\hline Opportunity: & \multicolumn{2}{|c|}{$\begin{array}{l}\text { Crossover to many modeling steps. } \\
\text { Test - morphology changes at confluence of variables that exhibit critical } \\
\text { change: validate. } \\
\text { Test - the models - develop process models. } \\
\text { Test - predictions of porosity, mechanical properties, etc. }\end{array}$} \\
\hline \multicolumn{3}{|l|}{$\begin{array}{l}\text { Needs or Next } \\
\text { Steps: }\end{array}$} \\
\hline Country: & United Kingdom & United States \\
\hline Expertise: & $\begin{array}{ll}\text { - } & \text { NNL } \\
\text { - } & \text { LEEDS - Biggs } \\
\text { - } & \text { Manchester } \\
\text { - } & \text { Imperial - Lee } \\
\text { - } & \text { Sheffield }\end{array}$ & $\begin{array}{l}\text { - INL-Rory Kennedy \& Steve Hayes } \\
\text { - Illinois } \\
\text { ORNL - Sol Gel, sphere pack, } \\
\text { TRISO (LEU, Actinide), Ceramic } \\
\text { (actinide targets, U-Pu-NE), VAL } \\
\text { dispersion/CERMET } \\
\text { - } \text { LANL - UO2, MOX }\end{array}$ \\
\hline Coordinators: & Tim Abram & Brent Heuser \\
\hline
\end{tabular}

\begin{tabular}{|c|c|c|}
\hline Category: & \multicolumn{2}{|l|}{ 1. Fuel Technology } \\
\hline Title: & \multicolumn{2}{|c|}{ Fuel Performance - Modeling \& Simulation } \\
\hline Opportunity: & \multicolumn{2}{|c|}{$\begin{array}{l}\text { Extensive programs underway in Ezah Country. Seeking to enhance } \\
\text { contributions by coordination and making people aware of other programs. }\end{array}$} \\
\hline $\begin{array}{l}\text { Needs or Next } \\
\text { Steps: }\end{array}$ & \multicolumn{2}{|c|}{$\begin{array}{l}\text { Mechanisms to coordinate } \\
\text { 1. Develop e-mail list (keep open for new additions) } \\
\text { 2. RG/JT to meet on May } 20 \text { to continue formation } \\
\text { 3. Utilize CMSN/NEA to coordinate with other entities. }\end{array}$} \\
\hline Country: & United Kingdom & United States \\
\hline Expertise: & $\begin{array}{l}\text { - I.C. - Cambridge } \\
\text { - M.C.-Oxford }\end{array}$ & $\begin{array}{ll}- & \text { INL-LANL-UF } \\
- & \text { ELNL-PNNL-UCB } \\
- & \text { ANL-ORNL-MIT }\end{array}$ \\
\hline Coordinators: & Robin Grimes (RG) & Jim Tulenko (JT) \\
\hline
\end{tabular}




\section{UK/US NUCLEAR ENGINEERING WORKSHOP \\ American Geophysical Union (AGU) \\ Washington DC \\ April 20- 21, 2009}

\section{Group 2: Reactor Materials Performance}

\begin{tabular}{|c|c|c|}
\hline Category: & \multirow{2}{*}{\multicolumn{2}{|c|}{$\begin{array}{l}\text { 2. Reactor Materials Performance } \\
\text { Irradiation Embrittlement - Covers RPU and structural materials }\end{array}$}} \\
\hline Title: & & \\
\hline Opportunity: & \multicolumn{2}{|c|}{$\begin{array}{l}\text { - Life extension } \\
\text { - } \quad \text { Provision of US facilities } \\
\text { - Expansion of multiscale modeling basic physics }\end{array}$} \\
\hline $\begin{array}{l}\text { Needs or Next } \\
\text { Steps: }\end{array}$ & \multicolumn{2}{|c|}{$\begin{array}{l}\text { Define scope, needs, and money. } \\
\text { Coordinators will define working group. }\end{array}$} \\
\hline Country: & United Kingdom & United States \\
\hline Expertise: & $\begin{array}{l}\text { Liverpool, Oxford, Loughborough, } \\
\text { Imperial, Manchester, Salford. }\end{array}$ & $\begin{array}{l}\text { INL, ORNL, LANL (flux rate effects) } \\
\text { Michigan, Berkeley, Davis, LANL, } \\
\text { Wisconsin, Santa Barbara. U of } \\
\text { Michigan (Gary Was) } \\
\text { Fei Gao (PNNL) is working on } \\
\text { simulations relevant to lifetime } \\
\text { extension (fei.gao@ @pnl.gov). } \\
\text { NC State University - experimental } \\
\text { work on Zirconium based fuel cladding } \\
\text { and carbon steel reactor pressure } \\
\text { vessels (K.L. Murty). } \\
\text { Penn State University - Arthur Motta - } \\
\text { irradiation damage. }\end{array}$ \\
\hline Coordinators: & Steve Donnelly / Randy Grimes & Brian Wirth / Todd Allen \\
\hline
\end{tabular}

\begin{tabular}{|c|c|c|}
\hline Category: & \multicolumn{2}{|c|}{ 2. Reactor Materials Performance } \\
\hline Title: & \multicolumn{2}{|c|}{ Library - Material Harvesting (refer to group 4) } \\
\hline Opportunity: & \multicolumn{2}{|c|}{ Database/ characterization of materials. } \\
\hline $\begin{array}{l}\text { Needs or Next } \\
\text { Steps: }\end{array}$ & \multicolumn{2}{|c|}{ Group 4 (waste) and Group 1 ( Fuels) } \\
\hline Country: & United Kingdom & United States \\
\hline \multicolumn{3}{|l|}{ Expertise: } \\
\hline Coordinators: & Graham Fairhall - NNL & $\begin{array}{l}\text { Allen, Nanstad, Maloy (ANL, ORNL, } \\
\text { LANL) }\end{array}$ \\
\hline
\end{tabular}




\section{UK/US NUCLEAR ENGINEERING WORKSHOP \\ American Geophysical Union (AGU) \\ Washington DC \\ April 20- 21, 2009}

\begin{tabular}{|c|c|c|}
\hline Category: & \multicolumn{2}{|l|}{ 2. Reactor Materials Performance } \\
\hline Title: & \multicolumn{2}{|c|}{ High Temperature Structural Materials (Metals and Non-Metals) } \\
\hline Opportunity: & \multicolumn{2}{|c|}{$\begin{array}{l}\text { Overlaps with fusion. } \\
\text { Access to facilities, including irradiation } \\
\text { Fabrication processes. } \\
\text { Life extension. } \\
\text { Potential for links with conventional power industry's interest in high } \\
\text { temperature materials (David Smith - Bristol). }\end{array}$} \\
\hline $\begin{array}{l}\text { Needs or Next } \\
\text { Steps: }\end{array}$ & \multicolumn{2}{|c|}{$\begin{array}{l}\text { Define scope, needs, dosh-funding. } \\
\text { Coordinators will define the working groups. } \\
\text { Scope: need to understand combined effects of stress, temperature, and } \\
\text { radiation on structural materials. }\end{array}$} \\
\hline Country: & United Kingdom & United States \\
\hline Expertise: & Bristol, Oxford, Open, Imperial, & $\begin{array}{l}\text { ORNL, INL, LANL, Boise State, Wis, } \\
\text { MIT (fusion), Michigan, Davis, Bartel, } \\
\text { Gary Was (U of Michigan). } \\
\text { High temperature structural materials } \\
\text { (non-metal) e.g. SiC, carbides - Ram } \\
\text { Devanathan (PNNL) } \\
\text { Ram.devanathan@pnl.gov }\end{array}$ \\
\hline Coordinators: & $\begin{array}{l}\text { D. Smith (metals), Marsden (non- } \\
\text { metals) }\end{array}$ & $\begin{array}{l}\text { Nanstad (metals), Lance Snead (non- } \\
\text { metals) (ORNL) }\end{array}$ \\
\hline
\end{tabular}

\begin{tabular}{|c|c|c|}
\hline Category: & \multicolumn{2}{|l|}{ 2. Reactor Materials Performance } \\
\hline Title: & \multicolumn{2}{|c|}{ PWSCC and Irradiation (EAC) - Covers steels, cladding, Zirconium } \\
\hline Opportunity: & \multicolumn{2}{|c|}{$\begin{array}{l}\text { US facilities for irradiation and post testing analysis impact of cold work, } \\
\text { mechanistic understanding, etc. }\end{array}$} \\
\hline $\begin{array}{l}\text { Needs or Next } \\
\text { Steps: }\end{array}$ & \multicolumn{2}{|c|}{$\begin{array}{l}\text { Define scope, needs, and funding. } \\
\text { Coordinators will define the working group. } \\
\text { Links - Group } 4 \text { waste containment. }\end{array}$} \\
\hline Country: & United Kingdom & United States \\
\hline Expertise: & Manchester, Oxford, Open, Bristol & $\begin{array}{l}\text { INL, ORNL, PNNL, ANL, LANL, } \\
\text { Michigan, Wisconsin, MIT } \\
\text { Penn State University - Arthur Motta - } \\
\text { stress crack corrosion }\end{array}$ \\
\hline Coordinators: & $\begin{array}{l}\text { James Marrow (Mandi), Simon } \\
\text { Pimblott }\end{array}$ & Jeremy Busby - ORNL \\
\hline
\end{tabular}




\section{UK/US NUCLEAR ENGINEERING WORKSHOP \\ American Geophysical Union (AGU) \\ Washington DC \\ April 20- 21, 2009}

\section{Group 3. Reactor Design, Operations, Monitoring, and Safety}

\begin{tabular}{|l|l|l|}
\hline Category: & 3. Reactor Design, Operations, Monitoring, and Safety \\
\hline Title: & $\begin{array}{l}\text { Uncertainty quantification for highly non-linear systems, including data } \\
\text { assimilation and design of experiments. (includes aging phenomena) }\end{array}$ \\
\hline Opportunity: & \multicolumn{1}{|c|}{ United Kingdom } & \multicolumn{1}{|c|}{ United States } \\
\hline $\begin{array}{l}\text { Needs or Next } \\
\text { Steps: }\end{array}$ & $\begin{array}{l}\text { Need to understand impact of uncertainty on lifetime prediction (David Smith } \\
\text { Link to Group 2. }\end{array}$ \\
\hline Country: & \multicolumn{1}{|c|}{ Unitol } \\
\hline Expertise: & $\begin{array}{l}\text { Geoff Parks (Cambridge) } \\
\text { Coordinators: }\end{array}$ Martin Newby & Paul Turinsky \\
\hline
\end{tabular}

\begin{tabular}{|l|l|l|}
\hline Category: & 3. Reactor Design, Operations, Monitoring, and Safety \\
\hline Title: & Generation of Nuclear Data \\
\hline Opportunity: & $\begin{array}{l}\text { Generation of nuclear data via differential and integral experiments, and } \\
\text { utilization in nuclear models. }\end{array}$ \\
\hline $\begin{array}{l}\text { Needs or Next } \\
\text { Steps: }\end{array}$ & \multicolumn{1}{|c|}{ United Kingdom } & \\
\hline Country: & $\begin{array}{l}\text { Manchester nuclear physics - Jon } \\
\text { Billowes } \\
\text { EK-NNL - looking at national } \\
\text { needs for nuclear data in UK - } \\
\text { Colin Zimmerman } \\
\text { Interested in thorium and } \\
\text { accelerator driven systems - Bill } \\
\text { Nuttall - Cambridge }\end{array}$ & \\
\hline Coordinators: & Matthew Eaton & Paul Turinsky \\
\hline
\end{tabular}




\section{UK/US NUCLEAR ENGINEERING WORKSHOP \\ American Geophysical Union (AGU) \\ Washington DC \\ April 20- 21, 2009}

\begin{tabular}{|c|c|c|}
\hline Category: & \multicolumn{2}{|c|}{ 3. Reactor Design, Operations, Monitoring, and Safety } \\
\hline Title: & \multicolumn{2}{|l|}{ I\&C } \\
\hline Opportunity: & \multicolumn{2}{|c|}{$\begin{array}{l}\text { Issues associated with instrumentation and control for operating reactors and } \\
\text { Gen IV. }\end{array}$} \\
\hline \multicolumn{3}{|l|}{$\begin{array}{l}\text { Needs or Next } \\
\text { Steps: }\end{array}$} \\
\hline Country: & United Kingdom & United States \\
\hline Expertise: & $\begin{array}{l}\text { Requirement for statistical } \\
\text { testing of software - John Ray. } \\
\text { - Statistical Software Analysis - } \\
\text { Pete Sorop (?), Malcohm Lople } \\
\text { (Lancaster) }\end{array}$ & \\
\hline Coordinators: & John May (Bristol) & Tunc Aldemir \\
\hline
\end{tabular}

\begin{tabular}{|l|l|l|}
\hline Category: & 3. Reactor Design, Operations, Monitoring, and Safety \\
\hline Title: & Fundamentally based modeling for severe accidents. \\
\hline Opportunity: & \multicolumn{2}{|c|}{} \\
\hline $\begin{array}{l}\text { Needs or Next } \\
\text { Steps: }\end{array}$ & $\begin{array}{l}\text { Link this to education and training using advanced reactor simulators. Can } \\
\text { these be made available for student education and training? }\end{array}$ \\
\hline Country: & \multicolumn{2}{|c|}{ United Kingdom } \\
\hline Expertise: & $\begin{array}{l}\text { Newcastle, UNI, UK - Involved in } \\
\text { SARNET \& interested in this area. }\end{array}$ & \\
\hline Coordinators: & Matthew Eaton & Andy Klein \\
\hline
\end{tabular}

\begin{tabular}{|l|l|l|}
\hline Category: & $\begin{array}{l}\text { 3. Reactor Design, Operations, Monitoring, and Safety } \\
\text { Self-adaptive, goal-oriented (e.g., risk or funding) modeling approaches } \\
\text { using algorous multi-scale closure relations. }\end{array}$ \\
\hline Title: & \multicolumn{2}{|l|}{} \\
\hline Opportunity: & \multicolumn{2}{|l|}{ United States } \\
\hline $\begin{array}{l}\text { Needs or Next } \\
\text { Steps: }\end{array}$ & \multicolumn{1}{|l|}{ United Kingdom } & \multicolumn{1}{|l}{} \\
\hline Country: & \multicolumn{2}{|l|}{ Bill Martin } \\
\hline Expertise: & Matthew Eaton &
\end{tabular}




\section{UK/US NUCLEAR ENGINEERING WORKSHOP \\ American Geophysical Union (AGU) \\ Washington DC \\ April 20- 21, 2009}

\section{Group 4: SNF \& Waste Management}

\begin{tabular}{|c|c|c|}
\hline Category: & \multicolumn{2}{|l|}{ 4. SNF \& Waste Management } \\
\hline Title: & \multicolumn{2}{|c|}{ Waste system performance assessment modeling } \\
\hline Opportunity: & \multicolumn{2}{|c|}{$\begin{array}{l}\text { Integrating coupled processes that exhibit complex behavior at a wide range } \\
\text { of scales. } \\
\text { - How can Multiscale models be applied to engineered and natural barrier } \\
\text { systems? } \\
\text { - How do we model the interactions between radionuclides, mineralogy and } \\
\text { microbial populations in a thermal-hydro-mechanical framework? } \\
\text { - How can we bound and reduce critical sources of uncertainty in prediction } \\
\text { of waste system performance? }\end{array}$} \\
\hline $\begin{array}{l}\text { Needs or Next } \\
\text { Steps: }\end{array}$ & \multicolumn{2}{|c|}{$\begin{array}{l}\text { - Contact key individuals that can bring expertise from other fields } \\
\text { (hydrocarbon, CO2 sequestration geo-microbiology, rock structure, } \\
\text { chemistry, etc.) } \\
\text { - Hold a 1-2 day workshop to build collaborative US/UK research teams to } \\
\text { address proposed research questions. }\end{array}$} \\
\hline Country: & United Kingdom & United States \\
\hline Expertise: & $\begin{array}{l}\text { Don't forget that BGS has work } \\
\text { programming in CCS, hydrocarbon, } \\
\text { and resources areas that are } \\
\text { relevant. RP Smith (?) } \\
\text { Kath Morris - Leeds } \\
\text { Richard Pattrick - Manchester } \\
\text { Nick Bryan, Jon Lloyd - } \\
\text { Manchester } \\
\text { Andy Binley - Ground water flow } \\
\text { around repository (Lancaster ) }\end{array}$ & $\begin{array}{l}\text { PNNL has expertise in CO2 } \\
\text { sequestration, geomicrobiology, and } \\
\text { reactive transport needed. - EMSL } \\
\text { facility at PNNL } \\
\text { Is there a need or potential for } \\
\text { understanding He structural integrity } \\
\text { and life of waste containment systems? } \\
\text { David Smith (Bristol) }\end{array}$ \\
\hline Coordinators: & Rebecca Lunn & Jerry Fairley \\
\hline
\end{tabular}

\begin{tabular}{|l|l|}
\hline Category: & 4. SNF \& Waste Management \\
\hline Title: & Understanding Processes in Waste Forms in Waste Forms - Group 4 \\
\hline Opportunity: & $\begin{array}{l}\text { Structural and thermodynamic characterization and kinetics. } \\
\text { Model relevant experimental conditions. } \\
\text { Bridging scales of model } \\
\text { Interfacial phenomena } \\
\text { Separating thermal and radiation effects } \\
\text { Identify specific waste forms. } \\
\text { Take advantage of advances in computing } \\
\text { Multiple characterization techniques. }\end{array}$ \\
\hline
\end{tabular}




\section{UK/US NUCLEAR ENGINEERING WORKSHOP \\ American Geophysical Union (AGU) \\ Washington DC \\ April 20- 21, 2009}

\begin{tabular}{|l|l|l|}
\hline $\begin{array}{l}\text { Needs or Next } \\
\text { Steps: }\end{array}$ & $\begin{array}{l}\text { Identify specific systems to study } \\
\text { Exchange samples and people } \\
\text { Special session at MRS 2010 spring } \\
\text { Logistics } \\
\text { Support for making, characterizing, sharing (shipping) samples. }\end{array}$ \\
\hline Country: & \multicolumn{1}{|c|}{ United Kingdom } & \multicolumn{1}{c|}{ United States } \\
\hline Expertise: & $\begin{array}{l}\text { Understanding processes in waste } \\
\text { forms interfacial phenomena. } \\
\text { Water uptake on metal oxide } \\
\text { surfaces - Colin Boxall - Lancaster } \\
\text { Nick Bryan, Manchester, Francis } \\
\text { Livens, Simon Pimblott, }\end{array}$ & Lumin Wang, Univ of Michigan \\
\hline Coordinators: & \begin{tabular}{l} 
lan Farnan (Cambridge) \\
\hline
\end{tabular} & Ram Devanathan (PNNL) \\
\hline
\end{tabular}

\begin{tabular}{|c|c|c|}
\hline Category: & \multicolumn{2}{|l|}{ 4. SNF \& Waste Management } \\
\hline Title: & \multicolumn{2}{|l|}{ Performance of Irradiated materials } \\
\hline Opportunity: & \multicolumn{2}{|c|}{$\begin{array}{l}\text { Complimentary expertise at UofM/INL } \\
\text { - Coordination of future programs } \\
\text { - Comparisons of lessons learned } \\
\text { Fuels, oxides, metals, nitrides, oxides, structural materials, metals, graphite }\end{array}$} \\
\hline $\begin{array}{l}\text { Needs or Next } \\
\text { Steps: }\end{array}$ & \multicolumn{2}{|c|}{$\begin{array}{l}\text { Define matrix of expertise } \\
\text { Define mechanism for exchange of personnel } \\
\text { Build linked research programs. } \\
\text { Note: Cross over with waste forms for irradiation studies }\end{array}$} \\
\hline Country: & United Kingdom & United States \\
\hline Expertise: & $\begin{array}{l}\text { Qualification of radiation damage; } \\
\text { number of atoms displaced - lan } \\
\text { Farnan - Cambridge } \\
\text { Performance of irradiated } \\
\text { materials; water uptake on plukowii } \\
\text { (?) surfaces - Colin Boxall } \\
\text { Radiation effects in carbides; } \\
\text { oxides - Ram Devanathan (PNNL) } \\
\text { Interested in modeling atomic level } \\
\text { radiation effects and defect } \\
\text { evolution. }\end{array}$ & Lumin Wang $-U$ of Michigan \\
\hline Coordinators: & Simon Pimblott (+MPC) & Philip Wheatley \\
\hline
\end{tabular}




\section{UK/US NUCLEAR ENGINEERING WORKSHOP \\ American Geophysical Union (AGU) \\ Washington DC \\ April 20- 21, 2009}

\begin{tabular}{|c|c|c|}
\hline Category: & \multicolumn{2}{|c|}{ 4. SNF \& Waste Management } \\
\hline Title: & \multicolumn{2}{|c|}{$\begin{array}{l}\text { Sample harvesting for lifetime extension and performance of materials } \\
\text { in storage }\end{array}$} \\
\hline Opportunity: & \multicolumn{2}{|c|}{$\begin{array}{l}\text { Reciprocal access to inventions of well characterized samples. } \\
\text { Exploit pooled characterization tools. Generate new data to link to predictive } \\
\text { capabilities. }\end{array}$} \\
\hline $\begin{array}{l}\text { Needs or Next } \\
\text { Steps: }\end{array}$ & \multicolumn{2}{|c|}{$\begin{array}{l}\text { NNL/INL/ANL/ORNL to meet and link activities. } \\
\text { Develop plan to define the way forward. This needs buy in from national } \\
\text { regulators and industries. } \\
\text { Coordinate harvesting with group } 2 . \\
\text { Action Plan: } \\
\text { - Construct matrix of interests, participants, and links. } \\
\text { - Develop strategy and research priorities. } \\
\text { - Present coordinated objectives and routes to success. } \\
\text { - } \quad \text { Utilize synergies to optimize program. } \\
\text { - Seek seed funding for workshop and exchange visits } \\
\text { - } \quad \text { Coverage progress - feasibility. }\end{array}$} \\
\hline Country: & United Kingdom & United States \\
\hline \multicolumn{3}{|l|}{ Expertise: } \\
\hline Coordinators: & Graham Fairhall & Phil Wheatley \\
\hline
\end{tabular}

\begin{tabular}{|l|l|}
\hline Category: & 4. SNF \& Waste Management \\
\hline Title: & Advanced Partitioning and Separations \\
\hline Opportunity: & $\begin{array}{l}\text { Simplification of processing schemes } \\
\text { Improving Rad stability of materials } \\
\text { Gen IV support } \\
\text { Spent fuel/orphan waste disposition }\end{array}$ \\
\hline $\begin{array}{l}\text { Needs or Next } \\
\text { Steps: }\end{array}$ & $\begin{array}{l}\text { Understand underpinning - Chemistry of existing schemes } \\
\text { Development of new schemes (simplified) } \\
\text { Development of new schemes (improved Rad Stability) } \\
\text { Mechanics } \\
\text { Discussions between NL and academic partners in both US/UK } \\
\text { NL/NDA discussions in UK } \\
\text { Mechanism and Action Plan Detail: } \\
\text { - Richard Taylor, Colin Boxall to meet with Robin Taylor } \\
\text { - Richard Taylor, Colin Boxall to meet with Francis Livens and Simon } \\
\text { Pimblott. }\end{array}$ \\
\hline
\end{tabular}




\section{UK/US NUCLEAR ENGINEERING WORKSHOP \\ American Geophysical Union (AGU) \\ Washington DC \\ April 20- 21, 2009}

\begin{tabular}{|c|c|c|}
\hline & \multicolumn{2}{|c|}{$\begin{array}{l}\text { - Ken Nash to meet with Gregg Lumetta (PNNL) } \\
\text { - Ken Nash to meet with Scott Herbst (INL) } \\
\text { - Notes to be compared } \\
\text { - } \quad \text { Kiscussions with NDA as key NNL customer } \\
\text { Output Target: Outline research plan addressing all stakeholder needs. } \\
\text { Means: Exploitation of existing resolve to facilitate plan development } \\
\text { meeting. } \\
\text { Multiple established dialogs; informal collaborations between ORNL \& UK. } \\
\text { Should survey other labs as well. Sherrell Greene }\end{array}$} \\
\hline Country: & United Kingdom & United States \\
\hline Expertise: & $\begin{array}{l}\text { Physical-chemistry modeling, } \\
\text { Actinide chemistry, separation } \\
\text { science } \\
\text { Sven Schroeder (Manchester } \\
\text { Chem. Engineering). Contact via } \\
\text { F. Livens - Process Development }\end{array}$ & $\begin{array}{l}\text { Actinide chemistry; separations science } \\
\text { US Universities: UNLV, Oregon State }\end{array}$ \\
\hline Coordinators: & Colin Boxall - Lancaster & Ken Nash, WSU \\
\hline
\end{tabular}




\section{UK/US NUCLEAR ENGINEERING WORKSHOP \\ American Geophysical Union (AGU) \\ Washington DC \\ April 20- 21, 2009}

\section{Group 5: Decommissioning and Decontamination (D\&D)}

\begin{tabular}{|l|l|l|}
\hline Category: & 5. Decommissioning and Decontamination (D\&D) \\
\hline Title: & Measurement for D\&D \\
\hline Opportunity: & Better, faster, cheaper, safer, analysis, especially non-penetrating. \\
\hline $\begin{array}{l}\text { Needs or Next } \\
\text { Steps: }\end{array}$ & $\begin{array}{l}\text { Utilize measurement, signal processing, and deployment. } \\
\text { Further development of ideas. ASAP identify specific target application. }\end{array}$ \\
\hline Country: & \multicolumn{1}{|c|}{ United Kingdom } & \multicolumn{1}{|c|}{ United States } \\
\hline Expertise: & $\begin{array}{l}\text { Bristol - Potential research on civil } \\
\text { engineering deconstruction of } \\
\text { structures - David Smith } \\
\text { NNL - Sellafield Requirements. } \\
\text { Liverpool - Instruments - A. }\end{array}$ & Boston, P. Nolan \\
\hline Coordinators: & Malcolm Joyce & Richard Rankin \\
\hline
\end{tabular}

\begin{tabular}{|l|l|l|}
\hline Category: & 5. Decommissioning and Decontamination (D\&D) \\
\hline Title: & Knowledge and Technology Transfer \\
\hline Opportunity: & $\begin{array}{l}\text { Improvement in D\&D techniques, waste characterization, and volume } \\
\text { reduction. } \\
\text { Understanding of problems and fresh insight. }\end{array}$ \\
\hline $\begin{array}{l}\text { Needs or Next } \\
\text { Steps: }\end{array}$ & \begin{tabular}{l} 
Follow-on Workshop(s) \\
\hline Country:
\end{tabular} & \multicolumn{1}{|c|}{ United Kingdom } \\
\hline Expertise: & \multicolumn{1}{|c|}{ United States } \\
\hline Coordinators: & Bernard Kelly & Richard Rankin \\
\hline
\end{tabular}


옴

ํํำ

บ.

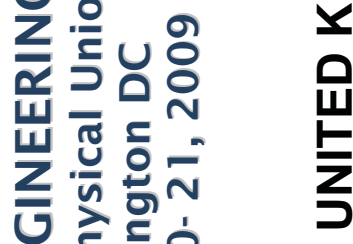

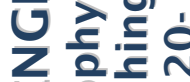

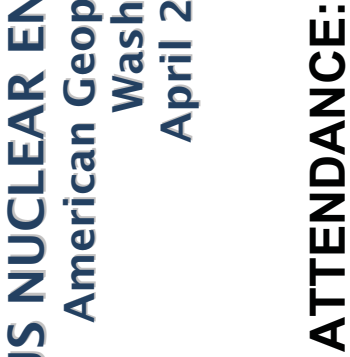

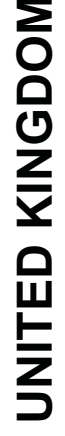

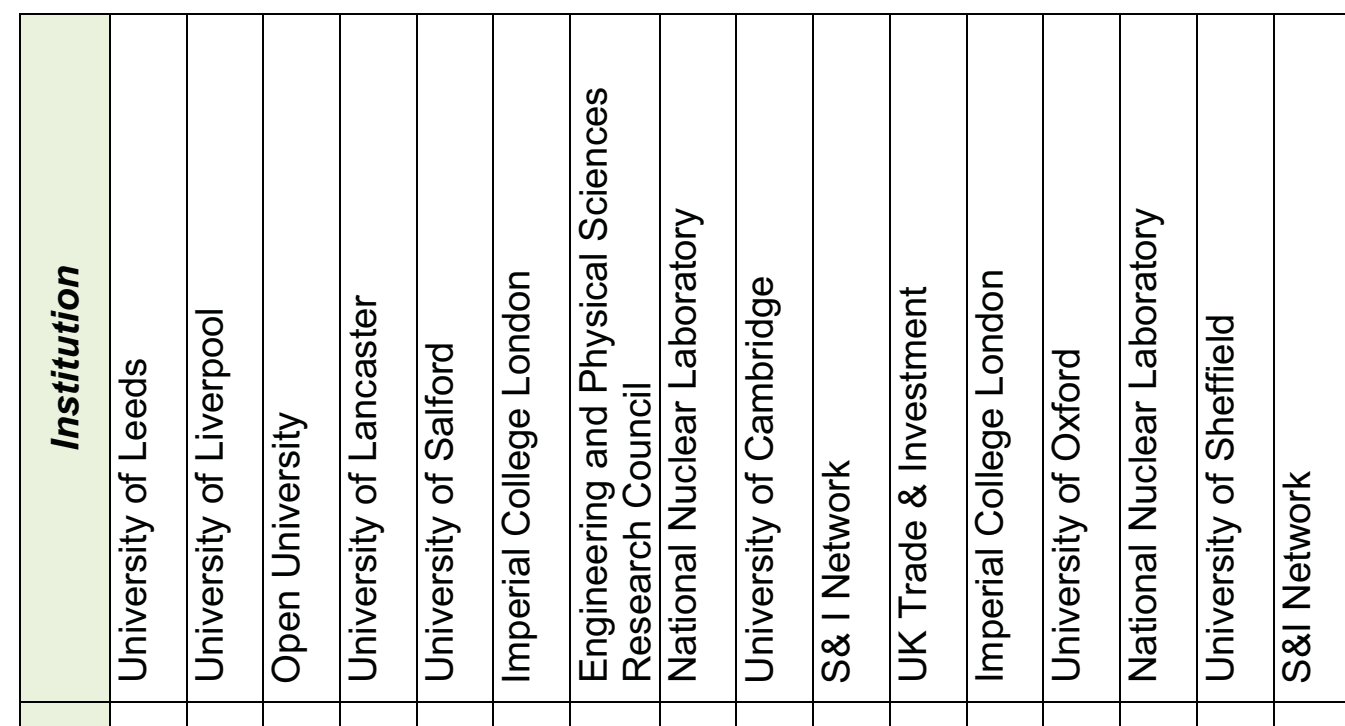

当

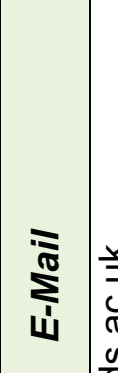

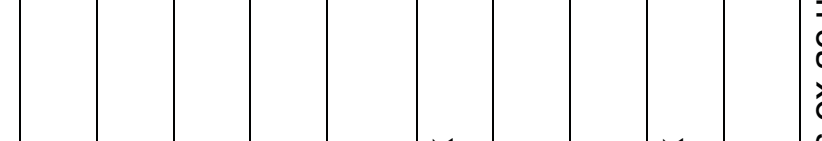

喜 兰

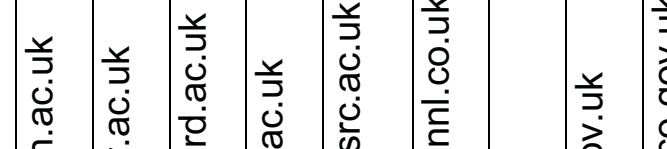

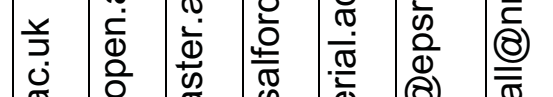

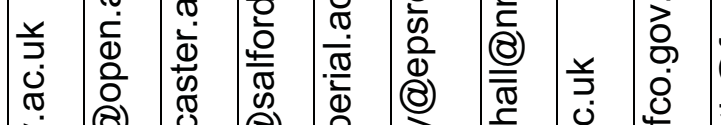

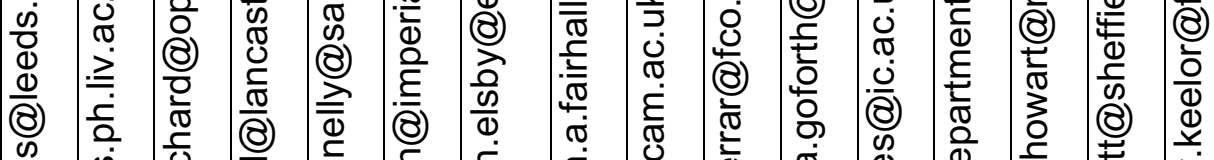

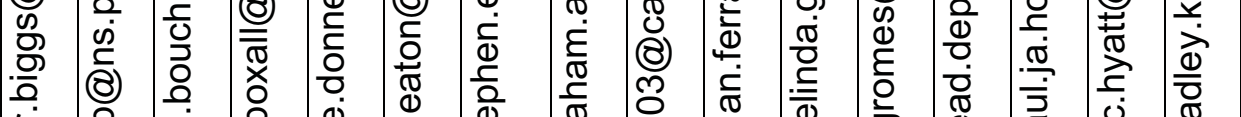

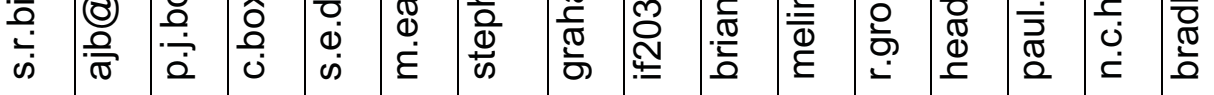

이

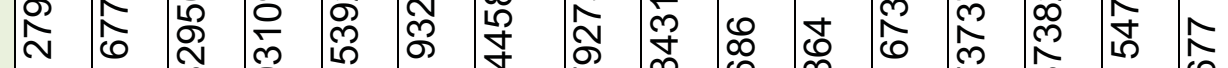

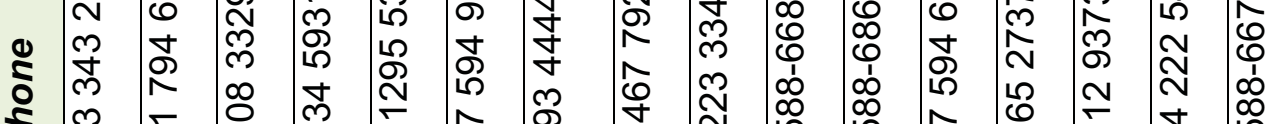
ฉ

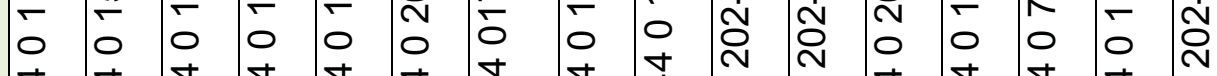

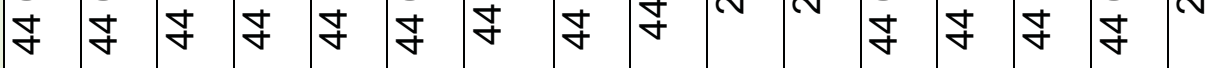

\begin{tabular}{|c|c|c|c|c|c|c|c|c|c|c|c|c|c|c|c|}
\hline 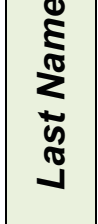 & $\frac{n}{g}$ & $\begin{array}{l}\text { D } \\
\text { क्. } \\
0 \\
0 \\
0\end{array}$ & 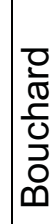 & $\begin{array}{l}\overline{\overline{\widetilde{\varpi}}} \\
\text { } \\
\varnothing \\
\emptyset\end{array}$ & 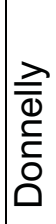 & 号 & $\frac{\widehat{\partial}}{\frac{0}{\omega}}$ & 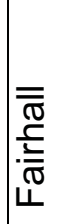 & 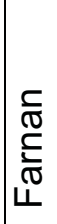 & 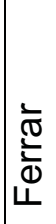 & 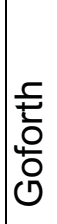 & 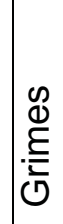 & $\begin{array}{l}\frac{0}{0} \\
\frac{0}{10} \\
\frac{0}{0}\end{array}$ & 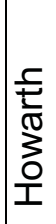 & 芜 \\
\hline 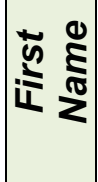 & $\frac{\check{c}}{\mathrm{O}}$ & $\begin{array}{l}\frac{3}{d} \\
\frac{1}{0} \\
\frac{1}{2} \\
\end{array}$ & 동 & ป气 & 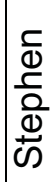 & 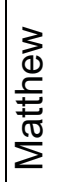 & $\begin{array}{l}\frac{c}{d} \\
\frac{c}{0} \\
\frac{0}{d} \\
\text { c) }\end{array}$ & $\begin{array}{l}\frac{E}{\widetilde{D}} \\
\frac{C}{\widetilde{N}} \\
\frac{\mathbb{U}}{\mathbb{1}}\end{array}$ & 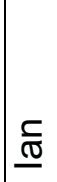 & 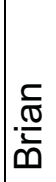 & 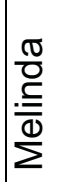 & \begin{tabular}{l}
$: \frac{}{0}$ \\
$\overline{0}$ \\
\hdashline
\end{tabular} & 先 & $\begin{array}{l}\overline{\widetilde{\sigma}} \\
\alpha\end{array}$ & $\overline{\bar{\theta}}$ \\
\hline
\end{tabular}



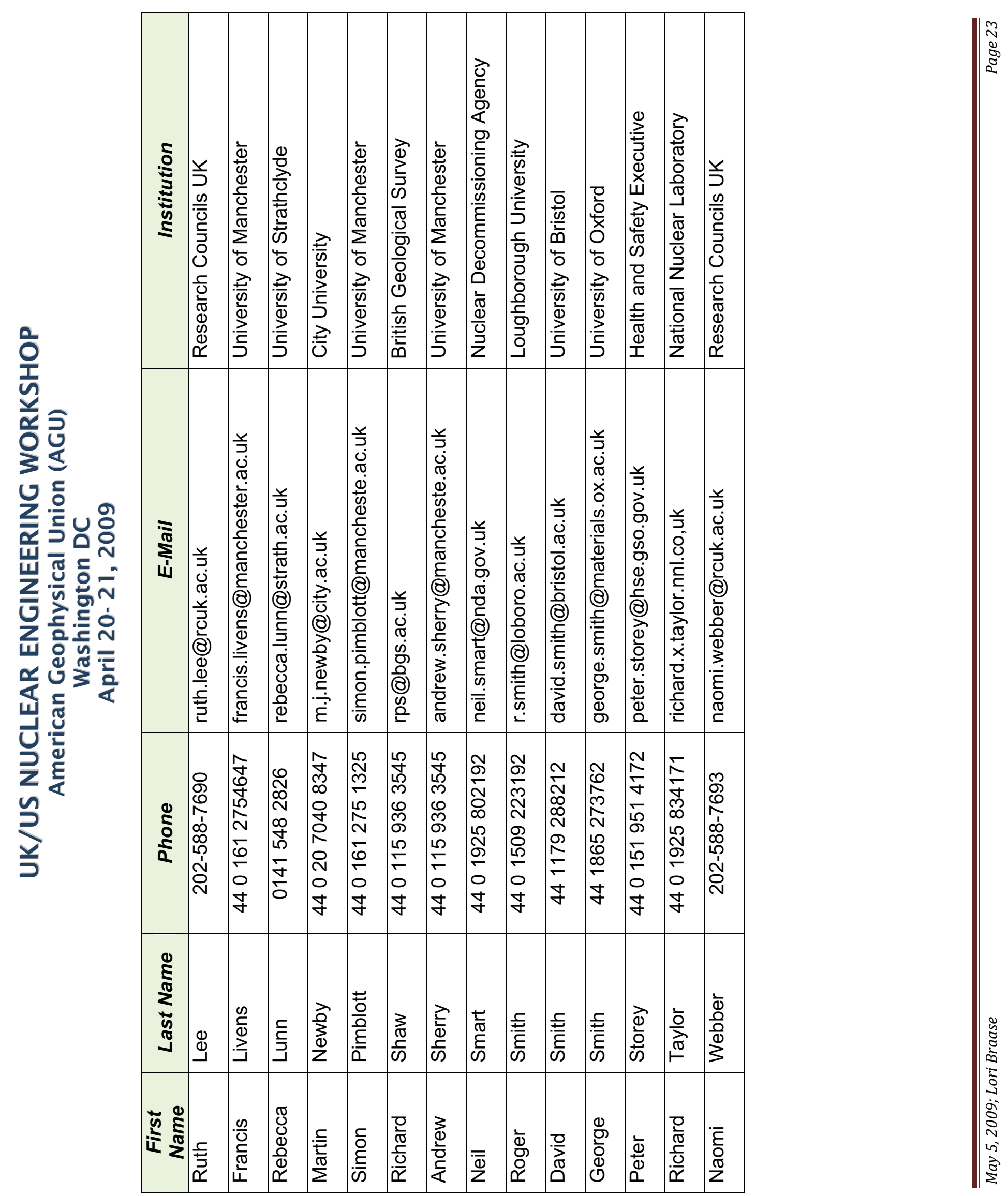
홈

วิํํำ

U.

르릉ㅇㅇ

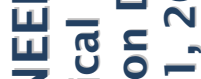

政

vis.

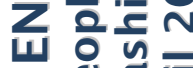

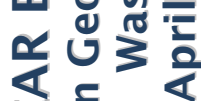

竞

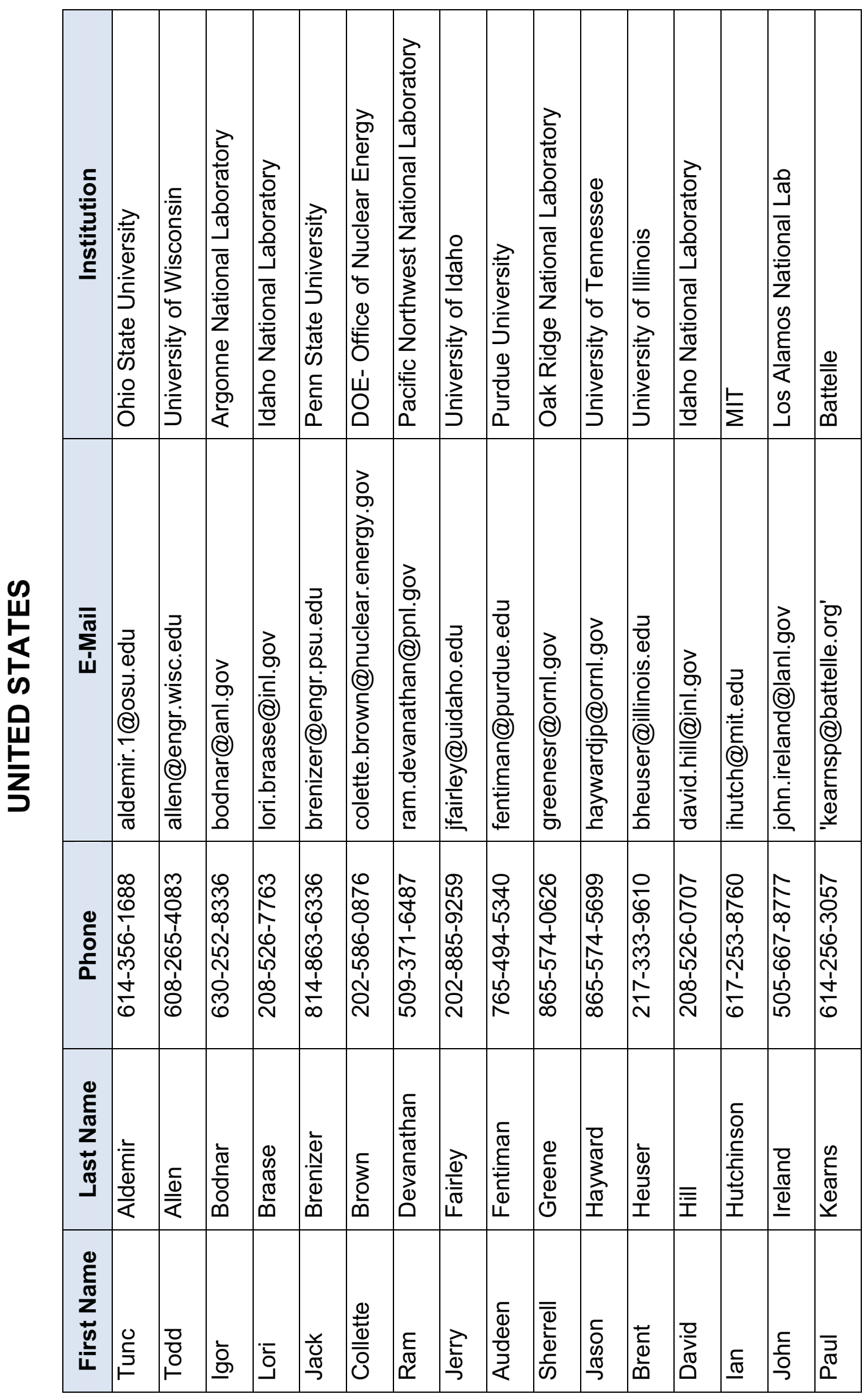


홈

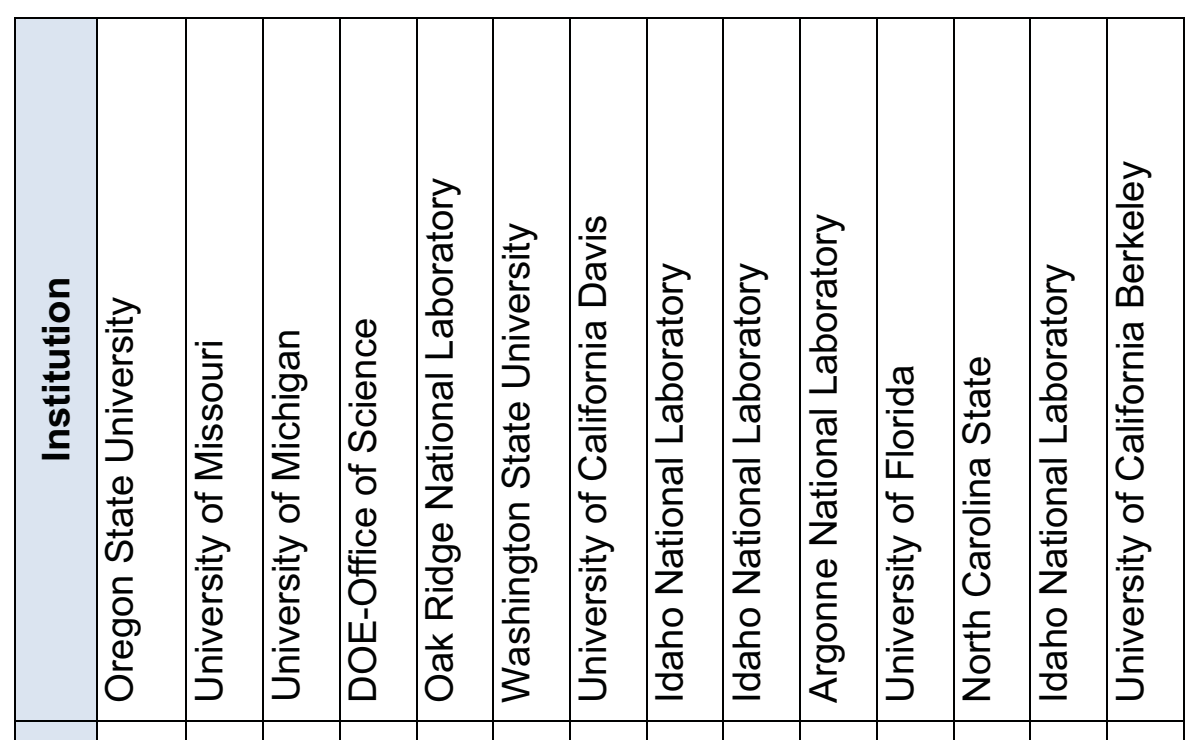

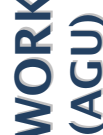

Y.

프르ำ

这

z

So

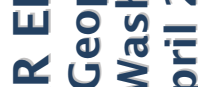

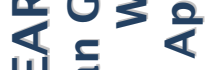

.

容畜

岂

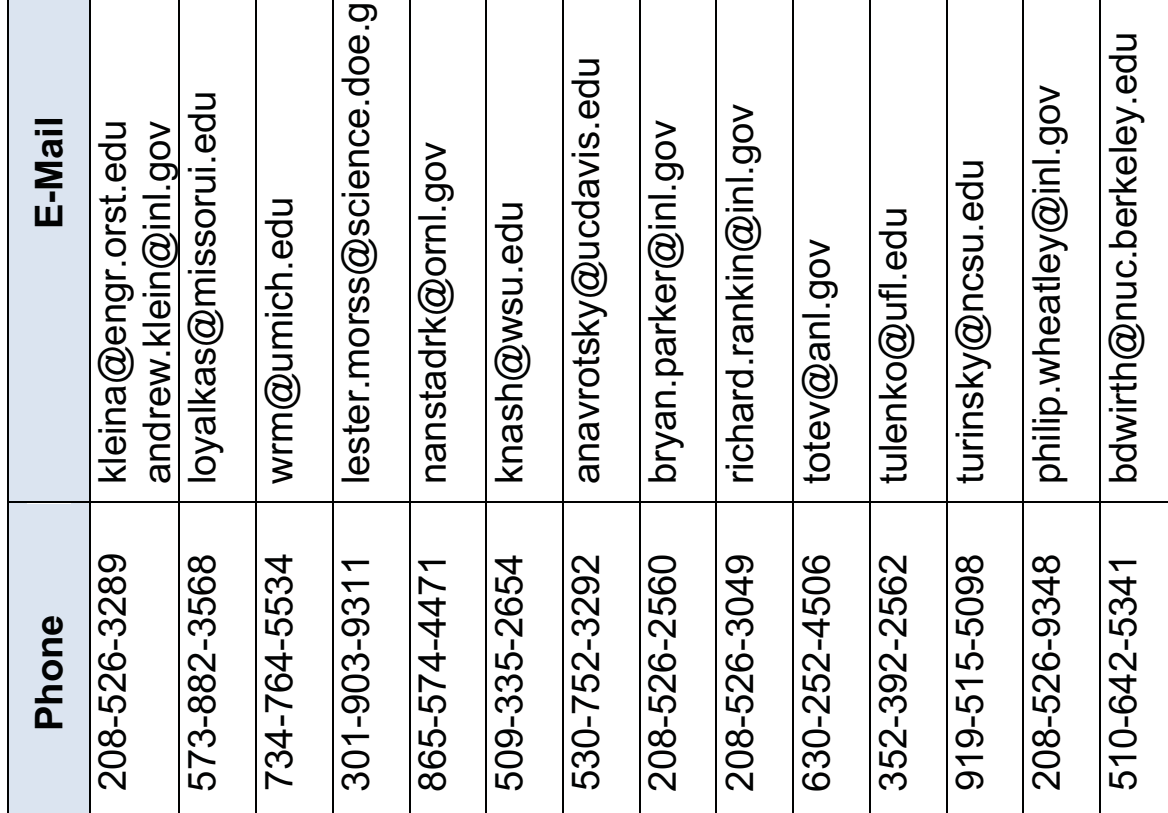

\begin{tabular}{|c|c|c|c|c|c|c|c|c|c|c|c|c|c|c|}
\hline 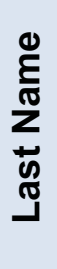 & $\frac{\cdot \frac{c}{\Phi}}{\frac{x}{x}}$ & 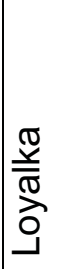 & 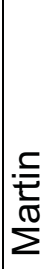 & 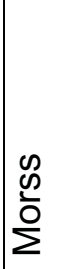 & $\mid \begin{array}{l}\frac{D}{\pi} \\
\frac{\pi}{\omega N} \\
\frac{\pi}{\pi} \\
Z\end{array}$ & $\begin{array}{l}\frac{c}{c} \\
\mathbb{N} \\
Z\end{array}$ & 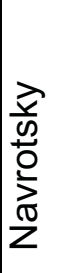 & 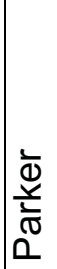 & 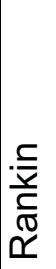 & 文 & 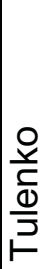 & 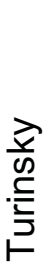 & 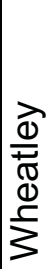 & 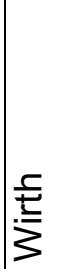 \\
\hline 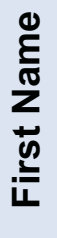 & $\mid \begin{array}{l}\frac{3}{0} \\
\frac{3}{0} \\
\frac{1}{<} \\
\end{array}$ & 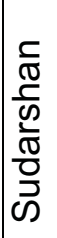 & 点 & 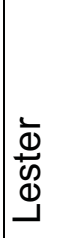 & 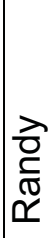 & 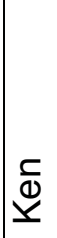 & $\frac{x}{d}$ & 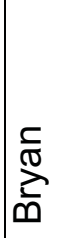 & 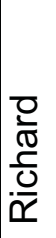 & 帝 & $\frac{\varepsilon}{5}$ & $\begin{array}{l}\overline{\bar{\pi}} \\
\bar{\alpha}\end{array}$ & $\frac{\text { 을 }}{\frac{\bar{a}}{\alpha}}$ & 竞 \\
\hline
\end{tabular}


Appendix B

\section{Attendee Survey Results}



NAME: Paul Howarth

ORGANISATION/INSTITUTION: UK NNL

MY (or my institution's) MAIN AREA(S) OF RESEARCH EXPERTISE:

A special nuclear material management (spent nuclear fuel)

B Fuel technology

I NEED HELP with the following research gap(s) or need(s):

A PU fuel manufacture and burn - up assessment for disposition

B

I CAN (most likely) HELP with the following research gap(s) or need(s):

A Access to PU samples

B PU dedicated facilities for mox fuel manufacture 
NAME: Alex Navrotsky

ORGANISATION/INSTITUTION: UC Davis

MY (or my institution's) MAIN AREA(S) OF RESEARCH EXPERTISE:

A Thermodynamics, calorimetry solid state chemistry

B Geo chemistry, minenalogy

I NEED HELP with the following research gap(s) or need(s):

A Well defined samples for thermochimicro studies atomic scale modelling applicable to new fuels and fuel cycles, waste rad damage

B

I CAN (most likely) HELP with the following research gap(s) or need(s):

A Heats of formation vitrification, sco/nec energies water adsorption energies.

B Correlation to molecules - level stmaturl textures. I have unique high terlerimetric capabilities applications of interest new fuels, e.g nitrides UO2 with RE depants, also THO2 and $\mathrm{CO} 2$ as a $\mathrm{PaO} 2$ analogue ceramic and glass waste forms reaiations damage unanium $\mathrm{V}$ and $\mathrm{VI}$ solids 
NAME: Colin Boxall

ORGANISATION/INSTITUTION: Lancaster University

MY (or my institution's) MAIN AREA(S) OF RESEARCH EXPERTISE:

A Decommissioning robotics. Nuclear instrumentation.

B Tomography of porous metals. Measurement and modelling of ground water flow

I NEED HELP with the following research gap(s) or need(s):

A

$\mathrm{B}$

I CAN (most likely) HELP with the following research gap(s) or need(s):

A Remote NDE via tomography

B 
NAME: Alex Navrotskey

ORGANISATION/INSTITUTION: UC Davis

MY (or my institution's) MAIN AREA(S) OF RESEARCH EXPERTISE:

A Thermodynamics calcinatory solid state chemistry

B

I NEED HELP with the following research gap(s) or need(s):

A Well characterised materials

B Alumistic modelling

I CAN (most likely) HELP with the following research gap(s) or need(s):

A Thermodynamic studies of radiation damage and annision, imperotoes on fuels (also all card in area (1))

B 
NAME: George Smith

ORGANISATION/INSTITUTION: Oxford University

MY (or my institution's) MAIN AREA(S) OF RESEARCH EXPERTISE:

AAtomic scale materials Characterisation (Atomprobe)

B

I NEED HELP with the following research gap(s) or need(s):

A

B

I CAN (most likely) HELP with the following research gap(s) or need(s):

A Study of surveillance sample materials - measure

B Evolution of microstructure, phase chemistry, properties 
NAME: Ayman Hawaii (Paul Turnsky POC)

ORGANISATION/INSTITUTION: NC State University

MY (or my institution's) MAIN AREA(S) OF RESEARCH EXPERTISE:

A Nuclear Data

B

I NEED HELP with the following research gap(s) or need(s):

A

B

I CAN (most likely) HELP with the following research gap(s) or need(s):

A Generation of neutron scattering kernels

B 
NAME: Neil Smart

ORGANISATION/INSTITUTION: NDA

MY (or my institution's) MAIN AREA(S) OF RESEARCH EXPERTISE:

A Geoloqud Disposal

B Waste management

I NEED HELP with the following research gap(s) or need(s):

A Waste form behaviour (SF or Pu or cemetic waste products) long term.

B Aspects of radio include transport in environment

I CAN (most likely) HELP with the following research gap(s) or need(s):

A Exp data and testing for transport model development

B Chemical reaction transport for reactive transport THC modelling 
NAME: Randy Nanstad Sherrell Greene PITBI

ORGANISATION/INSTITUTION: Nuclear Materials Science and Technology Group Oak Ridge National Laboratory

MY (or my institution's) MAIN AREA(S) OF RESEARCH EXPERTISE:

A Materials Science

B Fuel Development/processing

I NEED HELP with the following research gap(s) or need(s):

A Obtain material samples from shutdown reactors to examine for comparisons with test reactor irradiated and with models

B

I CAN (most likely) HELP with the following research gap(s) or need(s):

A Materials examination, and modelling

B irradiation and testing of materials. Radiochemical processing / separations. Instrumentation for NDA (Hayward) 
NAME:

Colin Boxall

ORGANISATION/INSTITUTION:

Lancaster University

MY (or my institution's) MAIN AREA(S) OF RESEARCH EXPERTISE:

A Decommissioning robotics

B Nuclear instrumentation

C Tomography of porous materials

D Measurement and modelling of ground water flow

E Remote sensors and novel analytical methods

I NEED HELP with the following research gap(s) or need(s):

A

B

I CAN (most likely) HELP with the following research gap(s) or need(s):

A $A, B, E, C$ above

B 
NAME:

Belle Upadhyaya (bupadhya@utk.edu)

Wes Hines (jhines2@utk.edu)

ORGANISATION/INSTITUTION:

University of Tennessee

MY (or my institution's) MAIN AREA(S) OF RESEARCH EXPERTISE:

A Monitoring, Reliability, Prognostics

B

I NEED HELP with the following research gap(s) or need(s):

A

B

I CAN (most likely) HELP with the following research gap(s) or need(s):

A Nonradiometic sensors

B 
NAME: Paul Howarth

ORGANISATION/INSTITUTION: UK NNL

MY (or my institution's) MAIN AREA(S) OF RESEARCH EXPERTISE:

A Safe guards / proliferation

B Materials Accountancy

I NEED HELP with the following research gap(s) or need(s):

A Near real time materials accountancy

B proliferation definition (boundary condition)

I CAN (most likely) HELP with the following research gap(s) or need(s):

A Facilitated for PU research. Safe guards techniques

B Materials control. Tracking techniques 
NAME: PI: PITBI

POC: Sandersham Loyalka

ORGANISATION/INSTITUTION: University of NLO - Columbia

MY (or my institution's) MAIN AREA(S) OF RESEARCH EXPERTISE:

A Nuclear safeguards

B Nuclear security

I NEED HELP with the following research gap(s) or need(s):

A

B

I CAN (most likely) HELP with the following research gap(s) or need(s):

A 6c: International activities

B 6d: Public \& Govt confidence 
NAME: Simon Biggs

ORGANISATION/INSTITUTION: Leeds University

MY (or my institution's) MAIN AREA(S) OF RESEARCH EXPERTISE:

A particle manufacture

B Powders handling / mixing for fuel manufacture

I NEED HELP with the following research gap(s) or need(s):

A

B

I CAN (most likely) HELP with the following research gap(s) or need(s):

A

B 
NAME: Todd Allen

ORGANISATION/INSTITUTION: Wisconsin / INL

MY (or my institution's) MAIN AREA(S) OF RESEARCH EXPERTISE:

A Radation damage and transport in fuel

B

I NEED HELP with the following research gap(s) or need(s):

A Post - irradiation exams

B Fuel fabrication

I CAN (most likely) HELP with the following research gap(s) or need(s):

A Reactor testing

B Hot labs/materials archive 
NAME: John Bouchard (POC)

ORGANISATION/INSTITUTION: Open University

MY (or my institution's) MAIN AREA(S) OF RESEARCH EXPERTISE:

A Residual stress and the integrity of welded structures

B High temperature materials performance

I NEED HELP with the following research gap(s) or need(s):

A Local crystal plasticity effects on performance.

B Access to facilities to irradiate and test samples in reactor environments (welded). How to fabricate ODS Materials (welding)

I CAN (most likely) HELP with the following research gap(s) or need(s):

A LWR carch invitation and growth (residual stress related issues)

B predictive physically based models of component lifelines. High temperature components and structures 
NAME: Sudarsham K. Loyalka (also coroning for colleagues at Mission Uni)

ORGANISATION/INSTITUTION: University of Mission

MY (or my institution's) MAIN AREA(S) OF RESEARCH EXPERTISE:

A VHTR's

B Multi physics / multi scale simulations

I NEED HELP with the following research gap(s) or need(s):

A Reactor material performance: Aualysist safety

B Reactor materials performance GEN IV (including VHTR'S)

I CAN (most likely) HELP with the following research gap(s) or need(s):

A Same as above; these are topics where we could help

B 
NAME: Martin Newby PITBI

ORGANISATION/INSTITUTION: City University, London

MY (or my institution's) MAIN AREA(S) OF RESEARCH EXPERTISE:

A Stochastic modelling. Lifecycle models including aging, monitoring and inspection based on evolution of performance characteristics - PRA

B Elicitation of probabilities, uncertainty propagation; stochastic processes; propagation of uncertainty

I NEED HELP with the following research gap(s) or need(s):

A

B

I CAN (most likely) HELP with the following research gap(s) or need(s):

A Uncertainty quantification and validation and verification

B Experimental design/data analysis for monitoring data 
NAME: T Toter

ORGANISATION/INSTITUTION: ANL

MY (or my institution's) MAIN AREA(S) OF RESEARCH EXPERTISE:

A Nuclear fuel performance

B probabilistic models

I NEED HELP with the following research gap(s) or need(s):

A

B

I CAN (most likely) HELP with the following research gap(s) or need(s):

A Coupled codes for FP and thermal - hyoboulog

B 
NAME: Becky Lunn

ORGANISATION/INSTITUTION: University of Strathclyde

MY (or my institution's) MAIN AREA(S) OF RESEARCH EXPERTISE:

A Hydro - mechanical modelling of porous saturated materials (soils/rocks/backfill)

B Crack - sealing using microbes

I NEED HELP with the following research gap(s) or need(s):

A Fast efficient multi - scale modelling

B Multiphase reactive transport models

I CAN (most likely) HELP with the following research gap(s) or need(s):

A Data collection/site characterisation

B Fracture sealing using microbes 
NAME: Larry Miller (ifmiller@utk.edu)

ORGANISATION/INSTITUTION: University of Tennessee

MY (or my institution's) MAIN AREA(S) OF RESEARCH EXPERTISE:

A Safety, security, safeguards

B SNF and Waste management

I NEED HELP with the following research gap(s) or need(s):

A

B

I CAN (most likely) HELP with the following research gap(s) or need(s):

A Uncertainty evaluation related to fuel cycles

B 
NAME:

Colin Boxall

ORGANISATION/INSTITUTION:

Lancaster University

MY (or my institution's) MAIN AREA(S) OF RESEARCH EXPERTISE:

(TRAINING)

A MSc in Decommissioning

MSc in Safety Engineering

BEng/MEng in Nuclear Engineering

B

I NEED HELP with the following research gap(s) or need(s):

A Student exchanges

B

I CAN (most likely) HELP with the following research gap(s) or need(s):

A Integration of construction/engineering skills and dealing with radioactive materials

B 
NAME:

Shenell Greene (poc) PITBI

ORGANISATION/INSTITUTION:

ORNL

MY (or my institution's) MAIN AREA(S) OF RESEARCH EXPERTISE:

A $\quad D: D$

B

I NEED HELP with the following research gap(s) or need(s):

A

B

I CAN (most likely) HELP with the following research gap(s) or need(s):

A Radiochem Cleamp Process Dev

B Robotic Systems

C Sensors 
NAME: Colin Boxall

ORGANISATION/INSTITUTION: Lancaster University

MY (or my institution's) MAIN AREA(S) OF RESEARCH EXPERTISE:

A Decommissioning Robotics. Nuclear Instrumentation

B Tomography of porous materials. Measurement \& modelling of groundwater flow

I NEED HELP with the following research gap(s) or need(s):

A

B)

I CAN (most likely) HELP with the following research gap(s) or need(s):

A High efficiency low lod radiation detectors \& identification capabilities (Nuclear instrumentation)

B 
NAME: PI: Audeen Fentiman and Colleagues

ORGANISATION/INSTITUTION: Purdue University

MY (or my institution's) MAIN AREA(S) OF RESEARCH EXPERTISE:

A Monitoring

B Public \& Government confidence

I NEED HELP with the following research gap(s) or need(s):

A

B

I CAN (most likely) HELP with the following research gap(s) or need(s):

A Monitoring technology development

B Public understanding \& acceptance of nuclear technology 
NAME: Simon Pimblott

ORGANISATION/INSTITUTION: University of Manchester

MY (or my institution's) MAIN AREA(S) OF RESEARCH EXPERTISE:

A Radiation - induced damage

B Simulation and modelling

I NEED HELP with the following research gap(s) or need(s):

A Radiation induced degradation of fuel forms

B

I CAN (most likely) HELP with the following research gap(s) or need(s):

A Radiation damage of ceramics

B Multi scale modelling - atomistic to real scale 
NAME: Sherrell green (POC) PITBI

ORGANISATION/INSTITUTION: ORNL

MY (or my institution's) MAIN AREA(S) OF RESEARCH EXPERTISE:

A Nuclear materials and fuels

B Radiochemical \& reprocessing process development \& SIT

I NEED HELP with the following research gap(s) or need(s):

A

B

I CAN (most likely) HELP with the following research gap(s) or need(s):

A Hot radiochem process development

B matls \& fuels synthesis \& fabrication Irradiation \& PIE. HPC \& simulation 
NAME: Grovenor and Smith

ORGANISATION/INSTITUTION: Oxford University

MY (or my institution's) MAIN AREA(S) OF RESEARCH EXPERTISE:

A Nanoscale characterisation. Structment chemistry with unique range of analytical facilities advanced THE/3D atom probe / nano sims

B Micro scale mechanical testine methologies for surface layers individual grain boundaries including in corrosive environments (Steve Roberts)

I NEED HELP with the following research gap(s) or need(s):

A Really interesting materials to study (1) well characterised irradiated samples. (2) Novel / new materials

B Access to heavy ion irradiation facilites

I CAN (most likely) HELP with the following research gap(s) or need(s):

A Atomic scale characterisation with our suite of instruments and the expertise to use them

B 
NAME: Brent Heuger

ORGANISATION/INSTITUTION: University of Illinois @Urban Champangu

MY (or my institution's) MAIN AREA(S) OF RESEARCH EXPERTISE:

A Micro analytical analysis of materials

B Hydrogen in metals

I NEED HELP with the following research gap(s) or need(s):

A

B

I CAN (most likely) HELP with the following research gap(s) or need(s):

A Thin film UO2 and actinide samples

B Analysis of materials using scattering and other techniques 
NAME: Matthew Eaton

ORGANISATION/INSTITUTION: Imperial College

MY (or my institution's) MAIN AREA(S) OF RESEARCH EXPERTISE:

A Uncertainty Modelling

B Multi scale and multi physics

I NEED HELP with the following research gap(s) or need(s):

A Nuclear data

BUA for non linear system

I CAN (most likely) HELP with the following research gap(s) or need(s):

A Optimisation of fuel cycle (Jon Carter)

B Multi physics and uncerty modelling (Pain and Eaton) 
NAME: Audeen Fentiman (for colleagues)

ORGANISATION/INSTITUTION: Purdue University

MY (or my institution's) MAIN AREA(S) OF RESEARCH EXPERTISE:

A Radiation detection

B Safety and Safeguards. Thermal hydraulics

I NEED HELP with the following research gap(s) or need(s):

A

B

I CAN (most likely) HELP with the following research gap(s) or need(s):

A Modelling and simulation tied to experiments and testing - particularly thermal hydraulics for BWRs

B 
NAME: Richard Shaw (POC)

ORGANISATION/INSTITUTION: British Geological Survey

MY (or my institution's) MAIN AREA(S) OF RESEARCH EXPERTISE:

A Geosciences aspects of deep geological disposal of radioactive waste

B Multi - phase fluid flow in 'geological' media inc low permeability materials

I NEED HELP with the following research gap(s) or need(s):

A

B

I CAN (most likely) HELP with the following research gap(s) or need(s):

A Site characterisation. Proofing access to URL's in Europe

B Experimental data on transport properties through low permeability media (e.g. Bentonites, mudstones)and permeable geological materials 
NAME: James Tulenko

ORGANISATION/INSTITUTION: University of Florida

MY (or my institution's) MAIN AREA(S) OF RESEARCH EXPERTISE:

A SNF and waste disposal

B Thoris behennin repository

I NEED HELP with the following research gap(s) or need(s):

A

B

I CAN (most likely) HELP with the following research gap(s) or need(s):

A Carry out fuel leaching experiment. Fully licensed for Nuclear Fuel

B Carrying out non aqueous reprocessing 
NAME:

Colin Boxall

ORGANISATION/INSTITUTION:

Lancaster University

MY (or my institution's) MAIN AREA(S) OF RESEARCH EXPERTISE:

A Microwave scabbling

B

I NEED HELP with the following research gap(s) or need(s):

A

B

I CAN (most likely) HELP with the following research gap(s) or need(s):

A See above

B 
NAME:

Jerry Fairley

ORGANISATION/INSTITUTION:

University of Idaho

MY (or my institution's) MAIN AREA(S) OF RESEARCH EXPERTISE:

A Multiphase flow \& Heat transport

B Site characterisation (including CFO statistical analysis) for heterogeneous sites

I NEED HELP with the following research gap(s) or need(s):

A Access to data \& sites for testing numerical and conceptual models

B Access to data \& sites for developing \& testing site characterisation methods for complex/heterogeneous sites

I CAN (most likely) HELP with the following research gap(s) or need(s):

A Site characterisations, conceptual model development, sample analysis/planning

B Simulation for uncertainty analysis 
NAME: PI: Andy Klein (for Oregon State)

ORGANISATION/INSTITUTION: Oregon State University

MY (or my institution's) MAIN AREA(S) OF RESEARCH EXPERTISE:

A Radiation detector development POC:Abi Farsoni and David Hamby

B

I NEED HELP with the following research gap(s) or need(s):

A

B

I CAN (most likely) HELP with the following research gap(s) or need(s):

A

B B \& coincidence detection development 
NAME: Martin Newby

ORGANISATION/INSTITUTION: City University (provider Centre for software Reliability)

MY (or my institution's) MAIN AREA(S) OF RESEARCH EXPERTISE:

A Software reliability/safety cases

B

I NEED HELP with the following research gap(s) or need(s):

A

B)

I CAN (most likely) HELP with the following research gap(s) or need(s):

A protection systems/safety cases

B 
NAME: Simon Pimblott (POC) Stuart Lyon and Michael Preuse

ORGANISATION/INSTITUTION: University of Manchester

MY (or my institution's) MAIN AREA(S) OF RESEARCH EXPERTISE:

A Zirconium Oxidation

B Corrosion

I NEED HELP with the following research gap(s) or need(s):

A

B

I CAN (most likely) HELP with the following research gap(s) or need(s):

A Zr oxidation

B 
NAME: James Tulenko (POC)

ORGANISATION/INSTITUTION: University of Florida

MY (or my institution's) MAIN AREA(S) OF RESEARCH EXPERTISE:

A Fuel processing, fabrication, fuel management, fuel performance and fuel disposal - multiscale analysis fuel cycle economics

B

I NEED HELP with the following research gap(s) or need(s):

A Multi scale fuel modelling kinetic Monte Carlo and finite element work to compare with our molecular dynamic work

B

I CAN (most likely) HELP with the following research gap(s) or need(s):

A We have fully luersed fuel processing facilities - provide experimental data

B Expertise in molecular dynamics and extensive computing facility 
NAME: Martin Newby

ORGANISATION/INSTITUTION: City University, London

MY (or my institution's) MAIN AREA(S) OF RESEARCH EXPERTISE:

A Stochastic modelling: typically life cycle models including aging, monitoring and inspection maintenance based on evolution of performance characteristics

B Elicitation of probabilities and uncertainty propagation stochastic process - eg crack growth

I NEED HELP with the following research gap(s) or need(s):

A

B

I CAN (most likely) HELP with the following research gap(s) or need(s):

A Statistical modelling of crack growth based on physical models and environmental / usage factors. Experimental and field data

B Lifetime data analysis. Both classical and based on evolution of iritic charactimsies threshold crossing 
NAME: Bill Martin

ORGANISATION/INSTITUTION: University of Michigan

MY (or my institution's) MAIN AREA(S) OF RESEARCH EXPERTISE:

A Materials effects of radiation (emulate with ion beam deposition) Gary was

B AAV LWR systems - John Lee

I NEED HELP with the following research gap(s) or need(s):

A

B

I CAN (most likely) HELP with the following research gap(s) or need(s):

A Stress couosioncracking

B Exp studies of radiation damage process to test fundamental mechanisms 
NAME: Paul Howarth

ORGANISATION/INSTITUTION: NNL

MY (or my institution's) MAIN AREA(S) OF RESEARCH EXPERTISE:

A Reactor design

B

I NEED HELP with the following research gap(s) or need(s):

A Safety systems assessment (thermal hydrarios, digital C\&l control, passive safe systems) for GEN3 reactions

B Experimental test facilities

I CAN (most likely) HELP with the following research gap(s) or need(s):

A UK assessment technique and data libraries

B From modelling and simulation 
NAME: T Toter

ORGANISATION/INSTITUTION: ANL

MY (or my institution's) MAIN AREA(S) OF RESEARCH EXPERTISE:

A Nuclear fuel performance

B probabilistic models

I NEED HELP with the following research gap(s) or need(s):

A

B

I CAN (most likely) HELP with the following research gap(s) or need(s):

A Sealable tight performance computing

B 
NAME: Richard Taylor

ORGANISATION/INSTITUTION: NNL UK

MY (or my institution's) MAIN AREA(S) OF RESEARCH EXPERTISE:

A Spent fuel disposition

B Fuel Royde

I NEED HELP with the following research gap(s) or need(s):

A Attentive flow sheets for the recycle / conditioning of small quantity / lingh fissile orphan fuels

B

I CAN (most likely) HELP with the following research gap(s) or need(s):

A Active facilities for experiments with snef

B 
NAME: Sandy Birk

ORGANISATION/INSTITUTION: Idaho National Laboratory

MY (or my institution's) MAIN AREA(S) OF RESEARCH EXPERTISE:

A SNF behaviour during storage and disposal

B

I NEED HELP with the following research gap(s) or need(s):

A

B

I CAN (most likely) HELP with the following research gap(s) or need(s):

A Dry cask storage behaviour - SNF degradation during storage - hydride formation treatment

B Repository degradation and critical safety 
NAME:

Steve Donnelly POC

ORGANISATION/INSTITUTION:

University of Salford

MY (or my institution's) MAIN AREA(S) OF RESEARCH EXPERTISE:

A

B

I NEED HELP with the following research gap(s) or need(s):

A

B

I CAN (most likely) HELP with the following research gap(s) or need(s):

A Unused/under-used facilities available for hot expts (UK gap need)

B 


\section{NAME:}

Derek Wadsworth

ORGANISATION/INSTITUTION:

Idaho National Laboratory

MY (or my institution's) MAIN AREA(S) OF RESEARCH EXPERTISE:

A Robotics

B Remote ops/inspection

I NEED HELP with the following research gap(s) or need(s):

A Test/demo sites

B

I CAN (most likely) HELP with the following research gap(s) or need(s):

A Remote operation monitoring devices

B UAV remote $D \& D$ robotics 
NAME: (Ian Hutchinson) PI Jacky Yanch

ORGANISATION/INSTITUTION: MIT

MY (or my institution's) MAIN AREA(S) OF RESEARCH EXPERTISE:

A Radiation transport and interactions

B Low level effects of rediation

I NEED HELP with the following research gap(s) or need(s):

A

B

I CAN (most likely) HELP with the following research gap(s) or need(s):

A Long term effects of low dose radiation

B 


\section{NAME: Ian Farnan POC}

Bill Nuttall PI

ORGANISATION/INSTITUTION: University of Cambridge Engineering (Nuttall)

MY (or my institution's) MAIN AREA(S) OF RESEARCH EXPERTISE:

A Technology policy, Nuclear energy policy and security future systems

B Accelerator driven systems through fuel cycle fusion/fission hybrids

I NEED HELP with the following research gap(s) or need(s):

A

B)

I CAN (most likely) HELP with the following research gap(s) or need(s):

A Economic analysis of future nuclear energy systems

B 
NAME: Simon Pimblott (POC) Ping Xiao (PI)

ORGANISATION/INSTITUTION: University of Manchester

MY (or my institution's) MAIN AREA(S) OF RESEARCH EXPERTISE:

A Triso particles

B High temp oxidation

I NEED HELP with the following research gap(s) or need(s):

A

B

I CAN (most likely) HELP with the following research gap(s) or need(s):

A New and novel fuels and their performance

B 
NAME: Sherrell Greene (POC) PITBI

ORGANISATION/INSTITUTION: ORNL

MY (or my institution's) MAIN AREA(S) OF RESEARCH EXPERTISE:

A Fuel technology

B

I NEED HELP with the following research gap(s) or need(s):

A

B

I CAN (most likely) HELP with the following research gap(s) or need(s):

A Multi scale / multi physics simulation

B Fuel fab irradiation PIE - tried actinide targets - oxide - moltem salt 
NAME: Robin Grimes

ORGANISATION/INSTITUTION: Imperial College, London

MY (or my institution's) MAIN AREA(S) OF RESEARCH EXPERTISE:

A Fundamental radiation damage processes

B Cladding performance models

I NEED HELP with the following research gap(s) or need(s):

A Exp studies of radiation resistant materials (processes of materials that give rise to)

B

I CAN (most likely) HELP with the following research gap(s) or need(s):

A Atomic scale processes from which basic physics processes can be derived

B Modelling of ODS steels. Radiation resistant structural materials 
NAME: (lan Hutchinson) PI Ron Ballinger

ORGANISATION/INSTITUTION: MIT Nucl sci Materials SCI MIT Reactor

MY (or my institution's) MAIN AREA(S) OF RESEARCH EXPERTISE:

A Materials for nuclear reactor application

B

I NEED HELP with the following research gap(s) or need(s):

A

B

I CAN (most likely) HELP with the following research gap(s) or need(s):

A Have in-core high-tempor (to $1600 \mathrm{C}$ ) test facility demonstrated

B Corrosion. Degradation cracking triso fuel integrity analysis 
NAME: Paul Howarth

ORGANISATION/INSTITUTION: NNL

MY (or my institution's) MAIN AREA(S) OF RESEARCH EXPERTISE:

A Reactor physics (Design, operations and monitoring)

B

I NEED HELP with the following research gap(s) or need(s):

A Coupled codes for fuel performance and thermal hydraulic assessment of GEN3 systems

B Experimental facilities (Test loops pod bundle facilities)

I CAN (most likely) HELP with the following research gap(s) or need(s):

A Access to UK codes \& data analysis from modelling simulation

B 
NAME: Andy Klein (for Oregon)

ORGANISATION/INSTITUTION: Oregon State University

MY (or my institution's) MAIN AREA(S) OF RESEARCH EXPERTISE:

A Uncertainty quantification and numerical model additions POC: Todd Palmer

B Reaction scaling and thermal/hydraulic testing POC: Brian Woods and Qiao Wu

I NEED HELP with the following research gap(s) or need(s):

A

B

I CAN (most likely) HELP with the following research gap(s) or need(s):

A High performance computer code department

B System / test facility scaling, integral system testing, separate effects $\mathrm{T} / \mathrm{H}$ facility and tests 
NAME: Richard Taylor

ORGANISATION/INSTITUTION: NNL UK

MY (or my institution's) MAIN AREA(S) OF RESEARCH EXPERTISE:

A SNM Disposition

B

I NEED HELP with the following research gap(s) or need(s):

A Pressurisation mechanisms for PU Powder stored in sealed containment

B

I CAN (most likely) HELP with the following research gap(s) or need(s):

A PU active facilities for experiments

B 
NAME: Phil Wheatley

ORGANISATION/INSTITUTION: Idaho National Laboratory

MY (or my institution's) MAIN AREA(S) OF RESEARCH EXPERTISE:

A Irradiated materials handling examination, measurement and analysis

B

I NEED HELP with the following research gap(s) or need(s):

A

B

I CAN (most likely) HELP with the following research gap(s) or need(s):

A Shielded hot cells at various scales capable of handy small to large size fuel, cladding or irradiated materials coupled with analytical facilities

B 
NAME:

Andy Boston (POC)

ORGANISATION/INSTITUTION:

University of Liverpool

MY (or my institution's) MAIN AREA(S) OF RESEARCH EXPERTISE:

A Radiation instrumentation

B MC modeling

I NEED HELP with the following research gap(s) or need(s):

A

B

I CAN (most likely) HELP with the following research gap(s) or need(s):

A Sensors/detectors

B 
NAME:

Rick Demmer/Don Maiers

ORGANISATION/INSTITUTION:

Idaho National Laboratory

MY (or my institution's) MAIN AREA(S) OF RESEARCH EXPERTISE:

A Decontamination and Decommissioning

B

I NEED HELP with the following research gap(s) or need(s):

A

B

I CAN (most likely) HELP with the following research gap(s) or need(s):

A Scabbling concrete surfaces/cleve D\&D

B 
NAME: Matthew Eaton

ORGANISATION/INSTITUTION: Imperial College

MY (or my institution's) MAIN AREA(S) OF RESEARCH EXPERTISE:

A Crud modelling and clad ballooning

B Multi physics modelling

I NEED HELP with the following research gap(s) or need(s):

A Severe accident phenomenon

B)

I CAN (most likely) HELP with the following research gap(s) or need(s):

A

B 
NAME: jack Brenizen, Kenan Unta, PI's

ORGANISATION/INSTITUTION: Penn State University

MY (or my institution's) MAIN AREA(S) OF RESEARCH EXPERTISE:

A Radiation detection and measurement egp $-n \& 8$

B detects development. Nuclear NDT Methods

I NEED HELP with the following research gap(s) or need(s):

A

B

I CAN (most likely) HELP with the following research gap(s) or need(s):

A Radiation instruments

B Advanced radiometrics for SNR detection 
NAME: Simon Pimblott (POC) Tim Abram (PI)

ORGANISATION/INSTITUTION: University of Manchester

MY (or my institution's) MAIN AREA(S) OF RESEARCH EXPERTISE:

A nuclear fuel systems and technology

B

I NEED HELP with the following research gap(s) or need(s):

A

B

I CAN (most likely) HELP with the following research gap(s) or need(s):

A Ceramic fuels

B Fuel Performance 
NAME: Jason Eapen (Paul TurinskyPOC)

ORGANISATION/INSTITUTION: NC State University

MY (or my institution's) MAIN AREA(S) OF RESEARCH EXPERTISE:

A materials simulation

B

I NEED HELP with the following research gap(s) or need(s):

A Coupling of scales in multi scale simulations of fuels

B

I CAN (most likely) HELP with the following research gap(s) or need(s):

A Large scale simulations for materials science

B 
NAME: David Smith

ORGANISATION/INSTITUTION: University of Bristol - Mechanical Engineering

MY (or my institution's) MAIN AREA(S) OF RESEARCH EXPERTISE:

A High temperature deformation and fracture of steels

B

I NEED HELP with the following research gap(s) or need(s):

A Complementary high temperature material test facilities

B

I CAN (most likely) HELP with the following research gap(s) or need(s):

A Understanding the effects of prior history on deformation and fracture - high temperature components and structures

B 
NAME: Arthur Motta PI

ORGANISATION/INSTITUTION: Penn State University

MY (or my institution's) MAIN AREA(S) OF RESEARCH EXPERTISE:

A Materials damage/esp cladding

B Stress corrosion cracking

I NEED HELP with the following research gap(s) or need(s):

A

B

I CAN (most likely) HELP with the following research gap(s) or need(s):

A See above

B 
NAME: Simon Pimblott

ORGANISATION/INSTITUTION: University of Manchester

MY (or my institution's) MAIN AREA(S) OF RESEARCH EXPERTISE:

A Radiation effects

B Modelling \& simulation

I NEED HELP with the following research gap(s) or need(s):

A

B

I CAN (most likely) HELP with the following research gap(s) or need(s):

A Coolant \& infrastructure for next gen \& adv reactors

B 
NAME: PI: PITBI POC: Sandershan K. Loyalka (also for colleagues)

ORGANISATION/INSTITUTION: University of Nicisown - Columbia

MY (or my institution's) MAIN AREA(S) OF RESEARCH EXPERTISE:

A Nuclear safety

B Water chemistry

I NEED HELP with the following research gap(s) or need(s):

A

B

I CAN (most likely) HELP with the following research gap(s) or need(s):

A Water Chemistry, including the saim

B Nuclear safety (source term) 
NAME: Richard Taylor

ORGANISATION/INSTITUTION: NNL UK

MY (or my institution's) MAIN AREA(S) OF RESEARCH EXPERTISE:

A Spent fuel and SNM Disposition

B

I NEED HELP with the following research gap(s) or need(s):

A Socio economic assessment of the implications of disposition decisions for SNM and fuel

B What criteria to use to justify the selection of a disposition option at a National level

I CAN (most likely) HELP with the following research gap(s) or need(s):

A

B 
NAME: Brent Hellsy

ORGANISATION/INSTITUTION: University of Illinois @ Urbana - Champaign

MY (or my institution's) MAIN AREA(S) OF RESEARCH EXPERTISE:

A Micro analytical analysis of materials

B Hydrogen in metals

I NEED HELP with the following research gap(s) or need(s):

A

B

I CAN (most likely) HELP with the following research gap(s) or need(s):

A Thin film UO2 and activide samples

B Analysis of materials using scattering and other techniques 
NAME:

Graham Fairhall

\section{ORGANISATION/INSTITUTION:}

UK NNL

MY (or my institution's) MAIN AREA(S) OF RESEARCH EXPERTISE:

A Decommissioning toolbox techniques development

B Remote deployment in nuclear environment

I NEED HELP with the following research gap(s) or need(s):

A Non radiometric sensors

B Flexible deployment of dismantling/measurement techniques in cell

I CAN (most likely) HELP with the following research gap(s) or need(s):

A Remote engineering R\&D

B Hot cells / testing facilities 


\begin{tabular}{|l|}
\hline NAME: \\
Mike Carpenter \\
\hline Idaho National Laboratory \\
A Fieldable assay / rod analysis \\
B \\
\hline I NEED HELP with the following research gap(s) or need(s): \\
A Test/ demo sites \\
B \\
\hline \hline I CAN (most likely) HELP with the following research gap(s) or need(s): \\
\hline
\end{tabular}




\section{NAME: Simon Pimblott (POC)}

Simon French (PI)

ORGANISATION/INSTITUTION: University of Manchester

MY (or my institution's) MAIN AREA(S) OF RESEARCH EXPERTISE:

A decision support - decision analysis

B Informatics

\section{NEED HELP with the following research gap(s) or need(s):}

A

B)

I CAN (most likely) HELP with the following research gap(s) or need(s):

A Decision support - decision analysis

B Informatics 
NAME: Ken Nash (Contact) Sue Clark PI

ORGANISATION/INSTITUTION: Washington State University (Chemistry)

MY (or my institution's) MAIN AREA(S) OF RESEARCH EXPERTISE:

A Radio analytical Chemistry

B Activide Chemistry / Environmental conditions

I NEED HELP with the following research gap(s) or need(s):

A

B

I CAN (most likely) HELP with the following research gap(s) or need(s):

A Analytical methods for sensor development

B Radiation instrumentation. Radiological lab facilities 
NAME: Paul Howarth

ORGANISATION/INSTITUTION: UK NNL

MY (or my institution's) MAIN AREA(S) OF RESEARCH EXPERTISE:

A Fuel technology

B

I NEED HELP with the following research gap(s) or need(s):

A High burn - up of mox/UO2 fuel for GEN 3 systems

B Mtr fuel fabrication

I CAN (most likely) HELP with the following research gap(s) or need(s):

A Facilities for MTR fuel manufacture

B PIE Facilities. Code analysis from fuel performance analysis 
NAME: Ivan Maldonado (ivan.maldonado@utk.edu) Art Ruggles (aruggles@utk.edu)

ORGANISATION/INSTITUTION: University of Tennessee Oak Ridge National Lab

MY (or my institution's) MAIN AREA(S) OF RESEARCH EXPERTISE:

A

B

I NEED HELP with the following research gap(s) or need(s):

A

B

I CAN (most likely) HELP with the following research gap(s) or need(s):

A In core fuel management calculations (Maldonado)

B Multi - physics modelling and design of experiments related to thermal hydraulics (Ruggles) 
NAME: David Smith

ORGANISATION/INSTITUTION: University of Bristol - Mechanical Engineering

MY (or my institution's) MAIN AREA(S) OF RESEARCH EXPERTISE:

A Role of residual stress on structural integrity in reactor environments

B

I NEED HELP with the following research gap(s) or need(s):

A Access to facilities to irradiate and test welded samples

B

I CAN (most likely) HELP with the following research gap(s) or need(s):

A Measurement of residual stress in large scale components - radiation resistant structural materials

B 
NAME: K L Musty (Paul Turnsky POC)

ORGANISATION/INSTITUTION: NC State University

MY (or my institution's) MAIN AREA(S) OF RESEARCH EXPERTISE:

A Materials science experimentation of structural (steels) materials and cladding

B (Zirconium)

I NEED HELP with the following research gap(s) or need(s):

A

B

I CAN (most likely) HELP with the following research gap(s) or need(s):

A Development of nano materials used for structures (pressure vessel of LWRs)

B 
NAME: Andrew Sherry (Dominique Laurence Hector)

ORGANISATION/INSTITUTION: University of Manchester

MY (or my institution's) MAIN AREA(S) OF RESEARCH EXPERTISE:

A Thermal Hydraulics coupled solid mechanics

B Muld phase flow

I NEED HELP with the following research gap(s) or need(s):

A calibration

$B$ validation

I CAN (most likely) HELP with the following research gap(s) or need(s):

A Multi physics modelling

B 
NAME: Divitsty Anlstsatov (Paul Turnsky POC)

ORGANISATION/INSTITUTION: NC State University

MY (or my institution's) MAIN AREA(S) OF RESEARCH EXPERTISE:

A Transport theory

B mathematical physics

I NEED HELP with the following research gap(s) or need(s):

A

B

I CAN (most likely) HELP with the following research gap(s) or need(s):

A Lower - higher order solutions of transport equation e.g. quasi - diffusion theory

B Multiphysics coupling 
NAME: Richard Taylor

ORGANISATION/INSTITUTION: NNL UK

MY (or my institution's) MAIN AREA(S) OF RESEARCH EXPERTISE:

A Spent fuel Disposition

B Fuel Recycle

I NEED HELP with the following research gap(s) or need(s):

A Disposal behaviour of commercial spent fuel in repository environments

B CFA's and licensing issues for disposal of SNF. What are the main areas of technical under pinning required

I CAN (most likely) HELP with the following research gap(s) or need(s):

A Active facilities for undertaking disposal / storage experiments and SNF

B 
NAME: Mike Carpenter

ORGANISATION/INSTITUTION: Idaho National laboratory

MY (or my institution's) MAIN AREA(S) OF RESEARCH EXPERTISE:

A Field deployable radiation measurement systems

B

I NEED HELP with the following research gap(s) or need(s):

A

B

I CAN (most likely) HELP with the following research gap(s) or need(s):

A In - situ exam of irradiated mat'ls

B 
NAME:

Francis Livens

\section{ORGANISATION/INSTITUTION:}

Manchester

MY (or my institution's) MAIN AREA(S) OF RESEARCH EXPERTISE:

A Mechanisms of contam/decontam

B Development of functional materials

C Optioneering (B Kelly)

D Robotics/remote characterisation and dismanteling

I NEED HELP with the following research gap(s) or need(s):

A Access to test samples and test locations

B

I CAN (most likely) HELP with the following research gap(s) or need(s):

A Decontam technologies (strippables, electrochem)

B Optioneering 


\section{NAME:}

Audeen Fentiman \& colleague

ORGANISATION/INSTITUTION:

Purdue University

MY (or my institution's) MAIN AREA(S) OF RESEARCH EXPERTISE:

A Waste reduction and management

B Civil engineering and construction engineering management

I NEED HELP with the following research gap(s) or need(s):

A

B

I CAN (most likely) HELP with the following research gap(s) or need(s):

A Construction engineering management for D\&D

B 
NAME: Andy Boston (POC)

ORGANISATION/INSTITUTION: University of Liverpool

MY (or my institution's) MAIN AREA(S) OF RESEARCH EXPERTISE:

A Radiation instrumentation

B MC Modelling

I NEED HELP with the following research gap(s) or need(s):

A Detection supply

B)

I CAN (most likely) HELP with the following research gap(s) or need(s):

A 1) detector capabilities

B 14) lager volume detection 
NAME: John Ireland - POC

ORGANISATION/INSTITUTION: LOS Alamo

MY (or my institution's) MAIN AREA(S) OF RESEARCH EXPERTISE:

A Safety / severity / safeguards - safeguards by design

B Licensing of reprocessing facilities proliferation resistant fuel cycles

I NEED HELP with the following research gap(s) or need(s):

A Licensing of reprocessing facilities

B Proliferation metrics

I CAN (most likely) HELP with the following research gap(s) or need(s):

A Facility Security

B Nuclear material Safeguards 
NAME: Robin Grimes

ORGANISATION/INSTITUTION: Imperial College London

MY (or my institution's) MAIN AREA(S) OF RESEARCH EXPERTISE:

A Atomic scale simulations of fission product behaviour in conventional and advanced fuels

B Development of underpinning physics models that describe the behaviour of fission products in fuel

I NEED HELP with the following research gap(s) or need(s):

A Experimental verification of fission product behaviour

B fuel performance code developers who wish to use new physics based models

I CAN (most likely) HELP with the following research gap(s) or need(s):

A provide physics based models for fuel performance codes

B Provide values for fission product behaviour e.g. activation energies for migration as resolution 
NAME: T Tottie

ORGANISATION/INSTITUTION: ANC

MY (or my institution's) MAIN AREA(S) OF RESEARCH EXPERTISE:

A Nuclear fuel cerocevous

B probaleolostic liodals

I NEED HELP with the following research gap(s) or need(s):

A

B

I CAN (most likely) HELP with the following research gap(s) or need(s):

A Fuel cladding performance

B Understanding of troxidation 
NAME: Colin Taylor (Contact David Smith)

ORGANISATION/INSTITUTION: University of Bristol - Civil Engineering

MY (or my institution's) MAIN AREA(S) OF RESEARCH EXPERTISE:

A Non - linear dynamics, earthquake engineering

B

I NEED HELP with the following research gap(s) or need(s):

A Access to large scale seismic facilities

B

I CAN (most likely) HELP with the following research gap(s) or need(s):

A Predictive, physically - based models of component life times

B 
NAME: Ayman Hanari (Paul Turnsky POC)

ORGANISATION/INSTITUTION: NC State University

MY (or my institution's) MAIN AREA(S) OF RESEARCH EXPERTISE:

A user diagnostic facilities on research reactors in support of materials science

B

I NEED HELP with the following research gap(s) or need(s):

A

B

I CAN (most likely) HELP with the following research gap(s) or need(s):

A Access to ultra cold neution source phase imaging neution radiography, position source, and position sensitive power diffractometer

B 
NAME: Andrew Sherry (Tim Abraham)

ORGANISATION/INSTITUTION: University of Manchester

MY (or my institution's) MAIN AREA(S) OF RESEARCH EXPERTISE:

A Reactor design

B Fuel

I NEED HELP with the following research gap(s) or need(s):

A

B

I CAN (most likely) HELP with the following research gap(s) or need(s):

A Optimisation of Nuclear fuel cycle inc. facility deployment.

B Design of optimum experiments. Models/codes for VHT fuels 
NAME: Bill Martin

ORGANISATION/INSTITUTION: University of Michigan

MY (or my institution's) MAIN AREA(S) OF RESEARCH EXPERTISE:

A Reactive design/analysis/simulation (Tom Downae, John Lee, Bill Martin)

B Transport (Ed Larsen, James Holloway, Bill Martin)

I NEED HELP with the following research gap(s) or need(s):

A

B

I CAN (most likely) HELP with the following research gap(s) or need(s):

A Severe accident research

B Safety systems research 
NAME: Peter Storey

ORGANISATION/INSTITUTION: ND, HSE

MY (or my institution's) MAIN AREA(S) OF RESEARCH EXPERTISE:

A Nuclear safety and security regulator (UK)

B

I NEED HELP with the following research gap(s) or need(s):

A The UK needs to scope the extent of issue (outlined below) and then engage with us National Labs. As a minimum both the UK and US share catalogues of samples available. Ideally there may be scope to facilitate simple transfer and optimise samples held

B

I CAN (most likely) HELP with the following research gap(s) or need(s):

A In service sample archiving current position of such samples needs assessing. Industry) licenses and NDA) need to be engaged and requirements justified; My offer of help is to do this. Then I propose that NNL (Graham Fairhall) is engaged as PSI. the UK needs a strategic approach to sample harvesting that is sustainable

B 
NAME: Ron Klingler 1-208-526-0183

ORGANISATION/INSTITUTION: Idaho National laboratory

MY (or my institution's) MAIN AREA(S) OF RESEARCH EXPERTISE:

A Establish system requirements, define technology readiness levels, develop structured research program to achieve end goals

B

I NEED HELP with the following research gap(s) or need(s):

A

B

I CAN (most likely) HELP with the following research gap(s) or need(s):

A Long term strategic approach

B Public understanding / science based approach 
NAME:

Becky Lunn

ORGANISATION/INSTITUTION:

University of Strathclyde

MY (or my institution's) MAIN AREA(S) OF RESEARCH EXPERTISE:

A Crack sealing using microbial technology

B

I NEED HELP with the following research gap(s) or need(s):

A

B

I CAN (most likely) HELP with the following research gap(s) or need(s):

A

B 
NAME: David Blockley (contact David Smith)

ORGANISATION/INSTITUTION: University of Bristol Civil Engineering

MY (or my institution's) MAIN AREA(S) OF RESEARCH EXPERTISE:

A Systems engineering, management of risk

B

I NEED HELP with the following research gap(s) or need(s):

A Emergency response analysis

B)

I CAN (most likely) HELP with the following research gap(s) or need(s):

A International common framework for risk informed decision making B 
NAME: Robin Gardner (Paul Turnisky POC)

ORGANISATION/INSTITUTION: NC State University

MY (or my institution's) MAIN AREA(S) OF RESEARCH EXPERTISE:

A Monte Carlo modelling of radiation detection problems for purpose of solving inverse problem

B

I NEED HELP with the following research gap(s) or need(s):

A

B

I CAN (most likely) HELP with the following research gap(s) or need(s):

A Detection of strategic materials for safeguards

B 
NAME: Neil Hyatt

ORGANISATION/INSTITUTION: University of Sheffield

MY (or my institution's) MAIN AREA(S) OF RESEARCH EXPERTISE:

A processing and fabrication methods to produce aromic oxide fuel analogues

B

I NEED HELP with the following research gap(s) or need(s):

A thermal conductivity of inert matrix fuels - experimental

measurements and modelling

B

I CAN (most likely) HELP with the following research gap(s) or need(s):

A Well characterised and understood samples for thermodynamic studies

B 
NAME: T Tottie

ORGANISATION/INSTITUTION: ANC

MY (or my institution's) MAIN AREA(S) OF RESEARCH EXPERTISE:

A Nuclear fuel behaviour

B probaleolostic liodals

I NEED HELP with the following research gap(s) or need(s):

A

B

I CAN (most likely) HELP with the following research gap(s) or need(s):

A UC, UN, Uox, UO2, U- UO fuel performance assissment

B 
NAME: Simon Pimblott

ORGANISATION/INSTITUTION: University of Manchester

MY (or my institution's) MAIN AREA(S) OF RESEARCH EXPERTISE:

A Radiation effects

B Modelling and simulation

I NEED HELP with the following research gap(s) or need(s):

A Interfacial properties / performance of materials

B

I CAN (most likely) HELP with the following research gap(s) or need(s):

A Radiation effect

B 
NAME: Todd Allen

ORGANISATION/INSTITUTION: Wisconsin / INL

MY (or my institution's) MAIN AREA(S) OF RESEARCH EXPERTISE:

A Corrosion / stress corrosion cracking

B Radiation damage of fuels and materials

I NEED HELP with the following research gap(s) or need(s):

A Post - irradiation testing

B materials Fabrication

I CAN (most likely) HELP with the following research gap(s) or need(s):

A Irradiation testing (ATR and ION beams) and examination (hot labs)

B Corrosion (CO2, water sodium, molten salt) 
NAME: David Smith

ORGANISATION/INSTITUTION: University of Bristol

MY (or my institution's) MAIN AREA(S) OF RESEARCH EXPERTISE:

A Structural integrity

B

I NEED HELP with the following research gap(s) or need(s):

A Uncertainty quantification for highly non-linear systems

B

I CAN (most likely) HELP with the following research gap(s) or need(s):

A Understanding effects of prior loading an structural integrity

B 
NAME: Hany Abdel - khalik (Paul Turinsky POC)

ORGANISATION/INSTITUTION: NC State University

MY (or my institution's) MAIN AREA(S) OF RESEARCH EXPERTISE:

$A \vee \& V, U Q$ and data assimilation

B

I NEED HELP with the following research gap(s) or need(s):

A

B

I CAN (most likely) HELP with the following research gap(s) or need(s):

A UQ \& data assimilation for line sizable problems

B 
NAME: Simon Pimblott

ORGANISATION/INSTITUTION: University of Manchester

MY (or my institution's) MAIN AREA(S) OF RESEARCH EXPERTISE:

A Radiation Effects

B Modelling and simulation

I NEED HELP with the following research gap(s) or need(s):

A New reprocessing systems. Waste form performance

B heterogeneous porous materials

I CAN (most likely) HELP with the following research gap(s) or need(s):

A Effects of B/8 vs \& Vs in irradiation

B Encapsulants for reactive metals 
NAME: John Ireland - Gordon Jarrinen (PoC)

ORGANISATION/INSTITUTION: Los Alamos

MY (or my institution's) MAIN AREA(S) OF RESEARCH EXPERTISE:

A SNF and waste management

B

I NEED HELP with the following research gap(s) or need(s):

A Technitive waste forms

B

I CAN (most likely) HELP with the following research gap(s) or need(s):

A

B 
NAME:

Andy Boston

ORGANISATION/INSTITUTION:

University of Liverpool

MY (or my institution's) MAIN AREA(S) OF RESEARCH EXPERTISE:

A Laser Scabbling

B 2D/5D optics / image overlaying

I NEED HELP with the following research gap(s) or need(s):

A Available test site

B

I CAN (most likely) HELP with the following research gap(s) or need(s):

A Scabbling (concrete) UK

B 
NAME: Simon Pimblott

ORGANISATION/INSTITUTION: University of Manchester

MY (or my institution's) MAIN AREA(S) OF RESEARCH EXPERTISE:

A Radiation Effects

B Modelling \& Simulation

I NEED HELP with the following research gap(s) or need(s):

A Low dose rate effects inferred from high dose rate studies (Risk modelling)

B)

I CAN (most likely) HELP with the following research gap(s) or need(s):

A

B 
NAME: Bill Martin

ORGANISATION/INSTITUTION: University of Michigan

MY (or my institution's) MAIN AREA(S) OF RESEARCH EXPERTISE:

A Risk analysis - static/dynamic (John Lee)

B Advanced safty simulation methods (Tom Downar)

I NEED HELP with the following research gap(s) or need(s):

A

B

I CAN (most likely) HELP with the following research gap(s) or need(s):

A Model US capability for server accidents

B Ajv radiometrics for SNM detection 
NAME: Andrew Sherry (Joe Robson)

ORGANISATION/INSTITUTION: University of Manchester

MY (or my institution's) MAIN AREA(S) OF RESEARCH EXPERTISE:

A Micro structural modelling

B

I NEED HELP with the following research gap(s) or need(s):

A

B

I CAN (most likely) HELP with the following research gap(s) or need(s):

A Kinetic models of micro structural evolution

B 
NAME: Steve Donnelly POC

ORGANISATION/INSTITUTION: University of Salford

MY (or my institution's) MAIN AREA(S) OF RESEARCH EXPERTISE:

A Radiation damage processes in situ TEM oberservation of radiation damage processes

B Inert gases in solids

I NEED HELP with the following research gap(s) or need(s):

A

B

I CAN (most likely) HELP with the following research gap(s) or need(s):

A He behaviour (UK gap/need)

B 
NAME: T Tottie

ORGANISATION/INSTITUTION: ANC

MY (or my institution's) MAIN AREA(S) OF RESEARCH EXPERTISE:

A Nuclear fuel behaviour

B probaleolostic liodals

I NEED HELP with the following research gap(s) or need(s):

A

B

I CAN (most likely) HELP with the following research gap(s) or need(s):

A Fuel performance codes

B 
NAME: Brian Wirth

ORGANISATION/INSTITUTION: UC Berkeley, Department of Nuclear Engineering

MY (or my institution's) MAIN AREA(S) OF RESEARCH EXPERTISE:

A irradiation effects in nuclear materials - computational models and experimental characterisation

B Nuclear fuel performance

I NEED HELP with the following research gap(s) or need(s):

A Providing a library of irradiation structural materials for characterisation of embrittlement/ microstructure

B Computational physicists interested in developing computational methods for long time evolution

I CAN (most likely) HELP with the following research gap(s) or need(s):

A Position annihilation spectroscopy characterisation of irradiated materials

B Kinetic models of defect/solute transport in irradiated materials 
NAME: Andrew Sherry (Tim Abraham)

ORGANISATION/INSTITUTION: University of Manchester

MY (or my institution's) MAIN AREA(S) OF RESEARCH EXPERTISE:

A Reactor design

B Fuel

I NEED HELP with the following research gap(s) or need(s):

A

B

I CAN (most likely) HELP with the following research gap(s) or need(s):

A TH Fuel cycle

B 
NAME: Paul Turinsky

ORGANISATION/INSTITUTION: NC State University

MY (or my institution's) MAIN AREA(S) OF RESEARCH EXPERTISE:

$A \vee \& V, U Q$

B Multiphysics modelling of Nuclear systems

I NEED HELP with the following research gap(s) or need(s):

A UQ for highly nonlinear systems where stochastic sampling is computationally prohibitive generic approach to multi scale modelling for phase space decomposition approaches

B

I CAN (most likely) HELP with the following research gap(s) or need(s):

A UQ and data assimilation for problems with both large data fields and large number of observables

B Multiphysics modelling 
NAME: Simon Pimblott

ORGANISATION/INSTITUTION: University of Manchester

MY (or my institution's) MAIN AREA(S) OF RESEARCH EXPERTISE:

A Radiation Effects

B Modelling and simulation

I NEED HELP with the following research gap(s) or need(s):

A Interfacial (radn-induce) processes of oxides and polymers @ temp \& pressure

B In situ (i.e. under Radn, temp \& pres) examination of materials

I CAN (most likely) HELP with the following research gap(s) or need(s):

A Electronic excitation in models

B Non - aqueous processing technology. Ion beam irradiation 
NAME: Jay Roach

ORGANISATION/INSTITUTION: Idaho National Laboratory

MY (or my institution's) MAIN AREA(S) OF RESEARCH EXPERTISE:

A Pilot scale / demonstration

B

I NEED HELP with the following research gap(s) or need(s):

A

B

I CAN (most likely) HELP with the following research gap(s) or need(s):

A Existing pilot scale cold crucible induction melter with state of the art off gas treatment and research facility

B Steam reformer fluidized bed pilot scale facility 


\section{NAME:}

Richard Shaw (POC)

\section{ORGANISATION/INSTITUTION:}

British Geological Survey

MY (or my institution's) MAIN AREA(S) OF RESEARCH EXPERTISE:

A Public understanding in radwaste context (Julie West - PI)

B Remote sensing techniques (PITBI)

\section{NEED HELP with the following research gap(s) or need(s):}

A

B

I CAN (most likely) HELP with the following research gap(s) or need(s):

A Development of public understanding educational techniques

B Characterisation of contaminated land (non-invasive) 
NAME: Colin Boxall

ORGANISATION/INSTITUTION: Lancaster University

MY (or my institution's) MAIN AREA(S) OF RESEARCH EXPERTISE:

A Decommissioning robotics. Nuclear instrumentation. Tomography of porous materials

B Measurement and modelling of groundwater flow. Elected chemical sensors

I NEED HELP with the following research gap(s) or need(s):

A

B

I CAN (most likely) HELP with the following research gap(s) or need(s):

A Sensor development for high T/corrosive environments

B 
NAME: Jason Hayward haywardjp@ornl.gov

ORGANISATION/INSTITUTION: Oak Ridge national Lab University of Tennessee

MY (or my institution's) MAIN AREA(S) OF RESEARCH EXPERTISE:

A Safety, security and safeguards

B

I NEED HELP with the following research gap(s) or need(s):

A

B

I CAN (most likely) HELP with the following research gap(s) or need(s):

A Data fusion (video/visible light + radiometrics $(\&, N S)+$ inertial)

B 
NAME: lan Farnan POC Bill Nuttall / Geoff Parks PI's

ORGANISATION/INSTITUTION: University of Cambridge Engineering

MY (or my institution's) MAIN AREA(S) OF RESEARCH EXPERTISE:

A In coke optimisation

B ADS and Thorium fuel cycle

I NEED HELP with the following research gap(s) or need(s):

A

B

I CAN (most likely) HELP with the following research gap(s) or need(s):

A Economic and technological assessments of ADS and thorium fuel cycle

B 
NAME: T Tottie

ORGANISATION/INSTITUTION: ANC

MY (or my institution's) MAIN AREA(S) OF RESEARCH EXPERTISE:

A Nuclear fuel behaviour

B probaleolostic liodals

I NEED HELP with the following research gap(s) or need(s):

A

B

I CAN (most likely) HELP with the following research gap(s) or need(s):

A Experimental verification of fission product behaviour

B 
NAME: Andrew Sherry

ORGANISATION/INSTITUTION: University of Manchester

MY (or my institution's) MAIN AREA(S) OF RESEARCH EXPERTISE:

A Materials performance (corrosion / SCC testing structural integrity, meso - scale modelling)

B Materials characterisation (ATEM, FIB, scm tomography $-\mathrm{x}$ ray and synitrotion, residual stress)

I NEED HELP with the following research gap(s) or need(s):

A Testing of irradiated materials - mechanical and fracture toughness

B Testing of irradiated materials SCC

I CAN (most likely) HELP with the following research gap(s) or need(s):

A LWR crack initiation and growth (imaging antoclare facility)

B predictive physically - based models of component / materials lifetime 
NAME: Ram Devanathan

ORGANISATION/INSTITUTION: PNNL

MY (or my institution's) MAIN AREA(S) OF RESEARCH EXPERTISE:

A Modelling fission reactor materials for lifetime extension

B POCs (fei gao Chuck Henager) PNNL

I NEED HELP with the following research gap(s) or need(s):

A Long time scale simulation

B

I CAN (most likely) HELP with the following research gap(s) or need(s):

A Modelling reactor structural materials

B 
NAME: John May

ORGANISATION/INSTITUTION: University of Bristol Civil Engineering

MY (or my institution's) MAIN AREA(S) OF RESEARCH EXPERTISE:

A Safety systems research, statistical testing

B

I NEED HELP with the following research gap(s) or need(s):

A Dealing with obsolescence in computer systems and instrumentation B Uncertainty quantification

I CAN (most likely) HELP with the following research gap(s) or need(s):

A Design of optimum experiments

B 
NAME: Sevnggin Kim Fan Bill Cheung PI

ORGANISATION/INSTITUTION: Penn State University

MY (or my institution's) MAIN AREA(S) OF RESEARCH EXPERTISE:

A Severe accident modelling and eapmiments

B

I NEED HELP with the following research gap(s) or need(s):

A

B

I CAN (most likely) HELP with the following research gap(s) or need(s):

A Running $\mathrm{T} / \mathrm{H}$ experiments in nod bundle facility

B 
NAME: Colin Boxall

ORGANISATION/INSTITUTION: Lancaster University

MY (or my institution's) MAIN AREA(S) OF RESEARCH EXPERTISE:

A Novel analytical methods and sensors. Novel membrane materials for separations and dialysis / electro osmosis

B Urex and novel reprocessing routes. Chemical / hydro dynamic kinetic modelling

I NEED HELP with the following research gap(s) or need(s):

A Hot labs for Urex / novel reprocessing data

B

I CAN (most likely) HELP with the following research gap(s) or need(s):

A

B 
NAME: Sanderson Layalka

ORGANISATION/INSTITUTION: University of NM Columbia

MY (or my institution's) MAIN AREA(S) OF RESEARCH EXPERTISE:

A Direct energy conversion sensors/detectors

B

I NEED HELP with the following research gap(s) or need(s):

A $4 \mathrm{~h}$ ? utilization of nuclear waste

B

I CAN (most likely) HELP with the following research gap(s) or need(s):

A 4d disposal

B 
NAME: Roser Smith (PI John Andrews : J.D.Andrews@llboro.ac.uk)

ORGANISATION/INSTITUTION: Loughborough University

MY (or my institution's) MAIN AREA(S) OF RESEARCH EXPERTISE:

A Risk and Reliability Modelling - quantitative

B

I NEED HELP with the following research gap(s) or need(s):

A

B

I CAN (most likely) HELP with the following research gap(s) or need(s):

A Quantitative risk assessment

$B$ 
NAME: Wes Hines (jhinesz@utk.edu) Jason Hayward (jhayward@utk.edu)

ORGANISATION/INSTITUTION: University of Tennessee

MY (or my institution's) MAIN AREA(S) OF RESEARCH EXPERTISE:

A Online monitoring and safeguards

B Data validation and Processing

I NEED HELP with the following research gap(s) or need(s):

A Indentifying facility needs

B Identifying key measurement points

I CAN (most likely) HELP with the following research gap(s) or need(s):

A Near real time material accountancy

B Risk analysis. Sensor/system modeling 
NAME: Ian Farnan POC PI

ORGANISATION/INSTITUTION: University of Cambridge Earth Sciences

MY (or my institution's) MAIN AREA(S) OF RESEARCH EXPERTISE:

A Local structure in nuclear fuels - inert matrix, UO2/UO2 + X TRISO Fuels

B He incorporation in UO2 fission product incorporation UO2 e.g. CS

I NEED HELP with the following research gap(s) or need(s):

A Sample production, characterisation and shipping

B

I CAN (most likely) HELP with the following research gap(s) or need(s):

A Element special spectroscopy: - nuclear magnetic resourance masnmr on radiological samples

B 
NAME: T Tottie

ORGANISATION/INSTITUTION: ANC

MY (or my institution's) MAIN AREA(S) OF RESEARCH EXPERTISE:

A Nuclear fuel behaviour

B probaleolostic liodals

I NEED HELP with the following research gap(s) or need(s):

A PIE

B

I CAN (most likely) HELP with the following research gap(s) or need(s):

A Hog curuup studies

B 
NAME: Steve Donelly POC

ORGANISATION/INSTITUTION: University of Salford

MY (or my institution's) MAIN AREA(S) OF RESEARCH EXPERTISE:

A Radiation damage processes - in-situ TEM obersavation of radiation damage processes

B Smart gases in solids

I NEED HELP with the following research gap(s) or need(s):

A

$B$

I CAN (most likely) HELP with the following research gap(s) or need(s):

A Experimental studies of radiation damage processes to test.

B Fundamental mechanisms. Effects of HE on materials properties (UK gap/Need) 
NAME: John Ireland - Stewart Maloy (POC)

ORGANISATION/INSTITUTION: Los Alamos

MY (or my institution's) MAIN AREA(S) OF RESEARCH EXPERTISE:

A Reactor materials performance

B

I NEED HELP with the following research gap(s) or need(s):

A Data

B

I CAN (most likely) HELP with the following research gap(s) or need(s):

A Hot coals PIE

B 
NAME: Paul Smith

ORGANISATION/INSTITUTION: Loughborough University

MY (or my institution's) MAIN AREA(S) OF RESEARCH EXPERTISE:

A Computational - modelling - molecular dynamics, ab-initio - linked md- finite elements

B Continuum modelling: heat transfer (etc) ceramics/metals/covalent systems

I NEED HELP with the following research gap(s) or need(s):

A Developing multi component potentials for nuclear materials

B Efficient saddle - point finding algorithms for long time dynamics

I CAN (most likely) HELP with the following research gap(s) or need(s):

A linking finite elements to molecular dynamics

B Modelling radiation events 
NAME: (Point of contact lan Hutchinson) PI Jacopo Buongiosho

ORGANISATION/INSTITUTION: MIT

MY (or my institution's) MAIN AREA(S) OF RESEARCH EXPERTISE:

A Thermal Hydraulics. Critical heat flux experiments. Reactor engineering

B Nanofluid characterization and nuclear energy applications

I NEED HELP with the following research gap(s) or need(s):

A Multifluid computational fluid dynamics and modelling

B

I CAN (most likely) HELP with the following research gap(s) or need(s):

A Reactor thermal engineering and physics

B 
NAME: Francis Livens

ORGANISATION/INSTITUTION: University of Manchester

MY (or my institution's) MAIN AREA(S) OF RESEARCH EXPERTISE:

A Radioelement chemistry and geochemistry

B Waste form performance (with Sheffield). Separation chemistry

I NEED HELP with the following research gap(s) or need(s):

A Transport expts, especially inert atmosphere and transoranics

B Prep and characterisation of highly active materials

I CAN (most likely) HELP with the following research gap(s) or need(s):

A Coupled thermodynamic / kinetic speciation modelling

B 
NAME: Bill Martin

ORGANISATION/INSTITUTION: University of Michigan

MY (or my institution's) MAIN AREA(S) OF RESEARCH EXPERTISE:

A Waste forms - Lumin Wang - Rod Ewing

B Adv fuel cycles - John Lee (modelling)

I NEED HELP with the following research gap(s) or need(s):

A

B

I CAN (most likely) HELP with the following research gap(s) or need(s):

A Adv fuel cycle analysis

B Pu disposition / encapsulation 
NAME: Andy Boston (PETBE)

ORGANISATION/INSTITUTION: University of Liverpool

MY (or my institution's) MAIN AREA(S) OF RESEARCH EXPERTISE:

A Laser scrabbling

B 2D/3D optics image overlay

I NEED HELP with the following research gap(s) or need(s):

A

B

I CAN (most likely) HELP with the following research gap(s) or need(s):

A Remote technologies

B Data fusion to combine multiple sensors (2) 
NAME: Sherrell Greene POC

ORGANISATION/INSTITUTION: ORNL

MY (or my institution's) MAIN AREA(S) OF RESEARCH EXPERTISE:

A Safety

B

I NEED HELP with the following research gap(s) or need(s):

A

B

I CAN (most likely) HELP with the following research gap(s) or need(s):

A LWR,GcR,SFR modelling \& sim

B Wireless cyber security 
NAME: Grovenor and Smith

ORGANISATION/INSTITUTION: Oxford University

MY (or my institution's) MAIN AREA(S) OF RESEARCH EXPERTISE:

A nanoscale characterization, structure and chemistry with unique range of analytical facilities

B

I NEED HELP with the following research gap(s) or need(s):

A Really interesting materials to study

B

I CAN (most likely) HELP with the following research gap(s) or need(s):

A Atomic scale characterization

B 
NAME: Brent Heuger

ORGANISATION/INSTITUTION: University of Illinois @ Urbana - Champargu

MY (or my institution's) MAIN AREA(S) OF RESEARCH EXPERTISE:

A Micro analytical analysis of materials

B hydrogen in metals

I NEED HELP with the following research gap(s) or need(s):

A

B

I CAN (most likely) HELP with the following research gap(s) or need(s):

A Thin film UO2 actinide samples

B Analysis of materials using scattering of other techniques 
NAME: Roser Smith

ORGANISATION/INSTITUTION: Loughborough University

MY (or my institution's) MAIN AREA(S) OF RESEARCH EXPERTISE:

A Radiation resistance from a theoretical view point

B Long time materials performance - theoretical

I NEED HELP with the following research gap(s) or need(s):

A

B

I CAN (most likely) HELP with the following research gap(s) or need(s):

A Irradiation resistance predictive modelling

B Multi scale modelling 
NAME: Randy Nanstad PITBI

ORGANISATION/INSTITUTION: Nuclear materials science and technology group materials science and technology division Oak Ridge National Laboratory

MY (or my institution's) MAIN AREA(S) OF RESEARCH EXPERTISE:

A Structural materials wide range of materials science capabilities, including test reactor (HFIR), hot cells microscopes, etc

B Modelling and micro structural expertise atomistic M.D., kinetic multiscale for structural, rad. Effects, fuels etc

I NEED HELP with the following research gap(s) or need(s):

A IASCC experiments and modelling

B Data and materials for examination at high fluence / both low and hi flux to facilitate modelling prediction of RPU embrittlement to soy

I CAN (most likely) HELP with the following research gap(s) or need(s):

A irradiation and testing irrad. Matls, high temperature test facilities

B Link mechanistic modelling, theory with experimental. Modelling simulation. 
NAME: Paul Wilson

ORGANISATION/INSTITUTION: Wisconsin

MY (or my institution's) MAIN AREA(S) OF RESEARCH EXPERTISE:

A Reactor Physics

B Fuel cycle analysis

I NEED HELP with the following research gap(s) or need(s):

A

B

I CAN (most likely) HELP with the following research gap(s) or need(s):

A Fuel cycle analysis

B 
NAME: Steve Donnelly

ORGANISATION/INSTITUTION: University of Salford

MY (or my institution's) MAIN AREA(S) OF RESEARCH EXPERTISE:

A

B

I NEED HELP with the following research gap(s) or need(s):

A

B

I CAN (most likely) HELP with the following research gap(s) or need(s):

A unused / under used hot facilities (UK gap/deed)

B 
NAME: A Navrotsky

ORGANISATION/INSTITUTION: UC Davis

MY (or my institution's) MAIN AREA(S) OF RESEARCH EXPERTISE:

A Thermodynamic cabizinatys solid state chemistry

B Adv fuel cycles - John Lee (modelling)

I NEED HELP with the following research gap(s) or need(s):

A Samples

B Atomistic modelling

I CAN (most likely) HELP with the following research gap(s) or need(s):

A Thermodynamic of waste forms, elements inenfonaton ned damage also si cardin area 1

B 
NAME: Graham Fairham

ORGANISATION/INSTITUTION: UKNNL

MY (or my institution's) MAIN AREA(S) OF RESEARCH EXPERTISE:

A Environmental risk assessment approach

B Fixation of radioactive species in cad/buildings

I NEED HELP with the following research gap(s) or need(s):

A Consistent approach to defining risk based end points B Remediation techniques (in Situ)

I CAN (most likely) HELP with the following research gap(s) or need(s):

A UK methodology for contaminated land BPEO

B Modelling of radioactive species transport e.g. TC, SR 
NAME: Jason Hayward (jhayward@utk.edu)

ORGANISATION/INSTITUTION: University of Tennessee oak Ridge national Lab

MY (or my institution's) MAIN AREA(S) OF RESEARCH EXPERTISE:

A Safety security and safeguards

B

I NEED HELP with the following research gap(s) or need(s):

A Identify high priority needs for nuclear data relevant to safeguards and security

B

I CAN (most likely) HELP with the following research gap(s) or need(s):

A Design of detectors and images for nuclear security and safeguards/ advanced radiometrics for SNM detection

B 
NAME: Bill Martin

ORGANISATION/INSTITUTION: University of Michigan

MY (or my institution's) MAIN AREA(S) OF RESEARCH EXPERTISE:

A Radiation damage to cladding and structural materials (WAS)

B Fuel Performance modelling (WAS)

I NEED HELP with the following research gap(s) or need(s):

A

B

I CAN (most likely) HELP with the following research gap(s) or need(s):

A Thermodynamic phase charge modelling

B Fuel cladding perf in RX env 
NAME: Audeen Fentiman (For Colleagues)

ORGANISATION/INSTITUTION: Purdue University

MY (or my institution's) MAIN AREA(S) OF RESEARCH EXPERTISE:

A Materials performance under extreme conditions

B Construction engineering management

I NEED HELP with the following research gap(s) or need(s):

A

B

I CAN (most likely) HELP with the following research gap(s) or need(s):

A Materials development and testing

B Modelling and simulation 
NAME: Sherrell Greene

ORGANISATION/INSTITUTION: ORNL

MY (or my institution's) MAIN AREA(S) OF RESEARCH EXPERTISE:

A Reactor design ops

B

I NEED HELP with the following research gap(s) or need(s):

A

B

I CAN (most likely) HELP with the following research gap(s) or need(s):

A MSR, AHTR, GCR concept dev. liC? Diagnostics.

B Small LWR dev. Thermal hydraulics 
NAME: Simon Biggs

ORGANISATION/INSTITUTION: Leeds University

MY (or my institution's) MAIN AREA(S) OF RESEARCH EXPERTISE:

A long term behaviour of cements and grants (PI: lan Richardson)

B

I NEED HELP with the following research gap(s) or need(s):

A

B

I CAN (most likely) HELP with the following research gap(s) or need(s):

A unused / under used hot facilities (UK gap/deed)

B 
NAME: Ram Devanathan

ORGANISATION/INSTITUTION: Pacific Northwest national Lab (PNNL)

MY (or my institution's) MAIN AREA(S) OF RESEARCH EXPERTISE:

A Waste forms(experiment and modelling) Dennis Strachan(PNNL) John Vienna (PNNL)

B Modelling of fuels and structural materials Ram Devanathan

I NEED HELP with the following research gap(s) or need(s):

A bridging the scales of the problem - going from atomic level to macroscopic level

B Develop methods to include electronic excitation effects in radiation damage models

I CAN (most likely) HELP with the following research gap(s) or need(s):

A Ion implantation and analysis facilities POC: Dr Yanwen Zhang yanwen.zhang@pnl.gov

B Inter atomic potential development for multi-component systems POC: Ram Devanathan ram.devanathan@pnl.gov Experimental studies of waste forms POC Dennis Strachan 
NAME: Becky Lunn

ORGANISATION/INSTITUTION: University of Strathclyde

MY (or my institution's) MAIN AREA(S) OF RESEARCH EXPERTISE:

A Public perception of risk and uncertainty (to date using examples from flooding and climate change)

B

I NEED HELP with the following research gap(s) or need(s):

A Appling methodology to nuclear engineering including waste management and disposal

B

I CAN (most likely) HELP with the following research gap(s) or need(s):

A public perception of risk/decision making a based on uncertain science

$\mathrm{B}$ 
NAME: Jerry Fairley

ORGANISATION/INSTITUTION: University of Idaho

MY (or my institution's) MAIN AREA(S) OF RESEARCH EXPERTISE:

A Multi phase flow and heat transport modelling

B Site characterisation of heterogeneous sites modelling and characterisation of thermally-driven systems

I NEED HELP with the following research gap(s) or need(s):

A Instrumentation and sensor development for monitoring in high temperatures and /or corrosive environments B

I CAN (most likely) HELP with the following research gap(s) or need(s):

A Field support and input for sensor design

B 
NAME: John Ireland - Ken McClellen (POC)

ORGANISATION/INSTITUTION: Los Alamos

MY (or my institution's) MAIN AREA(S) OF RESEARCH EXPERTISE:

A Fuel technology - oxides and Pu bard fuels

B Fuel performance codes

I NEED HELP with the following research gap(s) or need(s):

A Data

B

I CAN (most likely) HELP with the following research gap(s) or need(s):

A High performance computing - cetin, Unal, (POC)

B 
NAME: Brent Heusen representing colleagues

ORGANISATION/INSTITUTION: University of Illinois @ Urkana - champongy

MY (or my institution's) MAIN AREA(S) OF RESEARCH EXPERTISE:

A Neutronics \& thermohydraulics modelling

B

I NEED HELP with the following research gap(s) or need(s):

A

B

I CAN (most likely) HELP with the following research gap(s) or need(s):

A

B 
NAME: Simon Biggs (POC)

ORGANISATION/INSTITUTION: Leeds University

MY (or my institution's) MAIN AREA(S) OF RESEARCH EXPERTISE:

A Solid - liquid waste systems amnagement

B Modelling of solid - liquid systems (CFD, DEM)

I NEED HELP with the following research gap(s) or need(s):

A

B

I CAN (most likely) HELP with the following research gap(s) or need(s):

A

B 
NAME: Mike Goff

ORGANISATION/INSTITUTION: Idaho National Laboratory

MY (or my institution's) MAIN AREA(S) OF RESEARCH EXPERTISE:

A Non aqueous processing technology pyro processing

B

I NEED HELP with the following research gap(s) or need(s):

A

B

I CAN (most likely) HELP with the following research gap(s) or need(s):

A Capabilities from bench to pilot scale far pyno processing of metal fuel currently fast reactor fuels with sodium boading

B 
NAME: Kostedin Ivanov / Sevnggin Kim PI

ORGANISATION/INSTITUTION: Penn State University

MY (or my institution's) MAIN AREA(S) OF RESEARCH EXPERTISE:

A Modelling and code benchmaking of neutronic / TH codes

B

I NEED HELP with the following research gap(s) or need(s):

A

B

I CAN (most likely) HELP with the following research gap(s) or need(s):

A Modelling capability (fundamentally bases) of non-designed based accidents and transients

B 
NAME: Andy Klein

ORGANISATION/INSTITUTION: Oregon State University

MY (or my institution's) MAIN AREA(S) OF RESEARCH EXPERTISE:

A Crud modelling and fuel performance simulation POC: Andy Klein

B Fuel performance codes

I NEED HELP with the following research gap(s) or need(s):

A Crud model development/data evalulation

B

I CAN (most likely) HELP with the following research gap(s) or need(s):

A

B 
NAME: T Toteu

ORGANISATION/INSTITUTION: ANL

MY (or my institution's) MAIN AREA(S) OF RESEARCH EXPERTISE:

A Development of probabilistic safety assessment models

B

I NEED HELP with the following research gap(s) or need(s):

A

B

I CAN (most likely) HELP with the following research gap(s) or need(s):

A Development of probabilistic thermal - hydraulics and safety analysis models

B 
NAME: Ian Farnan POC/PI

ORGANISATION/INSTITUTION: University of Cambridge Earth Sciences

MY (or my institution's) MAIN AREA(S) OF RESEARCH EXPERTISE:

A Local structure in complex materials - kadiation damage in waste forms and fuels

B Aqueous durability waste forms - combination of $A$ and $B$

I NEED HELP with the following research gap(s) or need(s):

A Access to radiological samples prep \& characterisation facilities

B propagation into higher scale models (of atonistic/mechanistic date) using thermodynamic/reactive transport techniques - uncertainty quantification methods

I CAN (most likely) HELP with the following research gap(s) or need(s):

A Element specific spectroscopy of waste forms and fuels $U$ and Th only (non irradiated)

B Quantification of radiation damage calibration of models / MD simulations (protonation). Quantification of waste form and leautate in aqueous durability expts 
NAME: Andy Klein

ORGANISATION/INSTITUTION: Oregon State University

MY (or my institution's) MAIN AREA(S) OF RESEARCH EXPERTISE:

A radio chemistry and chemical separations $\mathrm{POC}$ : Alena Pavlenova

B

I NEED HELP with the following research gap(s) or need(s):

A

B

I CAN (most likely) HELP with the following research gap(s) or need(s):

A Chemical separations of small quantities of activides

B 
NAME: Ram Devanathan

ORGANISATION/INSTITUTION: PNNL

MY (or my institution's) MAIN AREA(S) OF RESEARCH EXPERTISE:

A Modelling of phase development in irradiated fuel

B Modelling radiation damage of fuel

I NEED HELP with the following research gap(s) or need(s):

A Linking atomistic studies to finite element models

B

I CAN (most likely) HELP with the following research gap(s) or need(s):

A Phase development in irradiated fuel POC: Shenyang Hu Shenyang.hu@pnl.gov

B Modelling radiation damage of fuels POC: ram-devanathan@pnl.gov 
NAME: Jason Hayward (jhayward@utk.edu) Wes Hines jhinesz@utk.edu

ORGANISATION/INSTITUTION: University of Tennessee Oak Ridge National Lab

MY (or my institution's) MAIN AREA(S) OF RESEARCH EXPERTISE:

A Reactor design, ops, monitoring

B

I NEED HELP with the following research gap(s) or need(s):

A

B

I CAN (most likely) HELP with the following research gap(s) or need(s):

A Data validation, online monitoring of disjoints (Hines) data, and nuclear materials diversion in nhc facilities

B Real time in-core sensors for high temp, high flux environments (Hayward) 
NAME: Graham Fairhall

ORGANISATION/INSTITUTION: UK NNL

MY (or my institution's) MAIN AREA(S) OF RESEARCH EXPERTISE:

A Waste Immobilisation

B irradiation metal fuel behaviour

I NEED HELP with the following research gap(s) or need(s):

A performance / corrosion of $U$ fuel in immobilisation matrix

B U Species in high $\mathrm{PH}$ environment

I CAN (most likely) HELP with the following research gap(s) or need(s):

A Novel waste forms for reactive waste

B Spent fuel treatment / separation 
NAME: Ken Nash

ORGANISATION/INSTITUTION: Washington State University (Chemistry)

MY (or my institution's) MAIN AREA(S) OF RESEARCH EXPERTISE:

A Actinide solution Chemistry

B Aqueous separation / fuel ruprocessing

I NEED HELP with the following research gap(s) or need(s):

A

B

I CAN (most likely) HELP with the following research gap(s) or need(s):

A Radio chemistry facilities (fnoces up to $\mathrm{mg}$ of trus)

B Advanced Fuel cycle (separations). Actinide solution chemistry Thermodynamic and kinetics 
NAME: POC Sandarstan K Loyalka (also covering for colleagues)

ORGANISATION/INSTITUTION: University of Mo Columbia

MY (or my institution's) MAIN AREA(S) OF RESEARCH EXPERTISE:

A Fuel fission gen transport and heat transent

B Multi physics / multi scale modelling

I NEED HELP with the following research gap(s) or need(s):

A If ( fuel technology: advances and alternative fuels: computation)

B Ic (fuel technology: fuel performance codes)

I CAN (most likely) HELP with the following research gap(s) or need(s):

A Advance computations

B 
NAME: Sherrell Greens POC

ORGANISATION/INSTITUTION: ORNL

MY (or my institution's) MAIN AREA(S) OF RESEARCH EXPERTISE:

A Nuc. Fuel performance. Multi scale /multi physics

B

I NEED HELP with the following research gap(s) or need(s):

A

B

I CAN (most likely) HELP with the following research gap(s) or need(s):

A NUC. Fuel performance modelling

B NUC. Fuel performance fuel testing 
NAME: Neil Hyatt

ORGANISATION/INSTITUTION: University of Sheffield

MY (or my institution's) MAIN AREA(S) OF RESEARCH EXPERTISE:

A Waste form design processing characterisation and performance evaluation - inc. glass ceramics, cement. Expertise in pu disposition im particule

B Acoustic emission spectroscopy applied to remote sensing of waste form evolution e.g. cracking, volume expansion

I NEED HELP with the following research gap(s) or need(s):

A Ion implantation techniques to simulate radiation damage in materials, computational modelling techniques applied to simulate radiation damage

B Advanced term and surface analysis techniques applied to study corroded waste form materials

I CAN (most likely) HELP with the following research gap(s) or need(s):

A Waste form expertise for source fenn input into transport modelling

B Samples for thermodynamics studies of elements incorparation in waste form 
NAME: Jerry Fairley

ORGANISATION/INSTITUTION: University of Idaho

MY (or my institution's) MAIN AREA(S) OF RESEARCH EXPERTISE:

A Multiphase flow and heat transport modelling

B Site characterisation and geostatistical analysis of field data for heterogeneous sites

I NEED HELP with the following research gap(s) or need(s):

A Access to field sites (surface and underground)to test models and characterisation methods collaborators with expertise in chemistry for THC and THMC modelling of complex geological systems (thermal hydro chemical and thermal hydro mechanical chemical)

B

I CAN (most likely) HELP with the following research gap(s) or need(s):

A Numerical simulation, geo statistical analysis, uncertainty analysis

B Site characterisation program design and data collection / analysis. Hydrologic, thermal, and thermal - hydrologic modelling 
NAME: (Point of Contact) lan Hutchinson

ORGANISATION/INSTITUTION: MIT

MY (or my institution's) MAIN AREA(S) OF RESEARCH EXPERTISE:

A MIT reactor 5MW with fluxes comparable to LWR, internal loops for research testing

B

I NEED HELP with the following research gap(s) or need(s):

A

B

I CAN (most likely) HELP with the following research gap(s) or need(s):

A Coolant chemistry \& other future reactor research requiring in-core loops etc

B NUC. Materials \& fuel testing in small scale reactor (Gateway to TR research through prototyping) 
NAME: Robin Grimes

ORGANISATION/INSTITUTION: Imperial College London

MY (or my institution's) MAIN AREA(S) OF RESEARCH EXPERTISE:

A Phosphate waste forms for chloride and activite based waste forms

B The structures of glass - crystal interfaces and segregation of species to these interfaces

I NEED HELP with the following research gap(s) or need(s):

A Experimental thermodynamics of waste form behaviour

B Experimental verification of interface structures

I CAN (most likely) HELP with the following research gap(s) or need(s):

A Utilization of waste

B Transport modelling 
NAME: Greg Stormbury 208.526.1241

ORGANISATION/INSTITUTION: Idaho national Laboratory

MY (or my institution's) MAIN AREA(S) OF RESEARCH EXPERTISE:

A Multi phase modelling

B Site Characterisation

I NEED HELP with the following research gap(s) or need(s):

A

B

I CAN (most likely) HELP with the following research gap(s) or need(s):

A Numerical modelling of multi phase

B Site characterisation 
NAME: Per Peterson (Wirth, POC)

ORGANISATION/INSTITUTION: UN Berkeley Department of Nuclear Engineering

MY (or my institution's) MAIN AREA(S) OF RESEARCH EXPERTISE:

A Thermal hydraulics analysis of molten salt cooled reactors, including scaled \& integral experimentation

B

I NEED HELP with the following research gap(s) or need(s):

A

B

I CAN (most likely) HELP with the following research gap(s) or need(s):

A Development of high temperature reactor designs

B Development of optimal integral experiments 
NAME: Paul Howarth

ORGANISATION/INSTITUTION: UK NNL

MY (or my institution's) MAIN AREA(S) OF RESEARCH EXPERTISE:

A Fuel cycle analysis

B Modelling and simulation

I NEED HELP with the following research gap(s) or need(s):

A Standard nuclear data and integrated codes for fuel cycle assessment

B Flow sheet data

I CAN (most likely) HELP with the following research gap(s) or need(s):

A Access to UK codes and hisnris fuel cycle inventory database

B 
NAME: Tunc Aldemir (PITBI)

ORGANISATION/INSTITUTION: Ohio State University

MY (or my institution's) MAIN AREA(S) OF RESEARCH EXPERTISE:

A Modelling of Multi phase transport

B

I NEED HELP with the following research gap(s) or need(s):

A

B

I CAN (most likely) HELP with the following research gap(s) or need(s):

A Modelling of multi phase transport

B 
NAME: John Ireland - POC

ORGANISATION/INSTITUTION: Los AImos

MY (or my institution's) MAIN AREA(S) OF RESEARCH EXPERTISE:

A Reaction design ops \& mondring

B Thorism fuel cycles small LWR's

I NEED HELP with the following research gap(s) or need(s):

A

B

I CAN (most likely) HELP with the following research gap(s) or need(s):

A UQ \& V\&V - Cetin Unal (POC)

B 
NAME: Colin Boxall

ORGANISATION/INSTITUTION: Lancaster University

MY (or my institution's) MAIN AREA(S) OF RESEARCH EXPERTISE:

A Novel analytical methods and sensors. Novel membrane for separation and dialysis electro osmosis.

B Urex and novel reprocessing routes. Chemical / hydrodynamic kinetic modelling

I NEED HELP with the following research gap(s) or need(s):

A Thin film fabrication at $\mathrm{T}<500 \mathrm{c}$ (ceria, urania, titania)

B Calorimetry for water absorption at ceria etc surfaces

I CAN (most likely) HELP with the following research gap(s) or need(s):

A Chem reaction transport

B 
NAME: Joonhong Ahn / Wirth, POC

ORGANISATION/INSTITUTION: UC Berkeley, Department of Nuclear Engineering

MY (or my institution's) MAIN AREA(S) OF RESEARCH EXPERTISE:

A Computational modelling and analysis of geologic repository performance

B Multi physics simulations of radio nuclick transport in geologic settings

I NEED HELP with the following research gap(s) or need(s):

A

B

I CAN (most likely) HELP with the following research gap(s) or need(s):

A Numerical modelling of multi phase transport at multi scales in highly heterogeneous porons materials

B 
NAME: Kostadin Ivanov

ORGANISATION/INSTITUTION: Penn State University

MY (or my institution's) MAIN AREA(S) OF RESEARCH EXPERTISE:

A Generic maths approaches to multiscale modelling

B Coupled codes for neutronic/thermal hydraulic. Benchmarking of codes

I NEED HELP with the following research gap(s) or need(s):

A

B

I CAN (most likely) HELP with the following research gap(s) or need(s):

A See above in expertise

B 
NAME: George Smith

ORGANISATION/INSTITUTION: Oxford University

MY (or my institution's) MAIN AREA(S) OF RESEARCH EXPERTISE:

A Materials Characterisation

B

I NEED HELP with the following research gap(s) or need(s):

A"Harvested " samples

B

I CAN (most likely) HELP with the following research gap(s) or need(s):

A Atomic - scale micro structural and compositional changes quantitive measurements

B 
NAME: Ken Nash (Contact) Nathalie Wall (PI)

ORGANISATION/INSTITUTION: Washington State University (Chemistry)

MY (or my institution's) MAIN AREA(S) OF RESEARCH EXPERTISE:

A Environmental actinide Chemistry

B

I NEED HELP with the following research gap(s) or need(s):

A

B

I CAN (most likely) HELP with the following research gap(s) or need(s):

A Inert atmosphere capability to mimic Rep

B Radiological lab facilities 


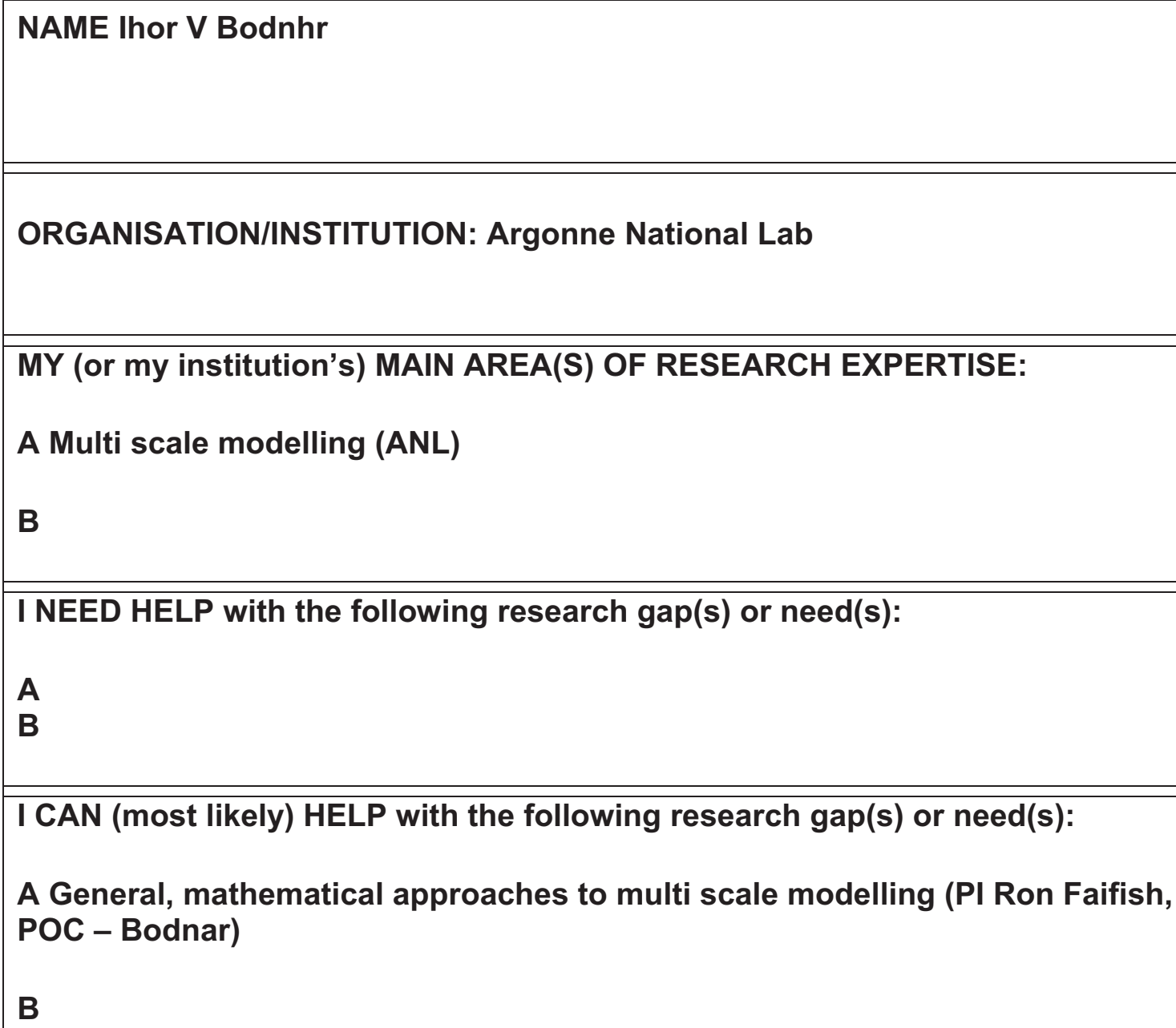


NAME: Graham Fairhall

ORGANISATION/INSTITUTION: UK NNL

MY (or my institution's) MAIN AREA(S) OF RESEARCH EXPERTISE:

A Steel corrosion in high radiation environment in bow $\mathrm{PH}$

B Chemistry of high level waste

I NEED HELP with the following research gap(s) or need(s):

A Radioactive species in HLW that effect corrosion

B Ability total representative samples from with radiation plants

I CAN (most likely) HELP with the following research gap(s) or need(s):

A Aging of complex HLW - physical/chemical properties

B Modelling of heat generating tank storage problems 
NAME: Audeen Fentiman

ORGANISATION/INSTITUTION: Purdue University

MY (or my institution's) MAIN AREA(S) OF RESEARCH EXPERTISE:

A Environmental waste management

B Storage and disposal

I NEED HELP with the following research gap(s) or need(s):

A

B

I CAN (most likely) HELP with the following research gap(s) or need(s):

A Skills, training and education

B public understanding 


\section{NAME Mark Anderson/ Mike Corradini}

ORGANISATION/INSTITUTION: Wisconsin

MY (or my institution's) MAIN AREA(S) OF RESEARCH EXPERTISE:

A Heat transfer / fluid flow

B

I NEED HELP with the following research gap(s) or need(s):

A

B

I CAN (most likely) HELP with the following research gap(s) or need(s):

A Loop testing (water, co2, sodium, molten salt)

B 
NAME: Martin Newby PITBI

ORGANISATION/INSTITUTION: City University London

MY (or my institution's) MAIN AREA(S) OF RESEARCH EXPERTISE:

A Stochastic modelling: aging and degradation processes in life cycle models from concept to end of life

B Decision modelling for process management. Uncertainty elicitation (subjective) and uncertainty propagation

I NEED HELP with the following research gap(s) or need(s):

A

B

I CAN (most likely) HELP with the following research gap(s) or need(s):

A System and process monitoring linking anitical characteristics to operational and management decisions PRA

B Uncertainty quantification and management 
NAME: Jason Hayward (haywardjp@ornl.gov)

ORGANISATION/INSTITUTION: Oak Ridge National Lab University of Tennessee

MY (or my institution's) MAIN AREA(S) OF RESEARCH EXPERTISE:

A SNF and waste management

B

I NEED HELP with the following research gap(s) or need(s):

A

B

I CAN (most likely) HELP with the following research gap(s) or need(s):

A Facilities for safeguards related characterisation studies (coupled end to end demonstration) solid / aqueous form

B 


\section{NAME Tunc Aldemir (POC)}

ORGANISATION/INSTITUTION: Ohio State University

MY (or my institution's) MAIN AREA(S) OF RESEARCH EXPERTISE:

A Probabilistic risk assessment, uncertainty propagation in non-linear and systems

B Instrumentation /untrol

I NEED HELP with the following research gap(s) or need(s):

A Financial risk modelling

B

I CAN (most likely) HELP with the following research gap(s) or need(s):

A Dealing with debelesthie in I \& C Systems

B Wireless technologies. Propagation of risk in long term. UQ for all surries of uncertainties 
NAME: Marcelo Sanchez

ORGANISATION/INSTITUTION: University of Strathclyde

MY (or my institution's) MAIN AREA(S) OF RESEARCH EXPERTISE:

A Modelling - thermo - hydro - mechanical - chemical models for design of backfill materials and bentonite seals

B

I NEED HELP with the following research gap(s) or need(s):

A

B

I CAN (most likely) HELP with the following research gap(s) or need(s):

A backfill design and THMC modelling of days

B 